University of Tennessee Health Science Center

UTHSC Digital Commons

\title{
The Multilevel, Multicultural, and Multi-Temporal Ecosocial Framework of Population Health : How Neighborhoods, Culture, and History Impact Health Outcomes and Produce Health Disparities
}

Lawrence Tarves Brown

University of Tennessee Health Science Center

Follow this and additional works at: https://dc.uthsc.edu/dissertations

Part of the Health and Medical Administration Commons

\section{Recommended Citation}

Brown, Lawrence Tarves, "The Multilevel, Multicultural, and Multi-Temporal Ecosocial Framework of Population Health : How Neighborhoods, Culture, and History Impact Health Outcomes and Produce Health Disparities" (2010). Theses and Dissertations (ETD). Paper 33. http://dx.doi.org/10.21007/ etd.cghs.2010.0037. 


\title{
The Multilevel, Multicultural, and Multi-Temporal Ecosocial Framework of Population Health : How Neighborhoods, Culture, and History Impact Health Outcomes and Produce Health Disparities
}

\begin{abstract}
According to findings in the field of genetics, it has now been established that the impact of race on health is mostly a function of a people's lived experience, not their genetic make-up. Due to this, the role of race in the study of health outcomes and health disparities has traditionally been specified improperly in statistical models due to confounding with ethnicity. Additionally, the role of geography or the impact of neighborhoods on health is often not specified properly.

Thus, the author proposes a multilevel, multicultural, and multi-temporal ecosocial framework that explains the impact of neighborhoods on health and the role of race via ethnicity. This conceptual framework builds on the work of social scientists while advancing researchers' understanding of the role of neighborhoods, culture, history, and socially assigned race as it relates to health outcomes and the existence of health disparities.

Data collected from 5,314 participants in the Jackson Heart Study (in Jackson, Mississippi) were used to analyze and test the conceptual framework. The outcomes of interest are cardiovascular disease risk factors and hospitalization due to ambulatory care sensitive conditions are tested in multilevel models. Dependent variables included: total physical activity scores, hypertension status, diabetes status, hypercholesterolemia status, and hospitalizations due to ambulatory care sensitive conditions.
\end{abstract}

Among the study sample in a cross-sectional analysis, factors such as higher income, car ownership, and having a larger social network were statistically significant predictors of higher physical activity scores. In the prospective analysis of hypertension status, none of the variables designed to test the conceptual framework was statistically significant; however, social status is implicated via the statistical significance of college education in more advantaged neighborhoods. In the prospective analysis of diabetes status, historical factors (unfair medical treatment), cultural factors (church attendance), and neighborhood factors (fast food restaurant availability) were statistically significant predictors of diabetes. Having perceptions of serious neighborhood problems were statistically significant predictors of hypercholesterolemia. In the cross-sectional analysis of hospitalizations due to ambulatory care sensitive conditions, only increased age was associated with the dependent variable (likely due to small sample size).

Based on the dissertation research and findings across the literature, the multilevel, multicultural, and multi-temporal ecosocial framework shows promise and deserves further investigation. If the proposed ecosocial framework is refined and proven to be valid in future research, it can possibly help transform the practice and delivery of health care. Medical care can be modified to first assess and then develop an ethnically congruent treatment plan informed by an individual's social identity and their view of the world of health and health care. Policy makers and health care administrators can pass policies that address issues of quality, perceptions, trust, preferences, and health stewardship for individuals and populations. Finally, health care systems around the world can be transformed to provide ethnically responsive and relevant care that is locally flexible, persistently proactive, and able to use evidence-based interventions to eliminate health disparities.

\section{Document Type}

Dissertation 


\section{Degree Name}

Doctor of Philosophy (PhD)

\section{Program}

Health Outcomes and Policy Research

\section{Research Advisor}

Shelley White-Means, Ph.D.

\section{Keywords}

Health disparities Jackson Heart Study Cardiovascular disease African Americans M3 Ecosocial Framework

\section{Subject Categories}

Health and Medical Administration | Medicine and Health Sciences 
The Multilevel, Multicultural, and Multi-temporal Ecosocial Framework of Population Health: How Neighborhoods, Culture, and History Impact Health Outcomes and Produce Health Disparities

\author{
A Dissertation \\ Presented for \\ The Graduate Studies Council \\ The University of Tennessee \\ Health Science Center
}

\author{
In Partial Fulfillment \\ Of the Requirements for the Degree \\ Doctor of Philosophy \\ From The University of Tennessee
}

By

Lawrence Tarves Brown

December 2010 
Copyright $(92010$ by Lawrence Tarves Brown All rights reserved 


\section{DEDICATION}

This work is dedicated to the memory of my father, Lawrence Robert Brown, and the entire Parker-Brown family. In addition, I dedicate this work to my ancestors for their sacrifice and perseverance, knowing that this day would come. Finally, this dissertation is in honor of two of my greatest heroes: Harriet Tubman and William Edward Burghardt Du Bois. 


\section{ACKNOWLEDGEMENTS}

I gratefully acknowledge the Creator of Life and all things for my life and health. I am also thankful to the Creator for providing me with P-Funk, New Orleans brass bands, conceptual soul, urban jazz, gospel music, and southern hip hop that functioned as the soundtrack to my writing.

In addition, I acknowledge my family for their support and unsurpassed belief in me. To my mother, Sharon Parker, who reared me and raised me, who read to me and nurtured me, giving me a love for learning. I am eternally grateful. You trained me to live in accordance with godly principles and to stand for that which I believe.

I am thankful for my brothers and sisters (Jerrel, Atara, Tenesha, and Isaiah) for your steadfast love and financial support when resources were scarce. To my nieces and nephews (Talia, Jaylianna, Simeon, Jeremiah, Parker, and Brenton), I am grateful for the fun we shared whenever I visited Tomball. I want this to be proof of what you can do and an illustration of the potential that lies inside of each of you. To my family in Texas: Aunt Ivy, Uncle Jessie, Aunt Barbara, and cousins Tracy, Rhonda, Tonya, and Gwen. Thank you all for your love and encouragement.

To my grandparents, Rev. Dr. S. J. Parker and Aressa Parker, thank you both for your encouragement and love. You have both been a source of inspiration and joy. You have taught me that learning knows no age limit and imagination knows no bound. You provided me with shelter at the beginning of this journey. You also supported me in my community activism, and for that, I am forever proud to call myself your grandchild.

To my community mentor, brother Shabaka Afrika, you have been a source of dialogue, strength, and feedback. I pray that this work provides insights to the future work that must be done. Thank you for letting me know when to focus on my dissertation. Thanks to you and brother James Deke Pope for listening to me practice for my dissertation defense. I also thank Adama Evans for the physical training and nutrition guidance that shall serve me for the rest of my days.

I also thank my colleagues at LeMoyne Owen College who gave me the opportunity to serve on a community-based participatory research grant: Dr. Cheryl Golden, Dr. Calverta McMorris, Dr. Ernestine Small, and Dr. Barbara Frankle. I appreciate the partnership we created and your support. I also acknowledge my good friend Troylyn Braud for your friendship and the laughter we shared. You were wonderful company in the midst of my journey and a companion in times of struggle and frustration.

To my academic mentors and committee members (Drs. White-Means, Hong, Mele, Taff, and Ozdenerol), thank you for your belief in me; and for working with me as I found my way. Thank you for not discouraging me, for having an encouraging word, for useful feedback, and helping sharpen this work. Dr. Mele, thank you for helping me to frame and conceptualize Figure 5 and for your great suggestion to improve Figure 6. I 
also thank you Dr. White-Means, for your unwavering support throughout my $\mathrm{PhD}$ journey, for guiding this dissertation from start to finish, and for your suggestion leading to Figure 8. You have been a mother and a mentor-and for that I am grateful. I am indebted to Saint George Relyea at the University of Memphis for his invaluable assistance with the statistical portion of this work. I also thank Dr. Marcellus Barksdale for your faith in me and other faculty at Morehouse College and the University of Houston for my academic training.

I also owe a special thanks to Dr. Rita Geier for her passion to pursue justice on behalf of African Americans at the University of Tennessee. Dr. Geier, you are a shining champion in my eyes. The Geier Fellowship has been a great aid. The computer I used to compose this dissertation was purchased through the Geier Fellowship.

I am thankful for the wonderful support provided to me by my Morgan State University family. Dr. Kim Sydnor, I thank you for your hospitality and grace. You are an angel. You gave me the flexibility to finish my dissertation and gave me a chance to begin a new journey. I am also thankful to Ms. Barbara Inman, Dr. Ndidi AmutahHardrick, and Dr. Shalon Irving for their support, advice, and collegiality. Dr. Kenyatha Loftis, you have provided me with a shining example and gave me tips to help ease my journey-I thank you. I extend a hearty thanks to Ms. Shirley Hancock, as well, for her keen eye and editorial wisdom. Your comments and feedback helped elevate the quality of this manuscript immensely_thank you.

Finally, I acknowledge the Jackson Heart Study. The Jackson Heart Study (JHS) is a collaborative study supported by the National Institutes of Health and the National Center for Minority Health and Health Disparities (study ID numbers: 5001; N01 HC95170; N01 HC95171; NOI HC95172) in partnership with Jackson State University, Tougaloo College, and University of Mississippi Medical Center. I especially owe Dr. DeMarc Hickson a large measure of gratitude for working to provide great amounts of data and assistance with obtaining permission from the Jackson Heart Study. Dr. Hickson also provided key insights into neighborhood measures, fast food restaurant availability, and multilevel modeling. I am also grateful to the participants in the Jackson Heart Study. May this work help lead to improved quality of care and longer life for you and your posterity. 


\begin{abstract}
According to findings in the field of genetics, it has now been established that the impact of race on health is mostly a function of a people's lived experience, not their genetic make-up. Due to this, the role of race in the study of health outcomes and health disparities has traditionally been specified improperly in statistical models due to confounding with ethnicity. Additionally, the role of geography or the impact of neighborhoods on health is often not specified properly.

Thus, the author proposes a multilevel, multicultural, and multi-temporal ecosocial framework that explains the impact of neighborhoods on health and the role of race via ethnicity. This conceptual framework builds on the work of social scientists while advancing researchers' understanding of the role of neighborhoods, culture, history, and socially assigned race as it relates to health outcomes and the existence of health disparities.

Data collected from 5,314 participants in the Jackson Heart Study (in Jackson, Mississippi) were used to analyze and test the conceptual framework. The outcomes of interest are cardiovascular disease risk factors and hospitalization due to ambulatory care sensitive conditions are tested in multilevel models. Dependent variables included: total physical activity scores, hypertension status, diabetes status, hypercholesterolemia status, and hospitalizations due to ambulatory care sensitive conditions.
\end{abstract}

Among the study sample in a cross-sectional analysis, factors such as higher income, car ownership, and having a larger social network were statistically significant predictors of higher physical activity scores. In the prospective analysis of hypertension status, none of the variables designed to test the conceptual framework was statistically significant; however, social status is implicated via the statistical significance of college education in more advantaged neighborhoods. In the prospective analysis of diabetes status, historical factors (unfair medical treatment), cultural factors (church attendance), and neighborhood factors (fast food restaurant availability) were statistically significant predictors of diabetes. Having perceptions of serious neighborhood problems were statistically significant predictors of hypercholesterolemia. In the cross-sectional analysis of hospitalizations due to ambulatory care sensitive conditions, only increased age was associated with the dependent variable (likely due to small sample size).

Based on the dissertation research and findings across the literature, the multilevel, multicultural, and multi-temporal ecosocial framework shows promise and deserves further investigation. If the proposed ecosocial framework is refined and proven to be valid in future research, it can possibly help transform the practice and delivery of health care. Medical care can be modified to first assess and then develop an ethnically congruent treatment plan informed by an individual's social identity and their view of the world of health and health care. Policy makers and health care administrators can pass policies that address issues of quality, perceptions, trust, preferences, and health stewardship for individuals and populations. Finally, health care systems around the 
world can be transformed to provide ethnically responsive and relevant care that is locally flexible, persistently proactive, and able to use evidence-based interventions to eliminate health disparities. 


\section{TABLE OF CONTENTS}

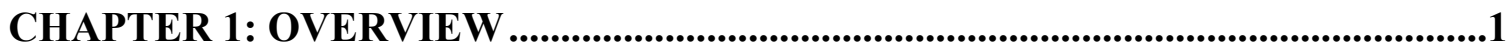

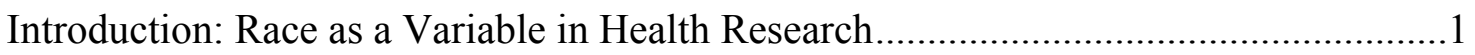

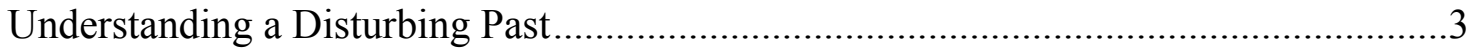

The Three Dominant Models of Medical Utilization.......................................................4

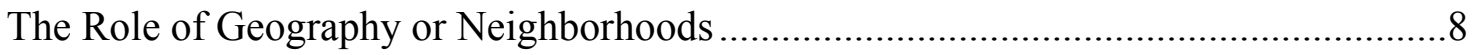

The Preventable Hospitalizations Problem ..............................................................

Why a New Conceptual Framework Is Needed ...........................................................

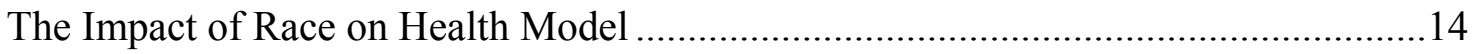

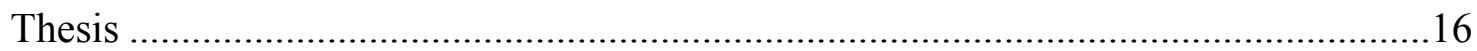

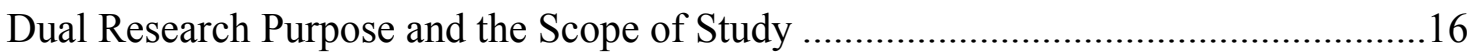

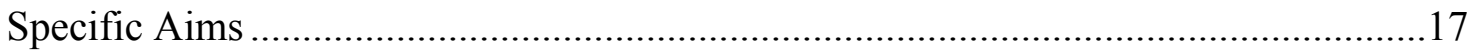

Overarching Framework and Key Definitions: Population Health and Health

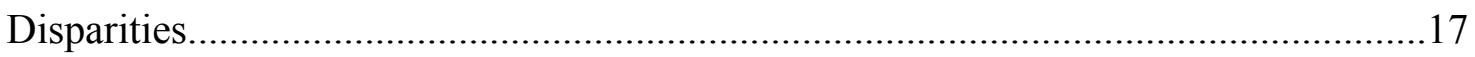

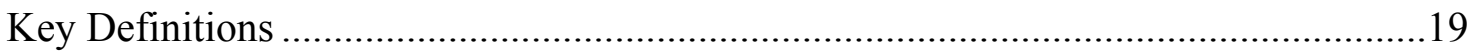

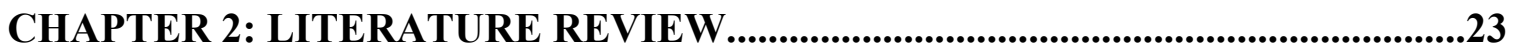

Part I: Disciplinary Explanations of Differences in Population Health ..........................23

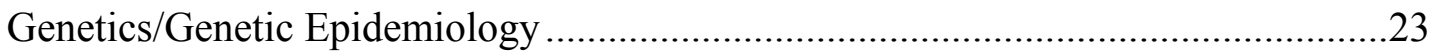

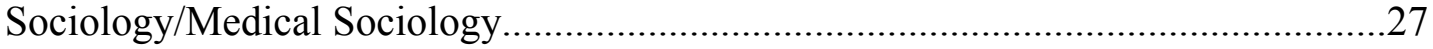

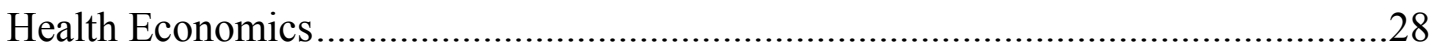

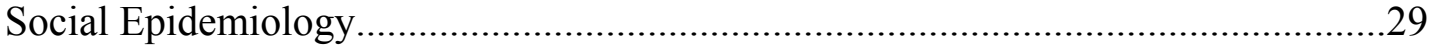

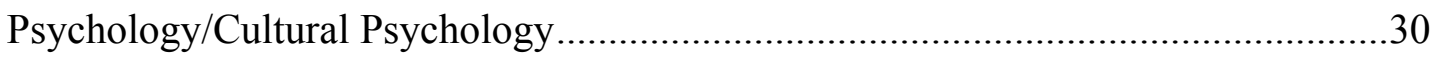

Religion or Psychology of Religion .....................................................................

Health Geography/Spatial Epidemiology ……………...............................................31

Health Services Research or Health Disparities Research .........................................32

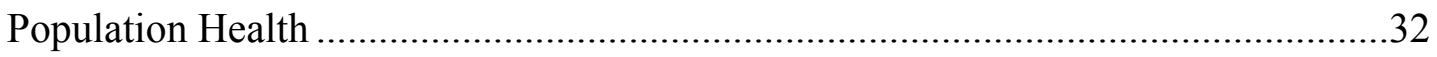

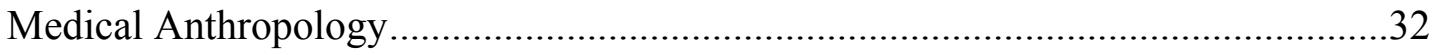

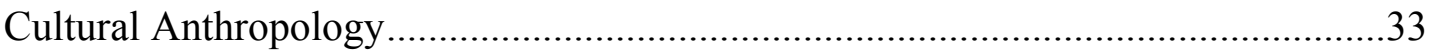

Part II: Review of Health Cosmology Literature .......................................................

Racial/Ethnic Differences in Health Stewardship .....................................................35

Racial/Ethnic Differences in Preference-Based Scores for Health States...................39

Racial/Ethnic Differences in Medical Trust ..............................................................44

Racial/Ethnic Differences in Perceptions of Provider or System Quality of Care.....49

Summary of the Literature Review on the Four Health Cosmology Factors ..............56

Part III: Review of Methodology Literature …………….........................................57

Review of the Use of Multilevel or Hierarchical Linear Modeling in Health

Literature .58 
Review of Geographic Information Science (GIS) Methodology in Health

Literature

CHAPTER 3: METHODOLOGY .61

Part I: The $\mathrm{M}^{3}$ Ecosocial Framework-An Ecosocial Theory .61

How Ethnicity Impacts Health Behaviors ............................................................61

Ethnicity and the Referential Aspect of Human Behavior .......................................63

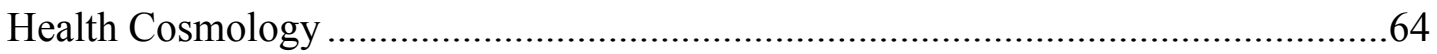

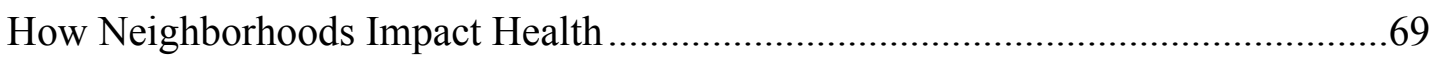

Historical Factors and Neighborhood Structure ................................................. 71

How History, Culture, and Neighborhood Factors Jointly Affect Individual

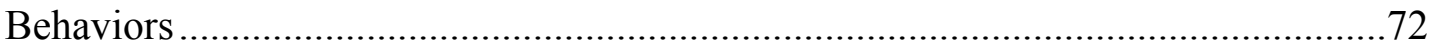

The $\mathrm{M}^{3}$ Ecosocial Framework in Light of Krieger's Ecosocial Theory ......................74

Part II: Quantitative Research Design .................................................................75

The Impact of Race on Health Model with the $\mathrm{M}^{3}$ Ecosocial Framework..................75

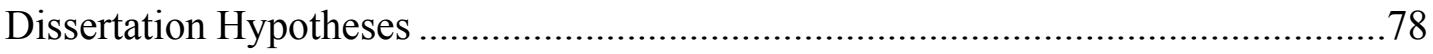

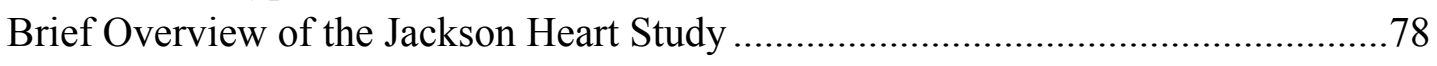

Recruitment and Sampling in the Jackson Heart Study ........................................... 78

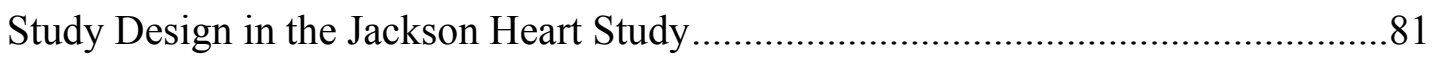

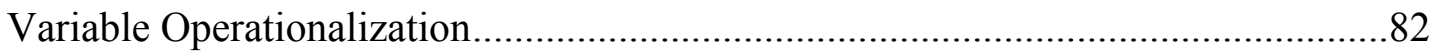

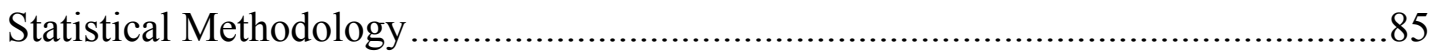

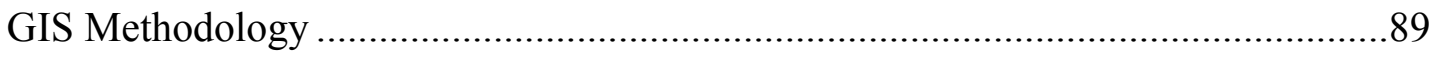

Specific Models for Each Dependent Variable ...................................................89

CHAPTER 4: RESULTS AND ANALYSIS..............................................................93

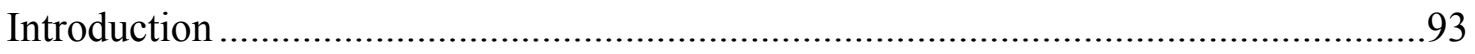

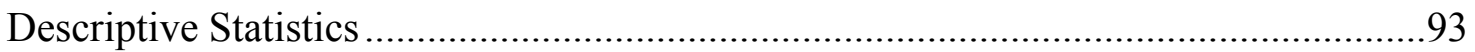

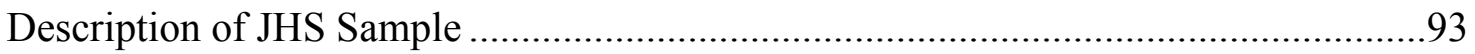

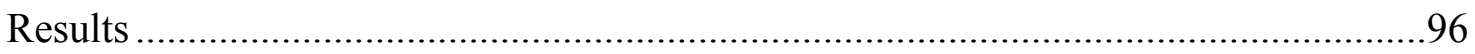

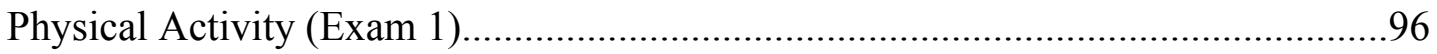

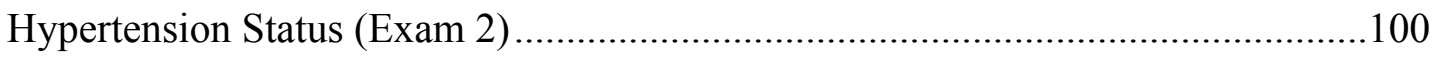

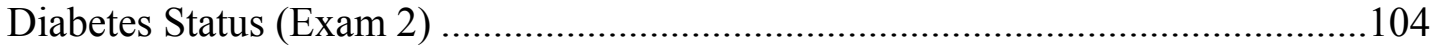

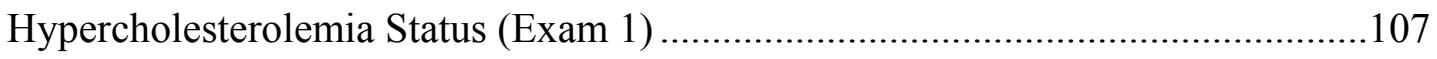

Hospitalization due to Ambulatory Care Sensitive Condition (ACSC) ...................110

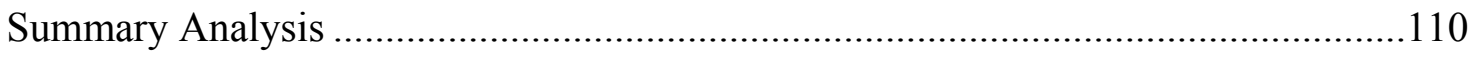

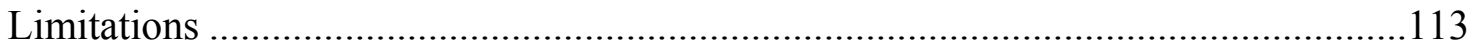

CHAPTER 5: DISCUSSION, IMPLICATIONS, AND TRANSFORMING THE HEALTH CARE SYSTEM IN THE UNITED STATES ...................................115

Research and Policy Implications of Dissertation Findings..................................115 
Research and Policy Implications of the $\mathrm{M}^{3}$ Ecosocial Framework ...........................117

Transforming the Health Care System in the United States......................................117

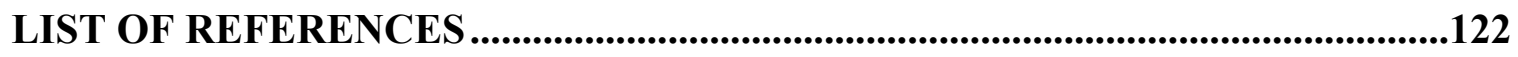

APPENDIX: JACKSON HEART STUDY CENSUS TRACTS..................................137

VITA 


\section{LIST OF TABLES}

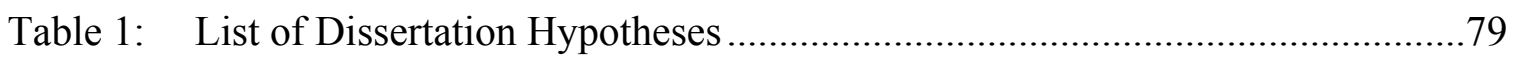

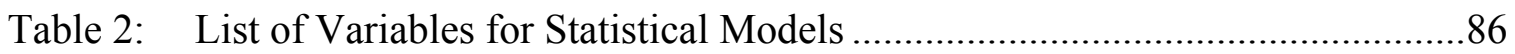

Table 3: Descriptive Statistics of the Jackson Heart Study............................................94

Table 4: Results for Physical Activity Score in Exam 1 in Overall Study Sample .......97

Table 5: Results for Physical Activity Score in Exam 1 in Least Advantaged

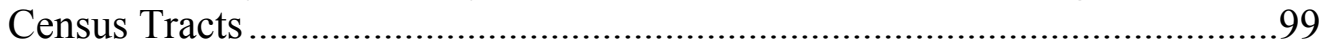

Table 6: Hypertension Status in Exam 2 in Overall Study Sample .............................101

Table 7: Hypertension Status in Exam 2 in Least Advantaged Census Tracts .............102

Table 8: Diabetes Status in Exam 2 in Overall Study Sample.....................................105

Table 9: Diabetes Status in Exam 2 in Least Advantaged Census Tracts.....................106

Table 10: Hypercholesterolemia Status in Exam 1 in Overall Study Sample.................108

Table 11: Hypercholesterolemia Status in Exam 1 in Least Advantaged Census

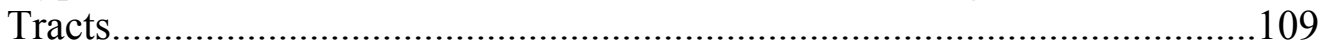

Table 12: ACSC Hospitalization from 2000-2009......................................................111

Table 13: List of Dissertation Hypotheses and Decision Rules ......................................112 


\section{LIST OF FIGURES}

Figure 1: Determinants of Health and Their Contribution to Premature Death ..............5

Figure 2: Differences, Disparities, and Discrimination: Populations with Equal

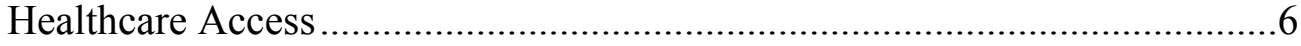

Figure 3: Ronald Andersen's Behavioral Model and Access to Medical Care (Phase

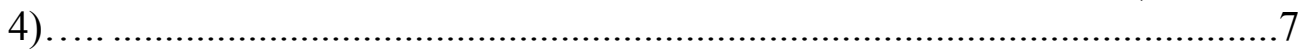

Figure 4: David R. Williams' Model of the Impact of Race on Health ........................15

Figure 5: How Culture and History Impact Health Outcomes and Produce Health Disparities

Figure 6: How Neighborhoods Impact Health Outcomes and Produce Health Disparities..................................................70

Figure 7: The Multilevel, Multicultural, and Multi-temporal $\left(\mathrm{M}^{3}\right)$ Ecosocial Framework ................................................... 73

Figure 8: The Impact of Race on Health Model with the $\mathrm{M}^{3}$ Ecosocial Framework ....76 


\section{CHAPTER 1: OVERVIEW}

\section{Introduction: Race as a Variable in Health Research}

Over 20 years ago, Wilkinson and King, two medical sociologists, published an article entitled "Conceptual and methodological issues in the use of race as a variable: Policy implications." In their article, Wilkinson and King (1987) highlighted many issues surrounding the way race is used as a variable in the social sciences. When race is used as an independent variable to help explain health outcomes, they argued (1987, p. 61),

...investigators rarely, if ever, clarify what meaning of race is being conveyed, what proportion of the variance in a given dependent variable, such as hypertension or survival rates from heart disease, can be accounted for by race as a biogenetic entity, as a social phenomenon, or what proportion can be explained in terms of the interaction between race and class.

A few years later, social psychologist James Jackson and colleagues joined the discussion concerning the way race is included in empirical or statistical models across the social sciences. Their research was summarized by White-Means (1995, p. 210) in the following manner:

$[\mathrm{R}]$ esearch indicates that race is a composite measure of social, psychological, biological, and genetic influences on a person's life. For example, affirmative action policies notwithstanding, there remain racial differences in labor market earnings opportunities. Likewise, educational attainment varies by race. A racial difference in genetic makeup is evidenced by differences in the effectiveness of medical interventions (primarily medications), according to race. The cultural norms of families, the experiences of discrimination, and the ability to cope with one's environment vary by race. Ethnicity and race are resources that form a context for behavior.

Economist Shelley White-Means (1995) focused on the way race as a variable was specified in empirical health economic models in her article entitled "Conceptualizing race in economic models of medical utilization: A case study of community-based elders and the emergency room." White-Means provided the first empirical evidence that cultural norms, apart from race as a genetic construct, play a role in explaining differences in medical utilization between Americans of European descent and African descent. She also advanced this critique by stating that it may be inappropriate to analyze the medical utilization of Americans of African descent and European descent without understanding important differences. White-Means (1995, p. 210) wrote:

Thus, race is a pervasive factor that interacts with many of the measures typically used to explain medical utilization patterns - socioeconomic status (income, education, health insurance coverage), attitudes, family cultures, and the incidence of disease. Given this type of interaction, each racial group should 
(from a methodological standpoint) be considered as a unique population whose behavior is affected by distinct social, psychological, and genetic environments.

Sociologists Gary King and David R. Williams (1995) echoed and extended the critique of race as a variable in health-related research. In the chapter entitled "Race and health: A multidimensional approach to African American health," King and Williams proposed a framework for understanding the relationship between race and health. Their model highlights a series of intervening factors (biological factors, cultural factors, socioeconomic factors, racism, and political factors) that in turn affect proximate factors (health practices, psychological stress, environmental stress, psychosocial resources, and medical care) that then alter biological processes to the point that disparities in health manifest between racial groups.

Concerning the use of the variable of race as it relates to health outcomes, King and Williams (1995, p. 101) wrote in the aforementioned chapter:

One reason why definitions of race are so inconsistent and unclear is that researchers employ the concept to measure every important indicator associated with racial inequality or difference. Presumably group socioeconomic status, cultural lifestyles and values, genetic predispositions and racism are all being measured by the race variable. Such a categorical or composite approach precludes independent analysis of the separate effects of each implied construct or determinant.

King and Williams also provide a full treatment on scholarly conceptions of the meaning of race and the ongoing debate surrounding the use of race in the social sciences. The Institute of Medicine (Smedley et al., 2003) also contributes to this discussion in a paper included in the Unequal Treatmen $t$ report entitled "Racial and ethnic disparities in healthcare: A background and history." Harawa and Ford (2009) extend this discussion by detailing the evolution of the scientific conception of race from the $17^{\text {th }}$ century to the modern day.

Many other researchers from a variety of disciplines have advanced critiques of the use of race in the scientific literature, including social scientists (William, 1997; Lin \& Kelsey, 2000; Jones, 2001; Ford \& Kelly, 2005; Pearson, 2008) and geneticists (Goodman, 2000; Pearce, 2004; Royal \& Dunston, 2004; The Race, Ethnicity, and Genetics Working Group, 2005). In spite of the richness of these critiques, many researchers continue to think uncritically of race as a variable. Most researchers do not make the critical distinction - to paraphrase Wilkinson and King-between race as a biogenetic entity and race as a social phenomenon. However, the time is ripe for a paradigm shift to occur. A clear picture is emerging in the literature from multiple disciplines: race is not biologically constructed via our genes but instead is socially constructed via behaviors defined by history, culture, and modes of identity (i.e., how we see ourselves and how we are seen by others). 


\section{Understanding a Disturbing Past}

In the United States, the historical context of race is complex, and many discussions are often politically charged. This context often makes it difficult to trace the contours of history, culture, and identity in order to show how race, or rather race relations, affects health outcomes. When considering the root causes of health disparities, the full context of race has not been incorporated into a cohesive framework that can explain why racial/ethnic groups such as African Americans underutilize medical care, or in a broader sense, can explain medical utilization patterns for populations. In other words, if researchers hope to truly understand medical utilization patterns in the United States and race as an explanatory variable, the history of race relations in the United States must be fully understood. As King and Williams (1995, p. 106) cogently explained:

An ahistorical approach - a failure to acknowledge and embody the past — can produce myopic and static theories and research... Moreover, on a larger scale, what results is a tremendous "historical vacuum" in the social sciences, which gives credence and opportunity for proponents of biological and genetic research to advance intergenerational theories or propositions about health phenomena based on heritability. Ahistorical approaches also enable simplistic and fragmented social theories and explanations (for instance, the culture of poverty) to gain currency and acceptability as intellectual paradigms and policy solutions.

To deal effectively with the persistence of health disparities and to formulate effective solutions, it is crucial that researchers understand the scope of the historical context in which health outcomes gestate and eventually emerge.

In Medical Apartheid, Harriet Washington (2006) detailed the disturbing history of abuses of African Americans at the hands of medical professionals in the United States from the period of enslavement to the present day. Essentially, Washington's work and others (King \& Williams, 1995; Toldson \& Toldson, 2001; Smedley et al., 2003; Gaskin et al., 2005; Randall, 2006; Pietila 2010) provides the crucial historical context for the existence of health disparities that continue to plague African Africans with shorter life expectancies, higher rates of morbidity, and higher rates of mortality when compared to other racial and ethnic groups. African American physicians also experienced challenges in training for the medical professions and endured bigotry, segregation, and exclusion (Baker et al. 2009; Washington et al., 2009).

With this historical context, it is clear that health disparities or differences in health outcomes between African Americans and European Americans have their origin and genesis in slavery, segregation, and discrimination - and were further compounded by discriminatory acts committed by European American doctors at the expense of African American patients. Additionally, contemporary experiences of racism have been shown to be damaging to health (Paradies, 2006; Mays et al., 2007). Given this context, it is clear that researchers should understand how racism impacts health and incorporate such an understanding into our theories, models, and conceptual frameworks that purport to explain variations in health behaviors, medical utilization, and health outcomes. 


\section{The Three Dominant Models of Medical Utilization}

The dominant models in health-related literature have yet to bring into focus the role of race or ethnicity in determining health behaviors, medical utilization, and health outcomes, especially hospitalization patterns for ambulatory care sensitive conditions (an intermediate health outcome) that often vary significantly by racial/ethnic groups. In Figure 1, Schroeder (2007) summarizes the literature on the determinants of health outcomes.

The model depicts what health researchers have found and stated throughout the literature: namely, that lifestyle/behaviors, genetics, physical and social environment, and medical care play a crucial role in determining population health. However, the roles of culture, history, and social identity are not specified.

In 2003, the Institute of Medicine (IOM) — led by Brian Smedley and others - released its seminal report entitled Unequal treatment: Confronting racial and ethnic disparities in health care. The report called for continued investigation into the study of the causal factors of health care disparities that once properly understood would lead to the elimination of disparities involving race and ethnicity. The report focused on patients and their clinical encounter in the healthca re setting to elucidate potential causes for health disparities.

After controlling for access to care, the IOM (Smedley et al., 2003, p. 4) stated that health differences are caused by: "1) clinical appropriateness and need or patient preferences, 2) the operation of healthcare systems and legal and regulatory climate, and 3) discrimination: bias, stereotyping, and uncertainty." According to the IOM, while health differences in the United States are caused by all three factors in the IOM model, health disparities are caused by only the last two factors (Figure 2). The first factor is viewed as a legitimate reason for observed differences in health outcomes, while the last two factors are viewed as causing unacceptable differences.

While the model developed by Gomes and McGuire (used by the IOM in its 2003 report) discussed various factors that may lead to differences in health outcomes, it does not explain why medical utilization patterns differ by racial or ethnic groups. In other words, this model depicts how disparities can occur within the health care system after patients access care. It does not describe how access to health care services may vary by racial or ethnic groups nor how race or ethnicity might play a role in determining how patients may seek care to deal with illnesses. It assumes equal access to health care by racial or ethnic group, and, by extension, an equal propensity among all racial or ethnic groups to seek health care services before they interact with the health care system. To be fair, this assumption was mandated, given the IOM's charge from Congress to study factors causing health disparities assuming equal access.

In the third model (Figure 3), Anderson depicts the variables involved in the use of health services and proposes pathways for how certain variables might impact the use of health services (1995). The environment (described by Andersen as physical, political, 


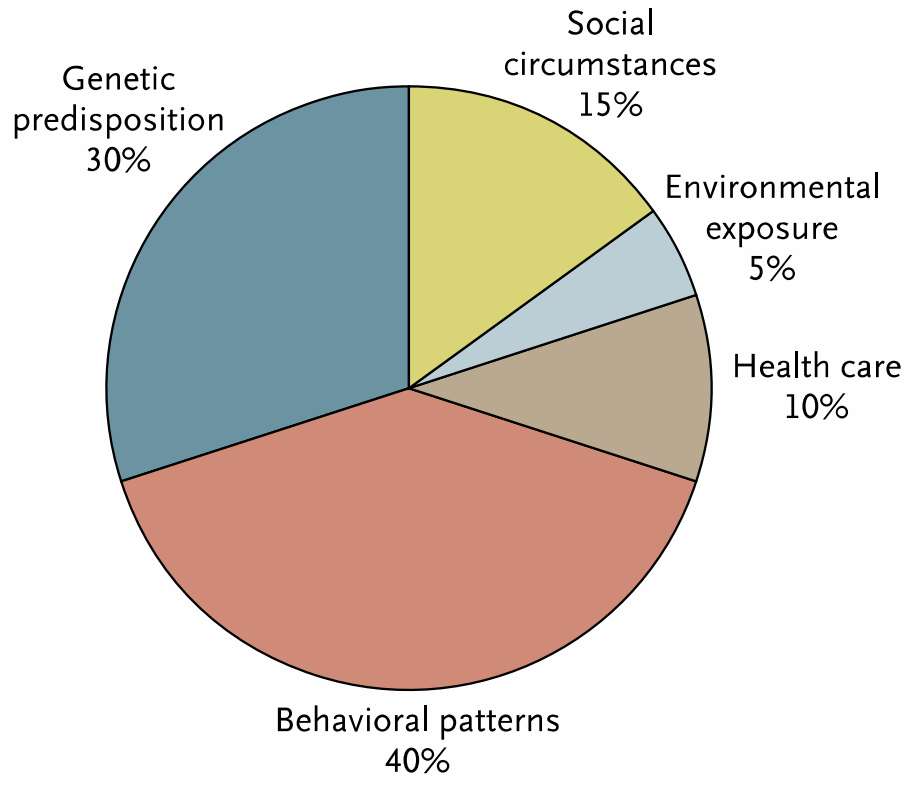

Figure 1: Determinants of Health and Their Contribution to Premature Death

Reprinted with permission. Schroeder, S. A. (2007). Shattuck lecture. We can do betterimproving the health of the American people. The New England Journal of Medicine , $357(12), 1221-1228$. 


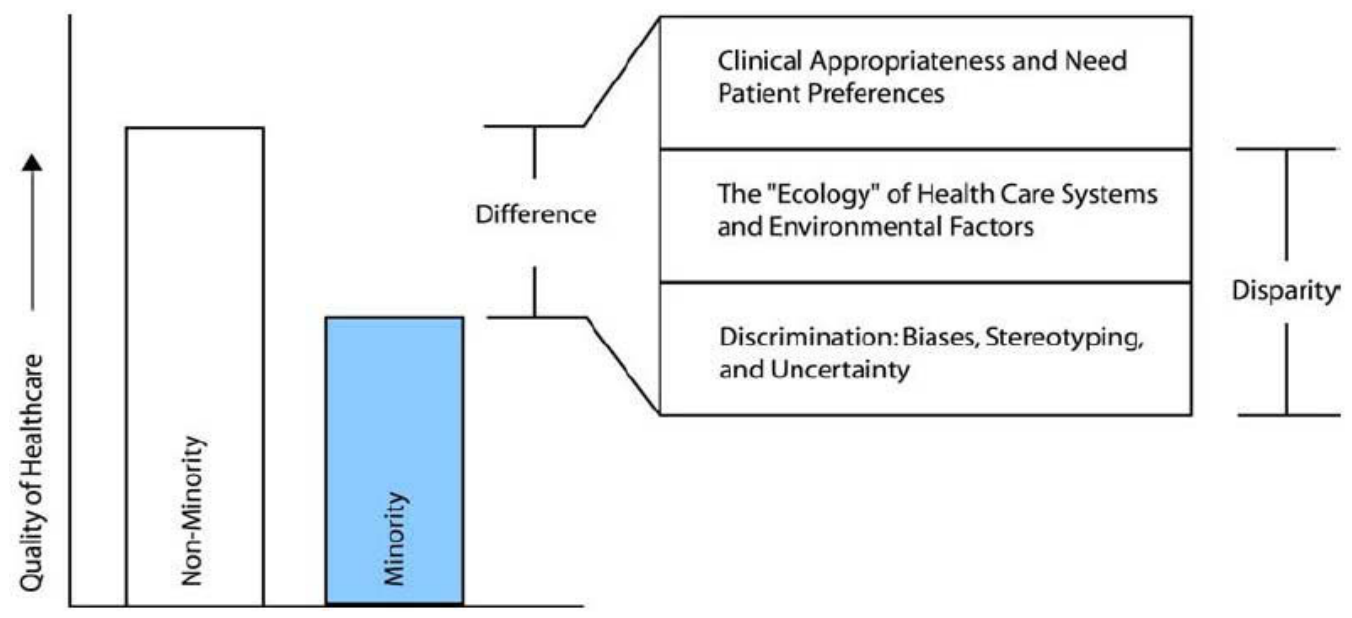

Figure 2: Differences, Disparities, and Discrimination: Populations with Equal Healthcare Access

Reprinted with permission. Smedley, B. D., Stith, A. Y., \& Nelson, A. R. (2003). Unequal Treatment: $C$ onfronting Racial and Ethnic Disparities in Health Care. Washington D.C.: National Academy Press. 


\section{$\begin{array}{llll}\text { ENVIRONMENT } & \text { POPULATION CHARACTERISTICS } & \text { HEALTH } & \text { BEHAVIOR } \\ & \text { OUTCOMES }\end{array}$}

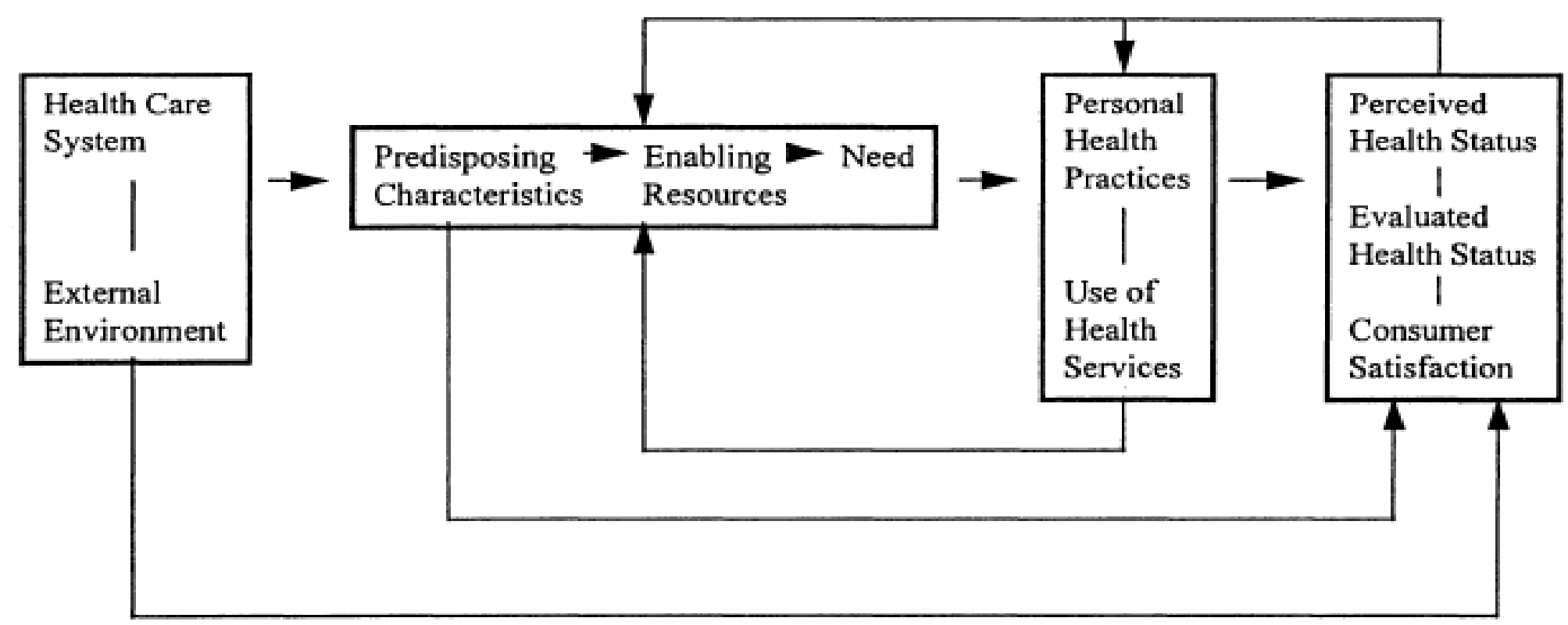

Figure 3: Ronald Andersen's Behavioral Model and Access to Medical Care (Phase 4)

Reprinted with permission. Andersen, R. M. (1995). Revisiting the behavioral model and access to medical care: Does it matter? Journal of Health and Social Behavior, 36(1), 1-10. 
and economic components) is shown as affecting the population via several characteristics, thereby partially determining patterns of health services utilization. In Andersen's model, the impact of race or ethnicity is not explicitly mentioned; nor is the role of race or ethnicity made clear.

\section{The Role of Geography or Neighborhoods}

Once we account for the fact that populations are nested inside geographic entities such as states, counties, or neighborhoods, we begin to see a more complete picture of how population health is affected and how health disparities are produced by the places in which humans live. In 2003, Ichiro Kawachi and Lisa Berkman published a book entitled Neighborhoods and Health where they weigh past evidence in the literature regarding how the places where people live impacts individuals' health above and beyond the influence of their individual characteristics. Kawachi and Berkman (2003, p. 24) noted that:

If we observe differences in health between places, these differences could be because of differences in the kinds of people who live in these places (a compositional explanation), or because of differences between places (a contextual explanation).... A mainly compositional explanation for geographical differences might tend to direct research and policy toward individuals, while a contextual explanation might direct attention toward health-damaging and health-promoting features of neighborhoods...

In other words, research into the connection between neighborhoods and health has farreaching implications for both research direction and policy formulation.

In Chapter 2 of Multicultural Medicine and Health Disparities, entitled "Geographic studies of black-white mortality," Levine and colleagues (2006) summarized the published literature in the field regarding mortality. Although Levine and colleagues examined the geographic variation for mortality, their findings might be expected to parallel future findings in studies involving geographic variation in hospitalization rates or morbidity in general. Levine and colleagues (2006, pp. 70-71) stated:

Taking factors associated with increased disparities in mortality as a composite, it would be expected they would comprise communities with high percentages of black residents, female-headed households, residential segregation, income inequality, and the presence of large housing projects. Residents of high-disparity communities would tend to have low levels of education, and would face both chronic unemployment and a disproportionate burden of cardiovascular disease, cancer, cirrhosis, and homicide.

Most of the studies reviewed by Levine and colleagues involved states or counties as the unit of analysis. In contrast, the field of neighborhoods and health examines small area variation or units of analysis such as zip codes, census tracts, and neighborhoods. In 
the field of neighborhoods and health, according to Altschuler et al. (2004), there have been four strands of research:

1) The contextual effects of neighborhoods

2) The impact of differential access to services and amenities (usually grocery stores and liquor stores)

3) The effect of a neighborhood's collective efficacy and social cohesion

4) The relationship between social capital and health

Research in this area shows that an individual's health outcomes are affected by where the individual lives based on varying features of their neighborhoods (Berkman \& Kawawchi, 2003; Altschuler, Somkin, \& Adler, 2004).

To summarize, research has shown that health is produced in a geographic context and that factors that affect health outcomes are, in essence, multilevel. But beyond the multilevel factors that affect health, there are other considerations that play a role in the structure of neighborhoods that are not adequately captured in the literature. Historical context is briefly discussed in the IOM report (2003, p. 103) as follows:

An historical account of the healthcare experience of African Americans is illustrative... of how the historic context shapes the contemporary structure of and access to care for racial and ethnic minorities. ...[T]he legacy of segregated and inferior healthcare for African Americans continues to reverberate in today's healthcare settings.

In other words, social policies and processes determine the structure of neighborhoods. The historical context determines which neighborhoods (and populations) have greater or lesser access to quality health care services (both in terms of provider supply and the number of health care facilities) and exposure to health-negating toxic environmental releases.

\section{The Preventable Hospitalizations Problem}

Thus, when the body of literature points to neighborhoods which are predominantly African American in composition as the areas containing the highest preventable hospitalization rates due to ambulatory care sensitive conditions (and contain the highest mortality rates due to these conditions), we must ask several questions. What is it about being identified as an African American that places this population at higher risk for disease and death? Is it due to genetic factors or is it due to ethnicity (i.e., due to referential, cultural, and historical factors)? If persons of African and European descent lived in equally impoverished neighborhoods (controlling for contextual explanation), would they have the same health outcomes? If not, why? 
The focus of this dissertation is on explaining racial/ethnic differences in health behaviors, medical utilization, and health outcomes - with a focus on the risk factors that lead to preventable hospitalizations or emergency room admissions due to ambulatory care sensitive conditions (Billings 2003). In his review of the empirical literature concerning preventable hospitalizations due to ambulatory care sensitive conditions (ACSC), Zahid Ansari (2007, p. 97) stated:

Racial differences have been observed across countries, with admission rates for ACSC higher among Blacks than Whites in the USA [and] among Aboriginal populations compared to non-Aboriginal in Australia, Maoris compared to nonMaoris in New Zealand, and Indian and Malays compared to Chinese in Singapore.

Thus, the issue of differences in preventable hospitalizations due to ambulatory care sensitive conditions is a global issue.

Ansari (2007) provided a list and description of the factors that have been tested in past research. As the list following demonstrates, the roles of social identity, culture, and history are not highlighted nor included as a determinant of preventable hospitalizations. The determinants of preventable hospitalizations due to ACSCs in the empirical literature include:

- Demographics (sex, age, race)

- Socioeconomic status

- Rurality

- Health system factors

- Prevalence of disease

- Lifestyle factors (smoking, physical activity, alcohol consumption, nutrition)

- Environment

- Adherence to medication

- Propensity to seek care

- Severity of illness

In their paper "Sociocultural methods in the Jackson Heart Study: Conceptual and descriptive overview," Payne and colleagues (2005, p. S6-38) stated: 
The role that sociocultural factors may play in explaining excess CVD risk and mortality in African Americans is poorly understood.... At a basic level, we have yet to ascertain why systematic differences exist in risk profiles across ethnic groups, and any differential manner in which [sociocultural factors] combine to determine ultimate outcomes.

Thus, we have arrived at a crossroads that for most researchers is an issue of semantics. Are differences we observe in health outcomes due to race or are those differences due to ethnicity? While the previous discussion on race as a variable in health research provides a sneak preview of the answer, this question also illuminates why a new conceptual framework is needed.

\section{Why a New Conceptual Framework Is Needed}

A new conceptual framework is needed because current theories poorly explain the role of race as a measure when included and tested in statistical models. We know that African Americans, indigenous Australians, Maoris, and the Indian and Malays in Singapore have worse health outcomes across a variety of measures than European Americans, non-indigenous Australians, non-indigenous New Zealanders, and Chinese populations in Singapore (Ansari, 2007). When examining health disparities from a global perspective, the genetic explanation for health disparities does not seem to make much sense, especially given the multiple global populations that bear a disproportionate burden of disease in different societies.

If the explanation for health disparities were rooted in genetic differences, then all of the global populations bearing a disproportionate burden of disease would possess genes in common that help produce poor health outcomes. Given the geographic distance between these populations, it is unlikely that inferior genes (as it relates to health) would develop in each of the respective populations. A more convincing explanation is the social construction of race, that is, how race is interpreted in each society. The interpretation of race is rooted in a population's culture and history and the meaning of socially assigned race (or social identity) within a given society. Without highlighting the roles of socially assigned race, culture, and history, health researchers are unable to clearly explain why certain populations across the planet have worse health in comparison to other comparable populations.

Simply put, the dominant models in health research and the empirical literature for preventable hospitalizations do not properly account for the fact that the measure of race is confounded with ethnicity —or a population's cultural background and historical experiences along with the social construction of race. This is no new revelation, as a large number of researchers have made exactly this point in the past. But what should be addressed, based on this body of literature, is that when we discuss race as a variable and determinant of health outcomes, it should be understood that the measure of race is confounded with four distinct phenomena: 1) genetic differences between populations, 2) differences in the way social hierarchies are arranged by race (historical experiences), 3) 
differences in the way populations behave (culture), and 4) social race assignment. The pivotal point is this: The variable of race in empirical models does more than just capture genetic differences among populations - culture, historical experiences, and social race assignment are all incorrectly nested in the categorization of race.

Therefore, the prevailing flaw in existing th eory and models is that roles of culture, history, an d socially assigned ra ce are not highlighted, and even when they are discussed, measures of culture, history, and social race assignment are not constructed nor tes ted collectively in empirical models. Thus, the roles of culture, history, and socially assigned race remain hidden. As we will see later in the definition of ethnicity (provided by William Dressler and colleagues), culture, history, and social race assignment are exclusive components of ethnicity, while genetic differences belong in the domain of biological phenomena such as DNA and phenotypes. This means wherever there is a discussion about behavior in the three dominant models used in health services and health disparities research - the Determinants of Health or Production Function of Health model (Figure 1 ), the Gomes and McGuire or IOM model (Figure 2 ), and Anderson and Aday's behavioral model (Figure 3 ) - what we are really discussing is ethnicity, because a person's ethnicity - their culture, historical experience, and socially assigned race-helps determine and predict their behaviors. In each model, 'behavioral patterns' (in Figure 1 ), 'patient preferences' (in Figure 2 ), and 'personal health practices' (in Figure 3), should be nested inside the ethnicity (the culture and historical experiences) of the individual or group in question.

Although the dominant models use different terms, the following terms are essentially different phrases for the same phenomena: 'behavioral patterns,' 'patient preferences,' 'personal health practices,' 'lifestyle,' or 'individual's behavior.' The only real difference might be contrasting 'patient preferences' with the other terms, but the difference lies in its use in the IOM model where the individual is in the health care environment. As such, 'patient preferences' is used to explain differences in medical decision-making by varying populations. This decision-making is essentially a behavior and therefore synonymous with the rest of the terms used in various models.

In terms of methodology, a new conceptual framework is also needed because most theories, models, and frameworks discussed and tested in the literature do not take into account the multilevel nature of populations and health. For instance, none of the dominant models discussed clearly show how individuals are affected by the neighborhoods in which they live. The McGinnis and colleagues' model does refer to "environmental exposure" in a very amorphous sense, while the Gomes and McGuire model examines health care system and environmental factors after the patient has accessed the health care system. The Anderson model does show health care system and external environment as determinants, but does not depict exactly how either environmental factor is purported to play a role in generating health outcomes. Finally, the Williams model (depicted in the next section) does not depict the impact of neighborhoods at all, although perhaps it touches on the impact of neighborhoods indirectly with "economic structures." In summary, these models lack a clear depiction of the role of neighborhoods on health. 
Without accounting for the fact that human populations are nested in neighborhoods, the past theories and their resulting analyses are reductionistic. By obscuring the impact of nesting, analytical results produce results that overestimate the impact of individual characteristics and underestimate the impact of the powerful neighborhood factors that shape the health behaviors, medical utilization, and health outcomes of individuals.

A new conceptual framework is also needed because our existing theories do not explicitly incorporate physical features of a neighborhood-or aspects of the built environment - that may have a great impact on population health. Although literature from sociology provides critical insight into the role of collective efficacy, social cohesion, and social capital as it relates to health outcomes, the availability and quality of health care facilities and the amount of toxic environmental releases every year in a neighborhood are more direct and specific measures of the effect of a neighborhood on a population's health. Kawachi and Berkman (2003, pp. 34-35) highlight this issue by saying:

One of the main problems in the study of neighborhoods and health is the lack of development of theories about plausible social, psychological, and biological links between specific features of the neighborhoods and specific health outcomes. ...If the study of neighborhoods and health is to move forward and contribute both to etiological understanding and policy formulation, it is crucial that we have better models and theories about how neighborhoods may influence health and that we use them to determine the appropriate area scale and type of area influence we wish to measure. Otherwise, we will be left with a legitimate "so what?" response to repeated demonstrations that there are neighborhood variations in a number of health indicators.

Altschuler and colleagues (2004, p. 1220) extend this critique by posing the following argument about social capital:

Though the social capital-health conceptualization has been utilized extensively in recent health research, the use of social capital as a concept has been criticized for a lack of conceptual clarity and obscuring fundamental relationships between health and the environment. For example, social capital has been used to describe civic and interpersonal trust, civic participation, social cohesion, and collective efficacy... The variability of its definition and use has been criticized because it has become a catch-all concept without distinct meaning or value....

They continue:

Muntaner and his colleagues have criticized the utilization of social capital on conceptual and political grounds.... They have argued that the use of social capital in public health research obscures the structural inequalities of class, race, and gender that are the main social factors that impact health. Pearce and Smith...recently advanced this argument, adding that social capital, among other 
variables, is a function of macro-level social and economic forces and can lead to "blame the victim" social policies (p. 1220).

The author agrees with the points raised by Altschuler and colleagues. The problem here is a matter of misspecification due to a lack of proper conceptualization. The proximity to and quality of health care facilities, grocery stores or food facilities, parks and fitness facilities, and toxic environmental release facilities as variables in studies involving neighborhoods and health are better variables that strengthen our ability to make more plausible links between specific features of neighborhoods and specific health outcomes. Health care facility availability, fresh fruit and vegetable availability, exercise facility/park/playground availability, and the amount of toxic environmental releases per neighborhood are not just measures that capture health-enhancing or health-negating facilities, they also capture the neighborhood's capacity to address or avoid health issues for the population within the neighborhood. In other words, these specific features of neighborhoods would be better ways to measure a population's social capital as it relates to resources they can draw upon to address health issues.

A new conceptual framework is needed that accounts for the multilevel nature of human populations by calling for the consistent acknowledgement of the impact of nesting in neighborhoods. Statistically, this means that multilevel or hierarchical linear modeling must be used to capture the effects of not only individual characteristics, but also neighborhood characteristics such as those mentioned previously.

Finally, a new conceptual framework is needed because policy is dependent upon research findings. In spite of policies that were designed to reduce health disparities in the United States, health disparities still exist with no end in sight. In a recent issue of the Journal of the American Medical Association , Rebecca Voelker (2008) reported that decades of work to reduce health disparities in the US had produced only limited success. Why have past efforts and policies not closed the gaps we observe in health outcomes? In order for policy to be effective, it must be based on comprehensive analysis. No solution can be effective if it is not based on a comprehensive understanding of the problem. But comprehensive analysis cannot take place without a clear and comprehensive conceptual framework to guide research and to help interpret findings.

\section{The Impact of Race on Health Model}

The model first developed by Gary King and David Williams, but later refined by David Williams (1997), represents the most advanced model of how race-in each of its multifaceted aspects - impacts health outcomes (Figure 4 ). The model developed by Williams illustrates how culture, biological \& geographic origins, racism, economic structures, and political and legal actions each impact health status from a macrosocial perspective.

However, the model developed by Williams does not illustrate how persons are nested within neighborhoods, nor does it illustrate how health practices are determined by an 


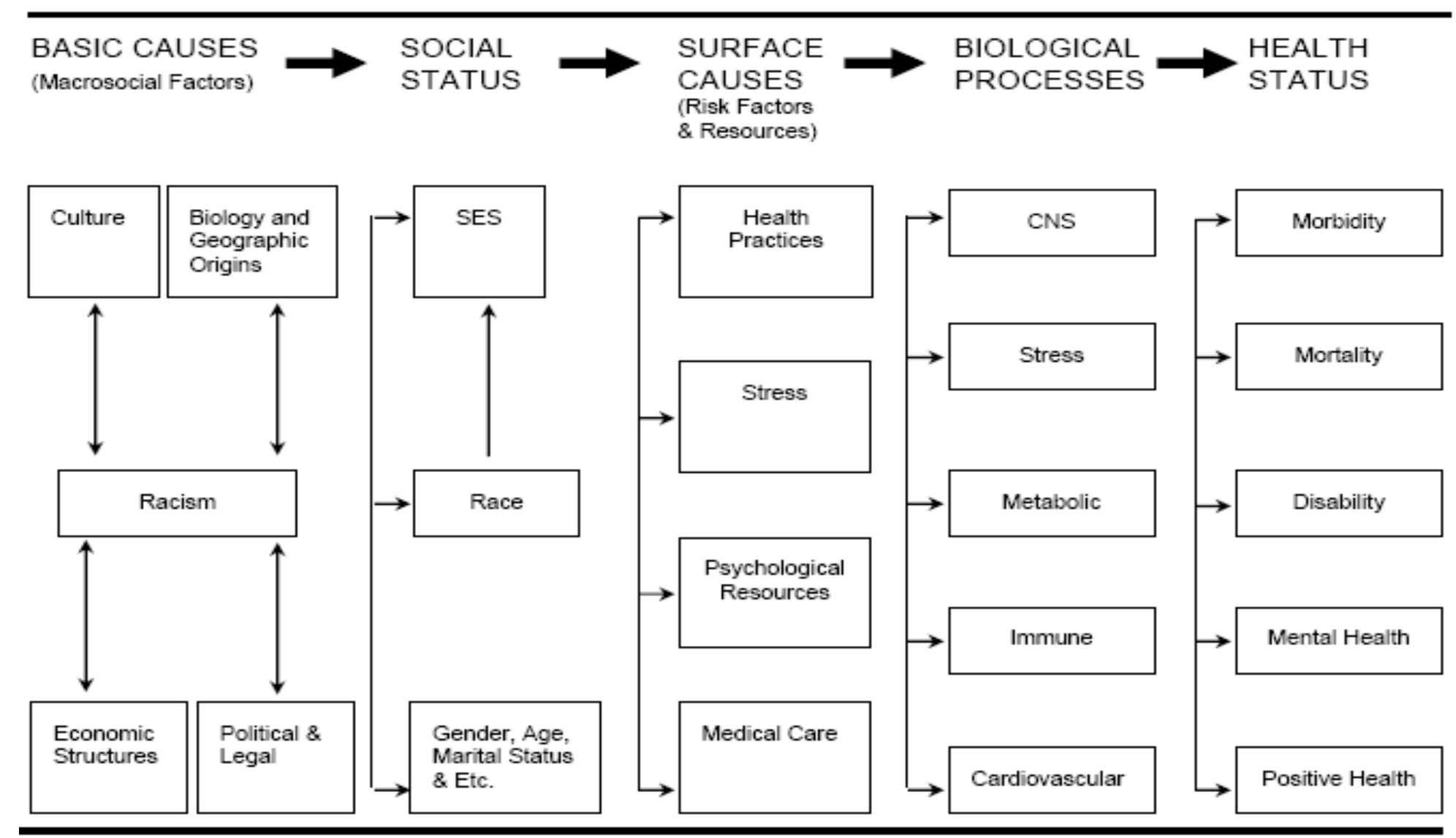

Figure 4: David R. Williams' Model of the Impact of Race on Health

Reprinted from Annals of Epidemiology, Volume 7, Issue 5, David R. Williams. Race and health: Basic questions, emerging directions, pp. 322-33, Copyright (1997), with permission from Elsevier. 
individual's social identity, culture, or historical experiences. In spite of this, the Williams model is an excellent starting frame of reference for structuring the analysis in this dissertation. The new ecosocial conceptual framework, developed and discussed in Chapter 3, complements Williams' model by showing the impact of ethnicity and neighborhoods from a microsocial perspective.

\section{Thesis}

It is the author's contention that the factors that affect health are not only multilevel, but also multicultural and multi-temporal. Individuals belong to various ethnic groups possessing different definitions of self, cultures, and historical experiences. Individuals are also situated or nested within neighborhoods. Neighborhoods are dynamic entities that are shaped by the residing populations' cultures and historical experiences. Neighborhoods are essentially a mirror that reflects the status of its residents within the society. In other words, neighborhoods with higher percentages of advantaged people are less likely to have high amounts of toxic environmental releases, while neighborhoods with higher percentages of disadvantaged people are more likely to have high amounts of toxic environmental releases. Once we properly elucidate how populations and neighborhoods are situated within a cultural and historical context and are affected by socially assigned race, we can then begin to create measures to capture the influence of culture, history, and socially assigned race to explain health outcomes and the existence of health disparities.

Although the focus in this dissertation is on differences between people of European and African descent in the United States, the discussion and analysis presented can be extended to any society where there are differences in health outcomes (New Zealanders of European descent vs. the Maori, Australians of European descent vs. the indigenous people of Australia, South Africans of European descent vs. indigenous South Africans, people in higher castes in India vs. people in lower castes in India, etc.).

\section{Dual Research Purpose and the Scope of Study}

Whereas Patton (2002, p. 215) explained that purpose of basic research is "to understand and explain" and basic researchers are interested in "formulating and testing theoretical constructs and propositions that ideally generalize across time and space," applied research "contribute[s] knowledge that will help people understand the nature of a problem in order to intervene, thereby allowing human beings to more effectively control their environment" (2002, p. 217). Particularly of note is that the field in which this dissertation is written is interdisciplinary-Health Outcomes and Policy Research. And as Patton (2002, p. 217) stated: "Societal concerns have given rise to a variety of new fields that are interdisciplinary in nature. These emerging fields reflect the longstanding criticism by policymakers that universities have departments but society has

problems" (emphasis mine). The broad problem at the heart of this dissertation is health disparities observed across the world among various populations. The specific problem 
at the heart of this dissertation is the existence of health disparities in the United States, which lead to increased rates of preventable hospitalizations due to ambulatory care sensitive conditions and result in early mortality for African Americans.

An interdisciplinary approach was used to achieve the aforementioned traditional and applied research objectives. Research from genetics to medical anthropology to psychology was synthesized in a quest to understand the roles of neighborhoods, socially assigned race, culture, and history in determining health outcomes. Additionally, multiple quantitative methodological approaches were employed. Hierarchical linear modeling and geographic information science methodologies were utilized to answer the research question. The use of multiple quantitative methodologies addressed both traditional and applied research objectives. In other words, this dissertation aimed to enrich and inform the study of health disparities by focusing on the social problems that impact health outcomes and produce health disparities.

\section{Specific Aims}

There were three specific aims in this research.

- Aim 1: Develop a new conceptual framework that explains how neighborhoods, culture, and history impact health outcomes and produce health disparities.

- Aim 2: Use multilevel quantitative techniques (multilevel modeling and geographic information systems) to explore pathways by which neighborhoods, culture, and history postulated in the new conceptual framework affect cardiovascular risk factors (diabetes, hypertension, and hypercholesterolemia) and cardiovascular disease outcomes (hospitalization due to ambulatory care sensitive conditions) in the Jackson Heart Study.

- Aim 3: Formulate policy recommendations based on the new conceptual framework and results from the concurrent mixed method analyses.

Aim 1 is discussed in part one of Chapter 3. Aim 2 is demonstrated in part two of Chapter 3. Aim 3 is presented in Chapter 5.

\section{Overarching Framework and Key Definitions: Population Health and Health Disparities}

In the course of this dissertation, several concepts and terms are used frequently; definitions of them are supplied in this section. At this juncture, two global terms germane to this discussion are introduced: population health and health disparities. 
Kindig and Stoddart defined population health as a concept of health as "the health outcomes of a group of individuals, including the distribution of such outcomes within the group" (2003, p. 381). Kindig later defines population health more clearly as "health outcomes and their distribution in a population" $(2007$, p. 141). He further explains: "These outcomes are achieved by patterns of health determinants (such as medical care, public health, socioeconomic status, physical environment, individual behavior, and genetics) over the life course produced by policies and interventions at the individual and population levels" (2007, p. 141).

However, Kindig also defines population health as a conceptual framework. As such, population health is "A conceptual framework for thinking about why some populations are healthier than others, as well as the policy development, research agenda, and resource allocation that flow from it" $(2007$, p. 145). Thus, population health is the overarching analytical framework that will guide this dissertation. This analysis is concerned with elucidating how the patterns of determinants work in concert with each other to produce the observed health outcomes of geographic areas in question.

Another key concept in this dissertation is the definition of health disparity. The legal definition of a health disparity population was given in the Minority Health and Health Disparities Research and Education Act United States Public Law 106-525 (2000). It states: "A population is a health disparity population if there is a significant disparity in the overall rate of disease incidence, prevalence, morbidity, mortality or survival rates in the population as compared to the health status of the general population" (p. 2498).

While this definition is somewhat useful, the definition is circular since it uses the term to define the term. In Unequal Treatment (2003, p. 32), researchers stated: "The study committee defines disparities in healthcare as racial or ethnic differences in the quality of healthcare that are not due to access-related factors or clinical needs, preferences, and appropriateness of intervention."

This definition is limiting as well. First, given the committee's charge by the United States Congress to remove access-related factors from the committee's analysis, the IOM's definition does not consider access as a cause for disparity. The IOM study committee makes this point very clear. But from a general perspective, access is indeed a component of why disparities exist. Also, the IOM study committee argues that patient preferences do not lead to disparities when there is full and accurate understanding of treatment options among racial and ethnic minorities. However, as discussed in this work, new the theory posits that preferences can indeed contribute to disparities in health whether or not there is full and accurate understanding of treatment options among racial and ethnic minorities, because preferences are also shaped by culture and historical experiences. Because of these two issues, the IOM definition was not used in this research.

Thus, to provide clarity, this study used the following Kindig definitions: 
- Health inequality: A generic term designating differences, variations, and disparities in the health of individuals and groups

- Disparity: Inequality or difference, as in rank, amount, or quality

- Health inequity: Those inequalities in health deemed to be unfair or to stem from some form of injustice. The dimensions of being avoidable or unnecessary have often been added to this concept

In this dissertation, the term 'health disparities' is used in order to maintain conventional usage. However, when the author uses the term, the definition of health inequity is implied and thus 'health disparities' in this dissertation is defined by the author as: those inequalities in health deemed to be unfair or to stem from some form of injustice. The dimensions of being avoidable or unnecessary have often been added to this concept.

Other important terms used in this dissertation are defined next. Some terms are discussed in the literature review in Chapter 2. The remaining terms are connected to the new conceptual framework developed in Chapter 3.

\section{Key Definitions}

- Collective efficacy-Defined by Altschuler and colleagues (2004, p. 1220) as not only the social support neighbors share with each other, but also "the degree to which neighbors are willing to utilize their social cohesion to intervene on behalf of the common good."

- Collective health stewardship -Defined by the author as people in a community taking collective responsibility for the multiple behavioral determinants of health, by improving access to and quality of healthgenerating facilities (e.g. grocery stores with fresh fruits and vegetables, parks and fitness facilities, etc.) and reducing access to health-negating facilities (e.g., pollution sites, an oversupply of fast food restaurants, liquor stores, etc.).

- Cosmology —Defined by the Merriam-Webster Online Dictionary as: "1(a) a branch of metaphysics that deals with the nature of the universe and 1(b) a theory or doctrine describing the natural order of the universe."

- Cultural competence-Discussed by Nunez and Robertson (2006, p. 371) in Multicultural Medicine and Health Disparities as follows: "Cultural competence has been described, variously, as knowledge, attitude, and skills (educational perspective) about health-related beliefs and cultural values (socioeconomic perspective), disease incidence and prevalence (epidemiologic perspective), and treatment efficacy (outcomes perspective)." 
- Discrimination —Defined by Dividio et al. (2008, pp. 478-479) as: "an unfair or unjustified group-based difference in behavior that systematically disadvantages members of another group."

- Ecosocial th eory-Nancy Krieger (2001, p. 672) wrote: "Focused on the guiding question of 'who and what drives cur rent and changing patterns of social in equalities in health,' the ecosocial approach...fully embraces a social production of disease perspective while aiming to bring in a comparably rich biological and ecological analysis" (emphasis hers).

- Ethnicity -A term encapsulating culture, history, and identity among human populations. When referring to human populations as ethnic groups, I use terms such as "African Americans" or "European Americans." Using the definition provided by Dressler, Oths, and Gravelee (2005), ethnic groups within a society will incorporate any of a number of dimensions that can be placed into three broad categories - the cultural, the ancestral, and the referential - the salient features of which will vary between groups. Dressler and colleagues (2005, p. 244) define ethnicity in the following manner:

The cultural includes shared models for both the mundane (e.g., language use, diet, dress, marriage rituals) and the more abstract (e.g., concepts of self, supernatural beliefs) aspects of life. A sense of shared ancestry includes territorial homeland, common history (which may include ethnoracial discrimination), and kinship (whether construed biologically), which may or may not incorporate phenotypic or genotypic characteristics such as hair type, body build, or skin tone. With respect to the referential, as ethnic group labels fundamentally separate people into in- and outgroups (i.e., "we" versus "they"), personal (or self-defined) and social (or other-defined) identity is an integral component of ethnic definition. Folk racial categories may then be indexed here, when relevant, as an emic selfcategorization or as an eticly imposed descriptor used by others.

- Health cosmology - Defined by the author as the view of the world of health and health care which is rooted in an individual's or population's ethnicity.

- Health locus of control (LOC) —Defined by Voils and colleagues (2005, p. $158)$ as "beliefs about the controllability of health.... Individuals may believe their illness is controlled by themselves (internal LOC), powerful others (external LOC), or fate/chance (chance LOC)."

- Health stewardship - Defined by the author as views of who and/or what control health. 
- Individual health stewardship —Defined by the author as "an individual taking personal responsibility for the multiple behavioral determinants of health, whether it is improving dietary intake, increasing and maintaining a regular exercise regimen, and avoiding unhealthy habits such as smoking (all under the guidance of a trained health care provider)."

- Interpersonal trust - Defined by Vanessa Northington Gamble in Multicultural Me dicine and Health Disparities (2006, p. 437) as "trust in individuals such as physicians and other health-care professionals."

- Race-In common usage, refers to genetic differences between human populations, usually based on phenotypical differences such as skin color. In this dissertation, when referring to potential human populations in terms of ancestry and by phenotypical differences, the author uses phrases such as "people of African descent" or "people of European descent." Of race, in Unequal Treatment, authors W. Michael Byrd and Linda A. Clayton (2002, p. 491) wrote:

By the middle third of the twentieth century, traditional biology- and anthropology-based ideas of race and "races of man" that had become dominant during the nineteenth-century rise of science began breaking down. This occurred as more objective anthropologic, genetic, paleontologic, archeologic, linguistic, biogeographic, and DNA and other molecular biologic studies proved: 1) the unity of the human species, 2) the common African origins of all racial groups, and 3) the biologic insignificance of the old parameters of racial classification such as skin and eye color, hair texture, physical features, and skull size and shape....

- Racism - Defined by Van den Berghe in Unequal Treatment (2002, p. 492) as: "any set of beliefs that organic, genetically transmitted differences (whether real or imagined) between human groups are intrinsically associated with the presence or the absence of certain socially relevant abilities or characteristics, hence that such differences are a legitimate basis of invidious distinctions between groups socially defined as races"; racism is also defined by Camara Jones (2008, p. 501) as: "a system of structuring opportunity and assigning value based on the social interpretation of how one looks."

- Religiosity-Ark and colleagues (2006, p. 21) summarized religiosity in the following manner: "Levin et al. delineate three dimensions of religiosity: subjective (intrinsic) religiosity, organizational religious behavior, and nonorganizational religious behavior. Examples of organizational religious behaviors include religious attendance, membership, and affiliation, and nonorganizational behaviors include praying and reading religious materials"; religiosity is also defined by Wikipedia as: "a comprehensive sociological term used to refer to the numerous aspects of religious activity, dedication, and belief (religious doctrine)." 
- Social capita -As described by Altschuler and colleagues (2004, p. 1220), "social capital has been used to describe civic and interpersonal trust, civic participation, social cohesion, and collective efficacy."

- Social cohesion-Defined by Altschuler and colleagues (2004, p. 1220) as "the degree to which neighbors share affective and instrumental support with one another."

- Social trust - Defined by Vanessa Northington Gamble in Multicultural Medicine and Health Disparities (2006, p. 438) as "trust in collective institutions such as hospitals, health plans, or health-care professions. Social trust usually arises not only as a result of personal interactions, but also from collective relationships, media portrayals, and historical experiences."

- Temporal orientation - Burns and Dillon (2005, p. 175) wrote: "Temporal orientation is defined as the predominant cognitive, affective, and behavioral orientation to the past ("dwelling in the past"), present ("living for now"), or future ("always planning for tomorrow")."

- $\quad$ Trust -Defined by Rotter in Multicultural Medicine and Health Disparities (2006, p. 437) as "an expectancy held by a person or group that the word, promise, verbal or written statement of another person or group can be relied upon." 


\section{CHAPTER 2: LITERATURE REVIEW}

This chapter is divided into three parts. In the first section, important theories, frameworks, and concepts that contribute to the development of the new conceptual framework in this dissertation are discussed succinctly. These theories, frameworks, and concepts have deep roots in several academic disciplines. The second section of this chapter reviews the literature and discusses the four factors where key differences in the view of the world of health and health care may vary by ethnic groups. The third section is a review of the literature for the methods used in this dissertation: multilevel or hierarchical linear modeling and geographic information science (GIS).

All initial literature searches were conducted in 2008 and 2009 using PubMed (National Center for Biotechnology Information et al., 1996) and Reference Manager (ISI ResearchSoft, 2004) to identify peer-reviewed articles on the topic. In April 2010, the author utilized the Scopus database (Elselvier Science Publishers, 2004) to track any new journal article that may have reported new findings. Nearly all research articles covered in this literature review were published between 1990 and 2010.

\section{Part I: Disciplinary Explanations of Differences in Population Health}

\section{Genetics/Genetic Epidemiology}

A major contention of this dissertation is that race is valid as a social construct, but is no longer valid as a biological construct. Researchers in the field of genetics and genetic epidemiology have reported several critical findings in support of this contention. While genetics involves the study of human genes, Duncan Thomas (2004, p. 3) defined genetic epidemiology has been defined as: "...the study of the joint action of genes and environmental factors in causing disease in human populations and their patterns of inheritance in families."

Findings from these fields are important in establishing the validity of the race as a social construct argument. Research in this area is quoted at length to demonstrate the robustness of findings in this area and to clearly outline the arguments against a biological construction of race. The framework developed in this dissertation rests heavily on the findings discussed by geneticists and genetic epidemiologists. There are four major arguments that invalidate race as a biological construct. These arguments are outlined in the following four sections and then summarized in the section with the heading "Implications of Research in Genetics and Genetic Epidemiology."

\section{The Interaction of Genes with the Environment}

Genetic researchers have found that very few diseases are caused by a single gene or even a cluster of genes. Instead, what has been found is that the genes of human 
populations interact with the multiple environments in which humans live. Pearce and colleagues (2004, p. 1070) summarize this research when they stated:

The constant interaction between genes and the environment means that few diseases are purely hereditary (even if they are genetic). Purely hereditary diseases are very rare (1/2300 births for cystic fibrosis, 1/3000 for Duchenne's muscular dystrophy, and 1/10,000 for Huntington's disease) and account for a small proportion of overall disease.

Thus, without accounting for the physical or built environments and the neighborhoods in which human populations live, no comprehensive understanding of disease causation can take place. The Race, Ethnicity, and Genetics Working Group (2005, p. 525) echoed this point as well:

The difficulty that has been encountered in finding contributory alleles for complex diseases and in replicating positive associations suggests that many complex diseases involve numerous variants rather than a moderate number of alleles, and the influence of any given variant may depend in critical ways on the genetic and environmental background.

\section{The Role of Lifestyle/Behaviors in Disease Causation}

Diseases are not only influenced by the environments of human populations, but the lifestyle or behaviors of human populations. Especially with chronic diseases such as hypertension, diabetes, and heart disease, lifestyle factors such as physical exercise, smoking, alcohol consumption, and dietary patterns play a key role in the generation of disease patterns. Kaprio (2000, p. 1257) stated:

While the role of genetic factors in diseases such as hypertension, asthma, and depression is being intensively studied, family studies and the large geographical and temporal variation in the occurrence of many diseases indicate a major role of the environment. It is often assumed that diseases are genetic because they run in families, but this often reflects a common environment and lifestyle rather than a genetic influence.

\section{The Lack of Alleles or Polymorphisms Concentration within "Races"}

In addition to the contribution of environmental and lifestyle factors, the lack of allele and polymorphism concentration within commonly described human races-Black, White, Asian, Native American, etc.- - is also a damaging argument against the biological construction of race. Rosenburg and colleagues (2002, p. 2381) reported:

Of 4199 alleles present more than once in the sample, $46.7 \%$ appeared in all major regions represented: Africa, Europe, the Middle East, Central/South Asia, East Asia, Oceania, and America. Only 7.4\% of these 4199 alleles were exclusive to one region; region-specific alleles were usually rare, with a median relative frequency of $1.0 \%$ in their region of occurrence. 
Researchers of polymorphisms among human populations also report similar findings. Jorde (2000, p. 979) wrote: "Recently, surveys of mitochondrial, Y-chromosome, and various types of autosomal polymorphisms have all shown that most human genetic diversity is found within, rather than between, populations."

In their article, Barbujani and colleagues (1997, p. 4516) also argued:

It is often taken for granted that the human species is divided in rather homogeneous groups or races, among which biological differences are large. Studies of allele frequencies do not support this view, but they have not been sufficient to rule it out either. We analyzed human molecular diversity at 109 DNA markers, namely 30 microsatellite loci and 79 polymorphic restriction sites (restriction fragment length polymorphism loci) in 16 populations of the world. By partitioning genetic variances at three hierarchical levels of population subdivision, we found that differences between members of the same population account for $84.4 \%$ of the total, which is in excellent agreement with estimates based on allele frequencies of classic, protein polymorphisms. Genetic variation remains high even within small population groups. On the average, microsatellite and restriction fragment length polymorphism loci yield identical estimates. Differences among continents represent roughly $1 / 10$ of human molecular diversity, which does not suggest that the racial subdivision of our species reflects any major discontinuity in our genome.

Thus, the distribution of alleles and polymorphisms among human populations do not correspond to the commonly described classification of human races. As stated, there is more genetic variation within the commonly described human races than there is between them. This means that the concentration of diseases within the commonly described races of human populations is not likely to occur due to a genetic influence.

\section{Genetic Variation in Human Populations Explained by Geographic Distance, Not Race}

Researchers have also argued that the genetic variation among human populations is not explained by a biological construction of race, but rather geographic distance. Geographic distance would explain the genetic variation of human populations due to dispersion of human populations around the world and their subsequent genetic adaptations to their diverse environments. In summarizing the literature pointing to this conclusion, Goodman (2000, p. 1700) wrote:

Starting with Lewontin, 13 studies have statistically apportioned variation in different genetic systems to different levels among "races" and within "races" and smaller populations such as the Hopi, the Ainu, and the Irish. Lewontin collected data on blood group polymorphisms in different groups and races. He found that blood group variation among races statistically explained about $6 \%$ of the total variation. The implication of Lewontin's results is that if one is to adopt a racial paradigm, one must acknowledge that race will statistically explain only a small proportion of variations. These variations are better explained by geographic distance. 
In extending this argument, Bamshad and colleagues argue that genetic variation among human populations has little to do with skin color. Bamshad and colleagues (2003, p. 578) wrote:

Group membership has commonly been assigned by place of birth (e.g., Africa, Japan), religious belief (e.g., Amish, Jewish, Hindu), language (e.g., Amerind, Khoisan), or physical traits (e.g., skin color). These proxies vary in the extent to which they reflect demographic trends or evolutionary forces that affect the distribution of neutral genetic variation. As a result, the concordance of each of these proxies to population structure inferred from neutral genetic data also varies. For example, an ethnic label such as "Mbuti" is an accurate guide to population structure, because it delimits a group that has differentiated from others as a result of reproductive isolation and genetic drift. In contrast, a proxy such as skin color is inaccurate, because it delimits a group (e.g., sub-Saharan Africans, New Guinea highlanders, and Australian aborigines) whose members are similar, vis-a-vis this trait, as a result of convergent natural selection.

Bamshad and colleagues (2003) make another important point here. The ways by which people are assigned to certain groups, especially when physical traits are used in racial assignment, poorly correlate with genetic variations. Skin color, which is the foundation for racial assignments, is an inaccurate proxy of genetic variation. Again, genetic variation in human populations is caused by geographic variation and a result of reproductive isolation and genetic drift.

\section{Implications of Research in Genetics and Genetic Epidemiology}

Given the nature of the findings in genetics and genetic epidemiology, there is no firm foundation upon which to rest the argument of a biological construction of race. Not only are there confounding factors that must be considered in disease causation (i.e. environment and lifestyle), but also the distribution of alleles and polymorphisms do not support a biological rationale for disease causation within the commonly described racial groups. In addition, skin color is a poor proxy for the genetic variation among human population that is due to geographic dispersion. Thus, there is little basis upon which to base arguments for certain genes in commonly described racial groups that would cause them to bear a disproportionate burden of morbidity and mortality in societies around the world.

It is important to highlight the implications of practicing medicine based on skin color, which is the basis for assigning people to the commonly described racial groups. Bamshad and colleagues (2003, p. 578) wrote:

A major goal of biomedical research is to develop the capability to provide highly personalized health care. To do so, it is necessary to understand the distribution of inter-individual genetic variation at loci underlying physical characteristics, disease susceptibility, and response to treatment. Variation at these loci commonly exhibits geographic structuring and may contribute to phenotypic differences between groups. Thus, in some situations, it may be important to 
consider these groups separately. Membership in these groups is commonly inferred by use of a proxy such as place-of-origin or ethnic affiliation. These inferences are frequently weakened, however, by use of surrogates, such as skin color, for these proxies, the distribution of which bears little resemblance to the distribution of neutral genetic variation.

Yet, with a plethora of research detailing the existence of health disparities between commonly described racial groups, it is clear that race matters in some sense. But the key to unlocking the puzzle of health disparities is precisely in understanding how race matters. If arguments for a biological construction of race cannot or do not explain variations in health outcomes among human populations, then understanding an alternative construction of race is a necessity. The social construction of race-how race (based on skin color or some physical feature) is viewed and interpreted within in any given society - may provide the key to explaining why certain human populations bear a disproportionate burden of disease and others do not. To understand the social construction of race and how social determinants play a role in producing health outcomes, we now turn our attention to the social sciences.

\section{Sociology/Medical Sociology}

Several important concepts have emerged from the field of sociology as it relates to the social determinants of the health of human populations. Sociologists have been at the forefront of the movement to understanding the role of social determinants in producing or predicting health outcomes, especially by highlighting the roles of social bonds, culture, and history. Additionally, sociologists were among the first to discuss how the variable of race is confounded with other social phenomena, as discussed in Chapter 1. Collectively, the contributions of sociologists strongly inform the construction of the new conceptual framework.

One of the most venerable sociologists - and perhaps the greatest social scientistwas William Edward Burghardt Du Bois. In his groundbreaking work The Souls of Black Folk, Du Bois not only articulates the proposition of the dual identity of persons of African descent in America (using the term "double consciousness"), but also anticipates measures that would explain how the social construction of race would be needed to explain important social outcomes. Du Bois (1903, p. 119) wrote: "We feel and know that there are many delicate differences in race psychology, numberless changes that our crude social measurements are not able to follow minutely, which explain much of history and social development." By discussing "differences in race psychology," Du Bois foresaw a time when social scientists will understand how people who are assigned to different racial groups in a given society view the world, and are viewed by the world, in different ways that impact their behaviors and their outcomes.

More recently, sociologists have also discussed ways in which ancient medicine resembles and precedes modern interactions of providers and patients. Sociologist Doris Y. Wilkinson highlighted the significance of faith and trust in the health provider when comparing contemporary medicine to ancient medicine. Wilkinson wrote: 
Universally, the healing arena incorporates the relational imperatives of trust and faith in those on whom one depends for care.... The traditional healer-patient status-role set, resembling the modern healer-patient dyad was founded on these and other cultural norms and values, complementary psychology responses, and demographic factors as well (Watson 1998, p. vii).

Thus, Wilkinson characterized traditional medical practices by: “...successful past experience, even if it is infrequent; the in-group affiliation and identity of the healer; acceptance of the wisdom of the practitioner; medical folklore; and shared beliefs about the etiology of illness..." (Watson 1998, p. vii).

In these statements, Wilkinson posited that the practice of medicine is situated in a cultural and psychological context. Another sociologist-Wilbur Watson-echoed Wilkinson when comparing the traditional or folk medical practitioner and the modern medical doctor (Watson 1998, p. 15).

The chief task of the folk medicine practitioner is to aid the sufferer in mobilizing his psychological, spiritual, and bodily resources to return to a state of well-being. In this task, the folk medicine practitioner and the modern medical doctor have a common goal. They differ mainly in their assumptions about the nature of health and illness, theories of disease, techniques of intervention for purposes of treatment, knowledge of and value assigned to modern medical technology, and approaches to preventing illness.

Watson (1998, p. 94) goes on to describe the problems that the differences in assumptions pose for the modern health provider: "...medical students and physicians do not, as a rule, study the backgrounds and cultures of their patients, nor the bearing of world views on health care and the hypothetical significance of congruence on health care delivery and patient compliance."

As mentioned previously, sociologists have also posited that factors such as social cohesion, collective efficacy, and social capital affect health outcomes. Researchers have explained that these factors represent the strength of the social bonds in a given community and are a resource for the health of persons in a given neighborhood. Researchers of social cohesion, collective efficacy, and social capital have also tested these factors empirically in the literature with mixed findings.

\section{Health Economics}

Important concepts or frameworks developed in the field of health economics help elucidate our understanding of health and the ways in which we should think about the allocation of resources to the health care system. Folland and colleagues $(2007$, p. 8) define health economics as: "the study of the allocation of resources to and within the health economy." One of the primary insights of health economics is to elucidate how the provision of health care is different from most other goods and services in a market. 
Health care in the United States differs from the provision of most other goods and services in the following ways, according to Folland et al. (2007):

- The presence and extent of uncertainty (surrounding the timing \& intensity of illness)

- The prominence of insurance

- Problems of information or information asymmetry

- The large role of nonprofit firms

- Restrictions of competition

- The role of equity and need

- Government subsidies and provision

Since health care is different in these important ways, strategies in dealing with issues of the cost, access, and quality of health care must take these differences into account.

Health economists have also contributed various concepts, such as the production function of health, an equation that specifies the following:

$$
\text { Health Status }=f(\text { Health Care, Lifestyle, Environment, Human Biology })
$$

In other words, health status is a function of four distinct inputs: health care, lifestyle, environment, and human biology. This equation forms the basis for the model depicted in Figure 1.

Health economists Darrell Gaskin, Alvin Headen, and Shelley White-Means also introduced the idea that the legacy effects of slavery, racism, and discrimination have negative inter-generational effects on the health of African Americans-specifically, in the differential capacity of racial/ethnic groups to invest wealth in health and health care.

Finally, health economists have conducted research dealing with preferences for hypothetical disease states. Research in this area, involving concepts such as risk attitudes (as they relate to health) and the Von Neumann-Morgenstern Expected Utility Model vs. the Kahneman-Tversky Prospect Theory, are discussed in more detail in the second part of this chapter.

\section{Social Epidemiology}

Like sociologists, social epidemiologists have played a major role in helping researchers understand the full scope of the manner in which social determinants play a major role in determining health outcomes. According to Nancy Krieger (2001), social 
epidemiologists have developed three important frameworks to explain how social factors impact the health of populations. These frameworks are:

1) Psychosocial theory

2) Social production of disease and/or political economy of health

3) Ecosocial theory and related multi-level dynamic perspectives

Psychosocial theory, according to Krieger (2001, p. 674), “...directs attention to endogenous biological responses to human interactions. Its focus is on responses to "stress" and on stressed people in need of psychosocial resources." Alternatively, the social production of disease and/or political economy of health framework is described in the following manner by Krieger (2001, p. 670): "The underlying hypothesis is that economic and political institutions and decisions that create, enforce, and perpetuate economic and social privilege are root-or 'fundamental'-causes of social inequalities in health."

Ecosocial theory, as opposed to the first two frameworks, considers the effect of determinants from multiple levels. Where psychosocial theory focuses more on the individual and the social production of disease and/or political economy framework focuses on social determinants, ecosocial theory focuses on the ways in which the individual is embedded within social structures and processes. Ecosocial theory is, therefore, multilevel in scope and "focused on the question of "who a nd what drives current and changing patterns of social inequalities in health'...” (p. 672).

Krieger also writes that: “...no aspect of our biology can be understood absent knowledge of history and individual and societal ways of living" (p. 672). Ecosocial theory also pays attention to the pathways of embodiment, or put another way, the ways in which our biology is affected by our social and physical environments - both in the present and due to the cumulative impact of past history. The new conceptual framework proposed in this dissertation can be characterized as an ecosocial conceptual framework.

\section{Psychology/Cultural Psychology}

Several important concepts in the field of psychology have been utilized in health services research as a possible factor in determining health outcomes. Some of the concepts are: health locus of control, temporal orientation, and body image. These concepts, and their contribution to the new conceptual framework, will be discussed in more detail in the second part of this chapter.

However, it is in the sub-field of cultural psychology that critical race theories have been proposed. Cultural psychology is defined as: "symbolic representations that condition and follow from behavior, giving rise to characteristics ways of perceiving, understanding, anticipating, valuing and behaving for members of a socially defined group” (Jones 2002, p. 1). Cultural psychologists such as James M. Jones, Ivory Lee 
Toldson, and Ivory Achebe Toldson have advanced arguments that essentially state that history and culture shape the psychology of African Americans in ways that are different from European Americans.

In advancing this argument, Toldson and Toldson (2001, p. 403) stated: "Two forces come together to make up the fabric of African-centered psychology: (a) African consciousness - the primary force - and (b) racism and oppression - the second force." Jones (2002, p. 11) followed: "The historical evolution of African American culture has resulted in large part from the coping-adaptation sequences that derive both from African origins (evolutionary) and challenges to racism in the American context (reactionary). As a result, the psychology of African Americans is heavily context dependent." In other words, history has helped shaped African American psychology (via racism, slavery, discrimination, and segregation) along with an alternative cultural orientation (African origins).

\section{Religion or Psychology of Religion}

Three concepts in the field of religion or the psychology of religion have been proposed in previous research as possible explanatory factors in determining health outcomes: religiosity, religious coping, and spiritual health locus of control. Religiosity literature examines the impact of religion, religious beliefs, and religious practices on health behaviors, medical utilization, and health outcomes. This body of literature, and its contribution to the new conceptual framework, is discussed further in the second part of this chapter.

\section{Health Geography/Spatial Epidemiology}

The field of health geography/spatial epidemiology, or the study of spatial factors that impact health, has greatly enriched the study of factors that contribute to health outcomes. Spatial epidemiology is defined as: "the description and analysis of geographic variations in disease with respect to demographic, environmental, behavioral, socioeconomic, genetic, and infectious risk factors" (Elliot \& Wartenberg 2004, p. 998).

In the field of health geography, several factors have been proposed to help explain variation in health outcomes among people in different geographical areas. In terms of access, Guagliardo (2004) has highlighted concepts such as provider supply, distance to provider, and spatial accessibility as ways to measure the impact of geography on health. Other studies examine the impact of distance to pollution sites (Williams et al. 2007) or the differential access to services and amenities (i.e. grocery stores, food desert classifications, parks and exercise facilities). Another area of research involves studies that examine contextual effects of neighborhoods where usually a summary measure is used to characterize an entire area (i.e. neighborhood socioeconomic status). These studies often use small area analysis or area-to-area comparative analysis. Neighborhoods' contextual effect has also been examined using multilevel or hierarchical 
linear modeling to assess individual level and neighborhood contextual factors simultaneously.

\section{Health Services Research or Health Disparities Research}

In health services research or research investigating health disparities, two dominant models have been proposed to discuss variation in health outcomes among various populations. Health services researchers have extensively used the Anderson and Aday model, while health disparities researchers have used Institute of Medicine model (both were discussed and depicted in Chapter 1). Additionally, health disparities researchers have examined trust, perceptions of quality of provider care, and discrimination. These health disparities factors are all discussed in the second part of this chapter and form a major part of the new conceptual framework.

\section{Population Health}

In the field of population health, several factors have been identified as potential causes for health outcomes among populations. The population health approach was developed in Canada and the United Kingdom, especially the Canadian Institute for Advanced Research. The population health approach is characterized by examining the following list of determinants or reasons for health heterogeneities according to Evans (1995):

- Reverse causality

- Differential susceptibility

- Individual lifestyle

- Physical environment

- Social environment (and psychological response)

- Differential access to/response to health care services

As discussed in Chapter 1, the field of population health is the guiding and over-arching framework for this dissertation.

\section{Medical Anthropology}

Ann McElroy (1996, p. 1) defined medical anthropology as "the study of human health and disease, health care systems, and biocultural adaptation." In the field of medical anthropology, three different theoretical orientations have been developed to help discuss health and health outcomes among populations. Medical ecology views 
populations as biological as well as cultural units and studies interactions among ecological systems, health, and human evolution. Ethnomedical analysis focuses on cultural systems of healing and the cognitive parameters of illness. Finally, applied medical anthropology deals with intervention, prevention, and policy issues and analyses the socioeconomic forces and power differentials that influence access to care.

Among the key insights of medical anthropology is the understanding of folk medicine and the ways by which folk medicine differs from Western medicine. With this, according to McElroy (1996, pp. 4-5), it is understood that:

Pluralistic societies often encompass several ethnomedical systems. Among these are cosmopolitan medicine, a dominant system in North America and in urban centers elsewhere, which emphasizes empirical research, naturalistic explanations, technology and surgery, use of extraordinary intervention to preserve life, and hierarchical roles. Humoral medicine, derived from ancient Greek medicine, emphasizes that health reflects balance among bodily humors and their intrinsic qualities. Disequilibrium derives from ingestion of inappropriate food and other substances, from change of climate, and from exposure to natural elements like air and water. Therapy involves restoring equilibrium through applying or ingesting remedies opposite to the state of the body. Humoral medicine coexists with other systems in Latin America, the Middle East, Malaysia, Indonesia, and the

Philippines. Ayurvedic medicine in India and Chinese traditional medicine meld humoral elements with elements of other systems.

Clearly then, different ethnic groups around the world have different ways of conceptualizing medicine. This proposition is a central feature of the new conceptual framework.

\section{Cultural Anthropology}

Cultural anthropology contributes the definition for ethnicity that is used in this dissertation Dressler and colleagues (2005). The new conceptual framework is also informed by cultural anthropologist Melville Herkovits' work (1941) that demonstrated the influence of African culture on persons of African descent in America. Additionally, the socio-cultural school also discussed the ways by which past historical experiences plays a role in determining health outcomes. Outram and Ellison (2006, p. 84) wrote:

...[A]nthropological critiques from the socio-cultural school focus on the social construction of race/ethnicity and place greater emphasis upon how race/ethnicity emerged historically and has been associated with inequalities in power due to colonialism, slavery, and discrimination. As such, the view of race/ethnicity sees disparities in health and related biological characteristics as the consequences of hierarchical socio-cultural and political practices rather than the result of innate genetic differences. 
The implications of which view dominates scientific research and the practice of medicine cannot be overstated. Outram and Ellison (2006, p. 98) continued:

In the meantime, what are the likely consequences for public health of this predilection for genetic explanations of racial/ethnic inequalities in disease and disease risk? First, it can lead public health to view racial/ethnic health inequalities as the products of intractable genetic differences that are not susceptible to public health interventions. Second, it may encourage the development of different public health services for different racial/ethnic groups-further stigmatizing some groups and re-enforcing notions of innate differences. And, third, it may distract attention away from analyses that explore the political economy of race/ethnicity and its role in structural violence through historical and contemporary discrimination.

Thus, there are real consequences to health professionals engaging in the practice of public health, medicine, and health research while embracing the biological construction of race. When health professionals operate under such a paradigm, proposed programs, solutions, and medical interventions may actually exacerbate the problem of health disparities by ignoring the critical evidence found across a wide range of the scientific literature.

Yet, it is not enough to simply state that the social construction of race provides an alternative explanation without detailing how the social construction of race, along with cultural and historical factors, help produce variations in health outcomes. The following discussion of health cosmology factors - in concert with the first part of Chapter 3provides insight into measures that capture the ways by which people view the world of health and health care, which influences health behaviors, medical utilization, and ultimately, health outcomes.

\section{Part II: Review of Health Cosmology Literature}

In second half of this chapter, the range of the literature review will be narrowed to and focused primarily on previous empirical research which points to the existence of critical differences in the way in which diverse racial and ethnic groups view the world of health and health care-highlighting the roles of culture, historical experiences, and social race assignment (via discrimination). Evidence in psychology, religion, economics, health services, and health disparities peer-review literature demonstrates that people of African descent view or perceive the world of health and health care in the United States in a fundamentally different way from people of European descent. There are four factors that point to the existence of a health cosmology that may vary by ethnicity:

1) Health stewardship (views of 'who' and/or 'what' controls health)

2) Views of diseases and/or the body 
3) Medical trust (future looking based on past)

4) Perceptions of provider or system quality (backward looking to personal experience)

The "health stewardship" factor includes measures of religiosity and health locus of control. The "views of diseases and/or the body" factor includes measures found in health economics, psychology, and medical anthropology. This dissertation focuses on the preference-based scores for health states found in health economics' cost-utility literature. The "medical trust" factor includes measures of interpersonal trust in health care providers and social trust in the health care system. Finally, the "perceptions of provider or system quality" factor includes the following measures:

- Satisfaction with the medical encounter

- Time spent with physician

- Wait time to see physician

- Communication, comprehension, and linguistic barriers

- Social distance or race concordance

- Cultural competence

- Perception of bias or discrimination

The literature involving each of these factors is discussed at length in the following section. Researchers in these areas report statistically significant differences in the way that people of African descent and people of European descent in the United States view the world of health and health care. When viewed collectively, the following factors have a large impact on how we structure and deliver the service of health care in a large variety of health care systems around the world.

\section{Racial/Ethnic Differences in Health Stewardship}

\section{Health Locus of Control Literature}

Several articles find that there are ethnic differences in health locus of control measures. In a study of 167 children, Malcarne et al. (2005) used the Multidimensional Health Locus of Control (MHLC) and found that Latino and African American youth possessed stronger beliefs in chance than European American youth. They also found that European American youth were less likely than African American youth to have a belief in powerful others. Even among children from wealthy families, African American youth endorsed stronger beliefs in powerful others and chance when compared to Latino and European American youth. In a sample of 214 children, Wilson et al. (1994) found 
that African American boys endorsed significantly higher external health locus of control beliefs (in powerful others) than European American boys or girls.

In another study that used convenience sampling, Ayalon and Young (2005) compared 70 Blacks and 60 Whites aged 18 years or older at a community college in large Midwestern city. Using regression analysis, they reported: "The results indicated significant racial group differences in external locus-of-control beliefs (in God, Powerful Others, and Chance)... with Blacks reporting beliefs in external control significantly more than Whites..." (p. 398).

In a comparison of 152 African American women and 197 European American women in South Florida ranging from ages 19 to 93, Barroso et al. (2000, p. 268) found that "African-American women were significantly more likely to believe in chance, or to depend on powerful others for their health." In a study of African American and European American women with breast cancer, Bourjolly (1999) found that both groups of women reported similar external beliefs in health locus of control. Using the MHLC scale, Bekhuis et al. (1995) found ethnic differences between 197 African Americans and European Americans in the rural South in terms of belief in God control after controlling for the effect of education.

In a comparison of 690 with chronic fatigue syndrome, Buchwald (1996) found no significant racial/ethnic differences in health locus of control measures. In another study of 587 participants in Harris County, Texas community health centers, Arrufo et al. (1993) found Latino Americans and African Americans had a higher external health locus of control when compared to European Americans. Arrufo and colleagues used the MHLC.

In a study of 342 community dwelling elderly individuals using the MHLC Subscale, Galanos et al. (1994) found that being European American predicted a lower score on the Chance Subscale when compared to African Americans and controlling for age, education, and gender.

\section{Religiosity and Religious Coping Literature}

Several articles find racial/ethnic differences in religiosity or religious coping. For instance, in a study of 6,082 persons aged 18 and over (in a sample of 3,570 African Americans, 891 non-Hispanic whites, and 1,621 Americans of Caribbean descent), Taylor, Chatters, and Jackson (2009, pp. 334-335) found the following using regression analysis:

Overall, these analyses indicated that African Americans and Black Caribbeans had significantly higher levels of spirituality than non-Hispanic Whites for both importance and self-assessed spirituality. These differences persisted even after the application of controls for demographic and denominational factors that are differentially distributed within Black and White populations are known to be of consequence for religious involvement. 
They also found no significant differences in importance of spirituality and self-assessed spirituality between African Americans and Black Caribbeans.

Halbert and colleagues (2007) examined differences in religiosity among 50 African American men and 69 European American men with newly diagnosed prostate cancer in Philadelphia, Pennsylvania. The mean age of the sample was 63.7 years. They found that, "African American men reported significantly greater levels of religiosity compared with European American men" (p. 281). Halbert et al. used multivariate regression analysis and controlled for sociodemographic factors, clinical factors, and cultural values. African American men also were more likely to report a present temporal orientation compared to European American men. A present temporal orientation means that a person places more weight on the present than the future, while a future temporal orientation means that a person places more weight on the future than the present.

In a study conducted by Taylor, Chatters, and Jackson (2007), researchers examined a subsample of persons over the age of 55 from the National Survey of American Life using a national multistage probability design. Out of a sample of 837 African Americans born in the US, 302 African Americans born in or who migrated from the Caribbean, and 292 European Americans, the researchers used 16 different measures of religious and spiritual involvement. They grouped the 16 measures into five main categories: organizational religious participation, non-organizational religious participation, subjective religiosity, spirituality and religious coping. Using 16 different regression equations, the researchers found that African Americans born in the US reported significantly higher religious and spiritual involvement in 15 of the 16 categories while African Americans born in or who migrated from the Caribbean reported significantly higher religious or spiritual involvement in 13 of the 16 categories.

In a sample of 31 African American and 29 European American women from a metropolitan area in Minnesota on hemodialysis for end-stage renal disease, ranging from ages 24 to 79, Tanyi and Warner (2007) reported that African American women scored higher on religious well-being using the spiritual well-being scale when compared to European American women (using Student's $t$ tests). In another study, Ark and colleagues (2006) examined the religiosity, religious coping styles, and health service use of women living in Nashville, Tennessee ranging from ages of 55 to 95 . Out of a sample of 159 European American and 115 African American women who lived in publicly subsidized high-rise community apartments, Ark and colleagues found that African American women reported significantly higher scores on measures of religiosity and religious coping styles.

African American women were more likely to report self-directed and deferring religious coping styles while European American women were more likely to report selfdirected and collaborative religious coping styles. Using multivariate Poisson regression, Ark and colleagues estimated the effects of religiosity and religious coping styles on the health service use of European American and African American women. While subjective religiosity led to significantly lower physician visits among European American and non-organizational religious behavior led to lower physician visits among 
African American women, European American women's self-directed coping style led to significantly greater utilization of physician visits, emergency department visits, and hospital inpatient days while for African American women, self-directed coping style led to significantly lower utilization of physicians visits and emergency department visits. According to Ark and fellow researchers (2006, p. 27), these findings may indicate that: "The distinct patterns in the effects of religious coping styles on health service use outcomes between White and African American women suggest the same coping styles may function differently in each group."

In the study covered in the previous section, using a sample of Midwestern community college students, Ayalon and Young (2005, p. 399) also found:

...Blacks used psychological or social services less frequently and religious services more frequently than did Whites. The present study suggests that even after controlling for the level of education and the level of distress, Blacks are less likely to use psychological or social services than Whites. Furthermore, the entire sample had relatively easy access to service through student health facilities, and therefore, access barriers cannot explain the difference.

Therefore, they highlight in their sample that the mode of coping for African Americans is more likely to involve the use of religious services as opposed to outpatient mental health services.

Mansfield, Mitchell, and King (2002) conducted a random-digit-dial telephone survey of adults (696 European Americans and 281 African Americans) over age 18 in eastern North Carolina. They found that African Americans were significantly more likely to "pray for guidance, help, or healing self or others" and "pray for healing own medical problems" when compared to European Americans. African Americans were reported higher percentages of people who possessed beliefs in religious miracles, God's use of religious healers to cure illness, God's ability to act through doctors to cure illness, and God's will as the most important factor in recovery when compared to European Americans. Of particular interest is the following statement by Manfield and colleagues (2002, p. 406):

Our findings are consistent with Abrum's account of beliefs and meanings of health among African-American women. She concludes that the centrality of belief and meaning of health is that the body is a gift from God, that the power to heal remains firmly in the hands of God, and that the doctor is merely the instrument of God's power.

In a telephone survey study of 1,687 pairs twins (220 minority pairs) examining alcohol use and smoking rates, Heath et al. (1999, p. 145) found-using proportional hazards regression models and genetic variance component models-that African Americans adolescents “...showed greater religious involvement (frequency of attendance at religious services) and stronger religious values (e.g. belief in relying upon their religious beliefs to guide day-to-day living)." They also concluded that religiosity 
and religious involvement displayed high heritability among African American youth but only moderate heritability among European American youth and others.

\section{Discussion}

While the health locus of control literature is not unanimous in its conclusions, there is a clear pattern that researchers that use the MHLC consistently find ethnic differences in the health locus of control measures between African Americans and European Americans. These articles find these differences at three different phases of the life span - in childhood, in adulthood, and in the elderly. The two articles that do not find a difference are articles that discuss subjects who are suffering from a disease or condition (chronic fatigue syndrome or breast cancer). This perhaps indicates that a different mechanism may be at work once people have a disease or condition as opposed to when they do not.

The religiosity and religious coping literature speaks fairly unanimously concerning key differences between African Americans and European Americans. These differences are noted in adolescent, adult, and senior populations indicating consistency across the lifespan. When combined with the health locus of control literature, we find that are found in various geographic areas across the United States from Nashville, Tennessee to South Florida to Harris County, Texas to eastern North Carolina to Philadelphia, Pennsylvania to the rural South. Taylor and colleagues also found these differences in two national surveys. Differences are found in studies even after controlling for income and education in regression analyses.

As a counterpoint, however, Fiori and colleagues (2004) point out that, for some, there is "God-mediated control" instead of a more passive "God-is-in-control" view. Fiori and colleagues (2004, pp. 393-395) define "God-mediated control" as: "equivalent to a collaborative coping style until a stressor reaches a certain threshold, at which point an individual utilizing God-mediated control must surrender to God to (paradoxically) maintain a healthy sense of control." Based on this definition, the author would hypothesize that a person with "God-mediated control" would be classified as having a more active coping stance than someone with a "deferring to God" coping mechanism, but less active than a person with a "self-directed" coping stance. Therefore, a "Godmediated control" coping stance would place an individual on a control axis between a person with a "God-is-in-control" view and an "I-am-in-control" view.

\section{Racial/Ethnic Differences in Preference-Based Scores for Health States}

\section{Cost Effectiveness and Cost Utility Analysis}

Cost effectiveness and cost utility analysis are used to help decision-makers allocate resources efficiently. Cost effectiveness analysis gives a result based on outcomes (lives saved) while cost utility analysis yields both preference-oriented results (quality of lives saved). The notion of quality denotes or suggests value or preference-not just how much longer a person lives or how much their blood pressure reduced (outcome), but also 
what is the quality of added life and how reduced blood pressure improves mobility and everyday activities (preference). Living an additional six years with a full range of mobility and sensation filled with vigor and health may be preferred to living an additional ten years with no mobility, unconscious and connected to life-support devices.

\section{Measuring Preferences}

Therefore, cost utility can be seen as going one step farther than cost effectiveness analysis because it attempts to quantify quality in some way. Cost utility analysis provides us with a mechanism to account for preferences (when decisions are made under uncertainty). Using such measures such as QALY (quality adjusted life years) or DALY (disability adjusted life years) as outcomes, cost utility analysis captures and takes into account individuals' preferences or utilities. In health care, multi-attribute preferencebased measurement tools are used to assess individual's preferences for health conditions.

According to the von Neumann-Morgenstern Expected Utility Model people choose alternatives that have the highest expected utility, not the highest expected value. A person's utility or preference is based on their view of risk aversion. Risk adverse decision-makers seek to avoid gambles involving negative outcomes. In other words, the odds must distinctly be in their favor in order to take a gamble. Risk neutral individuals neither favors nor avoids a fair gamble. Risk seeking decision-makers, on the other hand, favor gambles with negative expected values. The key insight here is that for different levels of risk aversion, expected utility differs (Frank 2003).

Risk attitudes form the foundation methods of assessing preferences for health states (Drummond et al., 2005). Using tools such as scaling response methods (i.e. rating scales), choice response methods (i.e. time trade off), and standard gamble methods, researchers are able to elicit the values or preferences of individuals. Since standard gamble is the only tool to test for decision making under uncertainty, it is the only current tool that technically captures risk attitudes being directly derived from the assumptions detailed in the von Neumann-Morgenstern Expected Utility Model (Drummond et al. 2005).

Because testing each individual using tools such as rating scales, time trade off, and standard gamble are time consuming and highly complicated, pre-scored multi-attribute preference-based systems are commonly used. The EQ-5D, Short Form 6D, and the HUI systems were each developed using choice response methods (therefore their scores are preference-based). The EQ-5D's preference scores are based on the time trade off method, while the Short Form 6D and HUI systems were scored using the standard gamble utilities.

\section{Alternative Theory}

Economists have long held that individuals make rational decisions based on which choice or gamble will derive the greatest utility for them. However, Kahneman-Tversky Prospect Theory argued that humans do not always act rationally. According to Drummond and colleagues (2005, p. 146): "[Humans] may focus too much on the near 
term; or they may be overconfident, thinking they know what they don't know, or putting too much stock in their own beliefs." Drummond and fellow researchers also (2005, p. 143) note: "It is important to appreciate that the von Neumann-Morgenstern axioms and utility theory are not intended as descriptions of how individuals actually make decisions in the face of uncertainty, but as a prescriptive or normative model of how they 'ought' to make such decisions if they wish to act rationally as defined by basic axioms." What is highlighted here is the notion that rationality does not often explain health behaviors. Instead, factors such as an individual's culture and history-"their own beliefs"-are important determinants of health behaviors, medical utilizations patterns, and ultimately, health outcomes.

\section{Structure of Review of Literature in This Section}

This literature review in this section only discusses the articles that meet two stringent criteria: 1) empirical literature in which researchers' methods were based on time trade off or standard gamble tools of assessing preferences; and 2) researchers discuss findings of similarities or dissimilarities in racial and ethnic preferences for health states. Although the time trade off method does not technically elicit utilities, it is used fairly commonly along with standard gamble tools and therefore, the articles will be included in the review.

\section{Researchers' Findings from Multi-Attribute Preference-Based Measures}

Shaw and colleagues (2007) sought to examine the determinants of preference scores for the EQ-5D with a sample size of 4,048 using a multi-stage probability design in order to sample participants for their study. The study consisted of an oversampling of "Hispanics and nonHispanic blacks," although the authors do not give precise figures for how many persons from each ethnic group participated in the study. Using multinomial logistic regression, Shaw and fellow researchers (2007, pp. 485-486) found:

The predicted values for all three racial/ethnic groups tend to be fairly similar for very mild states of health. For moderate health states, the values for blacks tend to be higher than those for the other racial/ethnic groups. For severe states of health, the values for blacks tend to be somewhat higher than those for Hispanics, while the latter tend to be higher than those for nonHispanic nonblacks.

Using a 2003 MEPS survey sample of 20,248 people who completed the EQ-5D, Fu and Kattan (2006) sought to improve upon the Lutbetkin et al. study (2005). Using more elaborate methodologies, a stronger measure for disease condition, and focusing more on race/ethnicity, Fu and Kattan (2006, p. 2446) found that significant “...differences between Blacks, Hispanics, and Whites disappear with a comprehensive control of disease conditions (by CS) and functional and activity limitations." However, they do note that: 1) the EQ-5D was scored using the time-trade off method and therefore the score is not technically a utility measure; and 2) "the construct validity of the preferencebased EQ-5D measure varies by race/ethnicity and the use of population-average preference-based scores may produce cost/QALY estimates that are not quite accurate for different racial/ethnic groups" (2006, p. 2445). 
Using a 2000 MEPS sample of 13,646 adults who completed the EQ-5D, researchers found lower scores for African Americans when compared to European Americans (Lutbetkin et al. 2005). When controlling for age, gender, race/ethnicity, income, education, and condition/comorbidity, significant differences in EQ-5D index scores were found between blacks and whites.

There were significant differences found in the EQ-5D index scores for whites when compared to Hispanics $(p=0.0015)$ and Asians/Pacific Islanders $(p=0.0015)$. No significant differences were found between whites, Hispanics and Asian/Pacific Islanders in EQ VAS scores.

\section{Researchers' Findings from Standard Gamble and Time Trade off Tools}

Wittenberg et al. (2006) examined the relationship between race/ethnicity and preference scores for a variety of hypothetical diseases. The researchers used four dataset and pooled the data according to criteria similar to the ones used to select articles for literature in this section. There were 390 total participants with ages ranging from 19 to 89 who contributed a total of 956 preference scores $-90 \%$ of the participants were European Americans and most of the remaining $10 \%$ of the participants were African American. Using multivariate regression analysis, Wittenberg and colleagues found that European Americans reported significantly higher preference scores than non-European Americans after controlling for age, gender, and a dummy variable for each dataset (to see if data in a certain dataset might have different results when compared to the others).

Souchek and colleagues (2005) performed a study of adults (with ages from 55 to 80) living in Houston, Texas measuring patients' preferences for mild and severe osteoarthritis with two samples - a public sample and a patient sample. The public sample was comprised of 64 European Americans, 65 African Americans, and 64 Latino Americans who were selected by random-digit dialing. The patient sample was comprised of 66 patients in each of the aforementioned ethnic groups who were selected non-randomly. After combining the two samples, Souchek et al. used rank regression to explore ethnic differences in preference scores controlling for age, gender, and education. African Americans reported significantly higher preference scores for severe osteoarthritis when time trade-off and standard gamble techniques were used. European American scores were not significantly different from Latino American scores. After a brief review of similar literature that found that African Americans reported higher preference scores than European Americans for a variety of disease states, Souchek and colleagues (2005, p. 927) suggest that African Americans may "...hold preservation of life in higher regard than white subjects."

In a 2004 study of 584 racially and ethnically involving diverse pregnant women in the US, Kuppermann and associates found that European American women had higher utilities for babies unaffected by Down syndrome when compared to African American women $(.95$ vs. $.90 ; p<0.001)$ and when compared with Latina and Asian women $(.95$ vs. .91; $<<0.001)$.

Rosen and colleagues (2003) conducted a study with a sample of 62 individuals-one- 
third African American and the remaining two-thirds European American-where the investigators tested risk sensitivity to health states. They found that European Americans were significantly more likely to be risk averse with respective to potential health states when compared to African Americans.

Among a sample of 64 participants awaiting appointments at a pulmonary clinic (most likely in North Carolina) - age 50 to 75-Cykert et al. (1999) measured preference scores for outcomes of lung surgery using standard gamble scenarios. Approximately 80 percent of the sample was European American and the remaining portion was African American. Using nonparametric multivariate regression analyses, African American respondents were found to report significantly higher scores for living with various states of disability as opposed to death when compared to European American respondents. Cykert and colleagues (1999) discussed ethnic differences in trust and religiosity as potential causes for differences in preference scores between European Americans and African Americans.

\section{Discussion}

The findings from the cost utility preference scores literature are mostly in favor of significant differences in preference scores between African Americans and European Americans. These findings are likely to be rooted in the fact that, broadly speaking, from a population viewpoint, African Americans and European Americans possess different body images (psychology and eating disorders literature), temporal orientations (psychology and education literature), and views of disease etiology (medical anthropology literature). African Americans tend to report more satisfaction with their bodies when compared to European Americans (Abrams \& Stormer, 2002; Soh et al., 2006). African Americans report having more of a present temporal orientation while European Americans report having more of a future temporal orientation (Brewster et al., 2007; Halbert et al., 2007). While European Americans are more likely to "medicalize" a disease, African Americans are more likely to "spiritualize" a disease (Abrums, 2000; Toldson \& Toldson, 2001; King et al., 2005). The literature also reports instances where African Americans conceptualize diseases (Roberts et al., 2002) and view of the etiology of disease (Bogart \& Thorburn, 2005) differently from their European American counterparts.

Although these specific concepts were not explicitly stated, other researchers examining preference-based measures anticipate the reasons that are listed in the previous paragraph as reasons for differences in preference-based scores:

It is not immediately apparent why blacks valued health states more highly than the members of the other racial/ethnic groups, though there are a number of potential explanations... It is possible that members of the [different] racial/ethnic groups applied different reference standards when judging health states in the [time trade off] exercise. Their reference standards could have been influenced by personal or cultural beliefs about health, which, in turn, could have been influenced by personal or vicarious experience with illness.... It is [also] 
possible that the time preference of blacks differs from the time preferences of Hispanics and nonHispanic nonblacks (Shaw et al., 2007, p. 486).

The different reference standards correspond to the differences in body image and disease etiology. The different time preferences correspond to the differences temporal orientation.

In summary, a review of the cost-utility literature provides strong empirical evidence that ethnicity or socially constructed race does impact health-related preferences. Preference-scores are consistently significantly different between African Americans and European Americans ethnic groups. Differences in views of the body, temporal orientations, and etiology or conceptualization of diseases each are likely to play a role in these findings.

\section{Racial/Ethnic Differences in Medical Trust}

\section{Social Trust of the Health Care System}

In a study of cancer patients in Rapid City, South Dakota using a sample of 165 patients (52 Native American and 113 non-Hispanic White), Guadagnola and colleagues (2009) examined medical mistrust using multiple linear regression models. They reported the following:

There was a significant difference in mean scale scores for medical mistrust by race... A multivariable regression model including race, age, gender, employment status, annual income, education level, and distance from CCI [Cancer Care Institute] revealed that Native Americans exhibited a significantly higher level of mistrust, even when adjusting for other variables... Native Americans were significantly less likely to trust health care providers, clinics, and hospitals than non-Hispanic Whites (p. 215).

In another study of 196 men newly diagnosed with prostate cancer in the Philadelphia, Pennsylvania metropolitan area (71 African American, 125 European American), Halbert and colleagues $(2009$, p. 2556) utilized regression analysis to examine medical mistrust and found that: "Compared with white men, African-American men reported significantly greater mistrust." In another study of 190 parents (140 African American, 50 European American) at the Primary Care Center at Children's Hospital of Pittsburg, Pennsylvania, Rajakumar et al. (2009) examined parents' attitudes towards medical research. Rajakumar and fellow researchers $(2009$, p. 111) found: "African American parents were 2 times more likely to be distrusting of medical research than white parents after controlling for education level."

Armstrong et al. (2008) examined the relationship of race/ethnicity and social trust in the health care system in a random sample of 236 participants who had been recently seen in by a primary care practice or emergency department in the University of Pennsylvania Health System in the three year prior to the study. There were 144 African 
Americans and 92 European Americans in the study sample. After controlling for multiple variables including sociodemographic characteristics, health insurance/access, and health/psychological status, Armstrong and colleagues found African Americans were not significantly more likely to distrust the health care system than European Americans on issues of competence, but African Americans were significantly more likely to distrust the health care system than European Americans on issues of values.

Smith and colleagues (2007) examined differences in the quality of the patientphysician relationship among terminally ill African American and European American patients. They utilized a cross-sectional survey of 803 patients (115 African American, 688 European American) and two-group comparison of their perceptions on multiple factors. Although they did not find significant differences in the interpersonal trust measure, they found lower ratings of quality among African American patients. Smith and fellow researchers (2007, p. 1581) wrote:

...lower ratings of the patient-physician relationship by African Americans may be a sign of a deeper lack of trust in the health care system to which African Americans have less access than whites despite poorer overall health and that, therefore, can reasonably be perceived as unjust and untrustworthy.

In Chapter 25 of Multicultural Medic ine a nd Health Disparities (2006), Gamble discussed a broad range of areas within the health care system where African Americans report higher distrust than European Americans. African Americans are more likely report lower levels of trust in areas such as organ donation and medical research (2006). However, African Americans were more likely to report higher levels of trust in a study of 118 Baltimore residents (Boulware et al., 2003). Thus, it may be that African Americans may have lower levels of trust in some segments of the health care system, but higher levels of trust in other segments.

Boulware and colleagues (2003) examined racial/ethnic differences in trust among physicians with a sample of 49 African Americans and 69 European Americans, ranging from ages 18 to 75, from the Baltimore, Maryland metropolitan area. The respondents were selected with random digit dialing and had an equal probability of being selected. Using multivariate logistic regression, the researchers controlled for age, gender, education, income, type of insurance, belonging vs. not belonging to an HMO, and prior exposure to medical environments. Results show that African Americans were significantly less likely to trust their physicians although they were more likely to trust their health insurance plan.

In study published in 2000, LaVeist, Nickerson, and Bowie analyzed the connection between race/ethnicity and medical mistrust. Using a sample of 781 African American and 1,003 European American cardiac patients from three hospitals in Maryland, LaVeist and colleagues employed five items from the Medical Mistrust Index to assess similarities or differences in medical mistrust between the two groups using two-sample t-tests. All five items showed a significant difference between African Americans and European Americans. According to LaVeist and colleagues (2000) African Americans 
were:

1) $21 \%$ more likely to believe that hospitals sometimes deceive or mislead patients

2) $63 \%$ more likely to believe that hospitals want to know more than they need to know

3) $95 \%$ more likely to believe that hospitals have sometimes done harmful experiments on patients without their knowledge

4) $91 \%$ more likely to believe that rich patients received better care at hospitals than poor people

5) $57 \%$ more likely to believe that male patients received better care at hospitals than women

These differences found in this study might be considered issues of values as in Armstrong and colleagues' 2008 study.

\section{Interpersonal Trust of Health Care Providers}

In a sample of 1,031 men aged 50 or over (503 African American and 528 European American) from North Carolina and Louisiana, Carpenter and colleagues (2009) used logistic regression to test the association of trust and prostate cancer screenings. They found that African American men reported significantly lower levels of physician trust compared to their European American counterparts. In a telephone survey of 1,681 adults enrolled in Medicare (aged 65 and older) in Allegheny County, Pennsylvania, Musa et al. (2009) used logistic regression techniques and found that African Americans were significantly less likely to: agree that their doctor would fully explain medical research, believe that their doctor would not ask them to participate in harmful medical research, that their physician's care was competent, and have trust in their own physician.

Lee and colleagues (2008) examined the association between patient race/ethnicity and perceived interpersonal aspects of care in the emergency department (ED) using a cross-sectional survey in a sample of 235 European Americans, 108 African Americans, and 29 others. Participants were recruited from a high-volume "tertiary care academic medical center in the southeastern United States" (p. 81). Lee and fellow researchers found: "In multivariate regression, race/ethnicity continued to be a significant predictor of lower trust...after accounting for age, gender, education, household income, health insurance, visits to the ED, clinic and physician in the last six months, and route of referral to the ED" (p. 83).

Armstrong et al. (2007) tested the relationship between race/ethnicity and distrust in physicians. Using 1998-1999 Community Tracking Survey data, Armstrong and colleagues analyzed observations collected via random-digit dialing. There were 7,905 European Americans, 1,529 African Americans, and 1,988 Latino Americans in the 
sample. Their study only included individuals who had a physician or had seen a physician in the past year. Using multivariate Poisson regression, controlling for race/ethnicity, gender, age, income, education level, and insurance coverage, they found that African Americans reported the highest distrust in physicians, followed by Latino Americans and European Americans.

Women of all ethnic groups had lower distrust scores than men of their same ethnic group. For European Americans as age, education, and income increased, distrust decreased. For African Americans and Latino Americans, as education increased, distrust decreased. But there were no significant differences in distrust found for African Americans and Latino Americans of various age and income categories. For all ethnic groups, individuals with no insurance had higher distrust scores than individuals with Medicare, Medicaid, or private insurance. For African Americans and Latino Americans, individuals with private insurance reported lower distrust than African Americans and Latino Americans with Medicare or Medicaid. Armstrong and colleagues also found a good deal of geographic variability in distrust in physicians. For instance, African Americans in Boston, Massachusetts reported higher distrust in physicians than African Americans in Las Vegas, Nevada and Philadelphia.

Halbert and colleagues (2006) explored the relationship between race/ethnicity and trust in physicians using data from the 1999 Kaiser Family Foundation Survey of Race, Ethnicity, and Medical Care. The survey was administered to a nationally representative sample of US households. The sample population included 432 African Americans and 522 European Americans with a mean age of 42.3 years. They controlled for three types of predictor variables: sociodemographic variables, prior health care experiences variables, and structural characteristics of health care variables. They found-using multivariate logistic regression analysis - that African Americans were significantly less likely to report trust in health care providers when compared to European Americans.

Halbert and colleagues (2006, p. 899) stated: "The results of our study suggest that experiences with health care providers and sources of medical care may be more important to trust in health care providers among African Americans than sociodemographics." In other words, the sociodemographic factors used in their logistic regression - gender, educational level, and even race concordance with physician — did not predict levels of trust among African Americans, but experience related factors were significant predictors of trust in health care providers.

Boulware and colleagues (2003) examined racial/ethnic differences in trust among physicians with a sample of 49 African Americans and 69 European Americans, ranging from ages 18 to 75, from the Baltimore, Maryland metropolitan area. The respondents were selected with random digit dialing and had an equal probability of being selected. Using multivariate logistic regression, the researchers controlled for age, gender, education, income, type of insurance, belonging vs. not belonging to an HMO, and prior exposure to medical environments. Results show that African Americans were significantly less likely to trust their physicians although they were more likely to trust their health insurance plan. 
In a study of 909 participants randomly selected for a national telephone survey (527 African American, 382 European American), Corbie-Smith et al. (2002) examined distrust of medical research. The participants were aged 18 years and over. CorbieSmith and researchers (2002) found: "After controlling for other sociodemographic variables in the logistic regression model, race remained strongly associated with a higher distrust score..." (p. 2458).

Doescher and colleagues (2000) explored the relationship between race/ethnicity and trust in the physician using a nationally representative sample of 3,450 African Americans, 1,873 Latino Americans, 27,824 European Americans and 2,187 Americans classified as other. After adjusting for predisposing, enabling, need, and utilization factors from the Anderson-Newman classification, African Americans were significantly less to trust the physician than European Americans. Latino Americans and Americans of other backgrounds also trusted the physician less than European Americans. African Americans reported the lowest amount of trust in the physician.

\section{Discussion}

The literature in this area of interpersonal and social trust is fairly unanimousAfrican Americans report lower levels of trust than their European American counterparts. This is true after sociodemographic characteristics such as income and education are controlled in multivariate analyses. While the body of literature on race/ethnic differences in social trust is weaker due to smaller sample sizes, the body of literature on race/ethnic differences in interpersonal trust is stronger due to larger sample sizes and includes four studies with a nationally representative sample.

In two of the national studies, Latino Americans also reported lower levels of interpersonal trust in health care providers. This suggests that Latino Americans may also view the world of health and health care differently than European Americans. In another study, Native Americans reported higher levels of medical distrust compared to European Americans. Although other racial/ethnic groups' history and culture are not explored in this dissertation, higher levels of distrust among Latino and Native American populations is likely to be rooted in the unique historical injustices that they have faced.

Dovidio and colleagues (2008, p. 484) summarized the literature discussing the continuing influence of past experiences on current medical treatment:

The literature reviewed in this paper provides direct experimental evidence of the impact of racial attitudes and stereotypes on White-Black relations in the United States. In general, although explicit prejudice and stereotypes have declined over time, many Whites still harbor implicit, negative racial attitudes and stereotypes toward Blacks. These implicit biases are manifested in subtle, often unintentional forms of discrimination that produce less favorable outcomes for Blacks than for Whites, contribute to error and miscommunication, and create racial distrust.

Given these findings, we can understand how mistrust persists and perceptions of provider quality are so often divergent between racial/ethnic groups. Again, although the 
focus of this dissertation is on the differences between African Americans and European Americans, the author proposes that such differences are likely to be found wherever two or more racial/ethnic groups have different historical experiences and cultural views that, in turn, impact the ways by which those groups will view the world of health and their society's health care system.

\section{Racial/Ethnic Differences in Perceptions of Provider or System Quality of Care}

There are seven categories of literature in the health services and health disparities literature that discusses perceptions of system or provider quality:

- Satisfaction with the medical encounter

- Time spent with physician

- Wait time to see physician

- Communication, comprehension, and linguistic barriers

- Social distance or race concordance

- Cultural competence

- Perception of bias or discrimination

The distinction between trust and perceptions of provider quality or satisfaction may not yet be clear. Mark Hall (2006, p. 461) makes the difference between the two clear while showing how they are related to the same phenomenon-past experiences. $\mathrm{He}$ wrote:

One definition of interpersonal physician trust that has been proposed is a patient's optimistic acceptance of their vulnerable situation, based on the belief or expectation that the physician will act in the patient's best interests. In contrast, satisfaction is more backward looking, based on past experiences. While satisfaction refers to the patient's opinions of the physician's actions, trust refers to the expectation about the overall relationship with a physician, based in part on experiences with that and other physicians' actions.

Thus, understanding past experiences is crucial in order to understand both trust and perceptions of provider or system quality of care. Past experiences determine the amount of trust a patient will place in their provider before an encounter, while experiences during the encounter determine perceptions of provider or system quality of the encounter that has taken place. 


\section{Perceptions of System Quality of Care}

In the previously discussed study by Guadagnolo and colleagues (2009, p. 220), the researchers found: "Native Americans also expressed lower levels of satisfaction with the health care system than non-Hispanic Whites. These differences in perceptions remain significant even when adjusting for other socioeconomic variables such as annual income, education level, and distance from the cancer care institute."

Fongwa and colleagues (2008) compared 101,189 European American and 8,791 African American Medicare enrollees to compare their perceptions of system quality using multivariate linear regression models. Fongwa and colleagues (2008, p. 1136) found: "Blacks reported significantly worse experiences with getting care quickly, office staff helpfulness, getting needed care, health plan customer service, rating of specialist care, and rating of the health plan... However, they reported better provider communication and rated their personal doctors/nurses and health care more positively."

In a large study analyzing perceived racial discrimination in a sample of 28,519 European Americans, 1,682 Hispanic Americans, and 5,927 African Americans, Hausmann et al. (2008) used multivariable logistic regression to perceived discrimination among whites, African Americans, and Hispanics. They found: "African Americans were more than 3 times as likely to perceive racial discrimination while seeking health care, after controlling for a variety of background characteristics" (p. 910). Hispanics Americans' perceived discrimination did not significantly differ from European Americans.

Using data collected by the Kaiser Family Foundation Survey in 1999, Chen and colleagues (2005) explored the relationship between patients' beliefs about racism, their preference for physician race, and satisfaction with care. With adults eighteen and older, the national sample for the study included 1,479 European Americans, 1,189 African Americans, and 983 Latino Americans. African Americans and Latinos were oversampled. Using analysis of variance (ANOVA), the study investigators found that African Americans reported significantly higher discrimination in health care when compared to European or Latino Americans. In addition, African Americans who preferred an African American physician and had an African American physician were three times more likely to rate their physician as excellent when compared to other African Americans with the same preference for racial concordance but who did not have an African American physician.

In a study of 4,157 randomly sampled participants aged 18 years and older, Blendon and colleagues (2007) examined ratings of the health care system. They examined health care system ratings from 15 different subgroups of 5 different racial/ethnic groups (persons of African, Asian, European, Latin, and Native American descent). African Americans were divided into three subgroups: those born in the US, Africa, and the Caribbean. Latino Americans were divided into four subgroups: Mexican Americans, Central/South Americans, Puerto Rican Americans, and Cuban Americans. Asian Americans were divided into six subgroups: Chinese Americans, Asian Indian 
Americans, Filipino Americans, Korean Americans, Vietnamese Americans, and Japanese Americans. They wrote:

In an ordered logistic regression that controlled for income, education, age, and sex, Native Americans and U.S.-born African Americans were still found to give significantly worse ratings of the health care system than whites. Ratings given by African Americans born in Africa did not differ significantly from those given by whites (p. 1440).

In a review article examining cultural differences in medical communication, Schouten and Meeuwesen (2005, p. 23) identified 14 studies that "involved either audio or video recordings of the consultation or direct scoring of doctor-patient communication during consultations" published between 1974 and 2004. They found: “...[I]t seems safe to conclude that findings do suggest considerable differences and difficulties in communication between doctors and patients from different cultural and ethnic backgrounds" (p. 28).

Ngui and Flores (2005) compared the perceived satisfaction with care and ease of service use for 35,946 parents (28,916 European American, 3,210 Hispanic American, and 3,820 African American) of children with special health care needs across the United States randomly selected. They found that African American and Hispanic American parents reported significantly less satisfaction with care and significantly more problems with each of service use compared to European American parents. After multivariate logistic adjustment, Ngui and Flores found no significant differences between European Americans and Hispanic Americans, but found significant disparities between African Americans and European Americans in ease of service use (but not satisfaction with care).

In a cohort study of 1,072 colorectal cancer patients (80 Asian/Pacific Islander, 103 Hispanic, 85 African American, and 799 European American) in northern California aged 40-84, Ayanian and colleagues (2005) used multivariable linear regression models to evaluate their perceptions of the quality of care for their cancer. After adjusting for multiple variables using linear regression, they found that African American, Asian/Pacific Islander, and Hispanic American parents all found significantly more problems with coordination of care and access to care when compared to European American cancer patients.

Johnson et al. (2004) examined racial/ethnic differences in perceptions of bias and cultural competence in health care. Using a nationally representative sample of participants in the Commonwealth Fund 2001 Health Care Quality Survey, study participants were classified as follows: European Americans $(3,488)$, African Americans (1,037), Latino American (1,153), and Asian. As in the Blendon et al. (2008) study, African Americans were less likely to report communication or comprehension difficulties when compared to Latino and Asian Americans. Unlike the Blendon et al. study, African Americans were not as likely as to report that they had as much time with their doctor as they wanted when compared to Latino and Asian Americans. African 
Americans, Latino Americans, and Asian Americans were significantly more likely to report that they felt they were discriminated on the basis of race/ethnicity and ability to speak English well when compared to European Americans. These findings were reported even when investigators controlled for health literacy, demographic factors, sources of care, self-rated health status, and reports of medical communication.

In a study of 3,406 parents' perceptions of pediatric primary care $(37.9 \%$ Latino, 34.3\% Asian/Pacific Islander, 14.1\% European American, 13.4\% African American, and $0.4 \%$ Native American) among parents of students in a large urban school district in California, Seid and colleagues (2003) examined the following measures by ethnic group: 1) access, 2) continuity, 3) contextual knowledge, 4) communication, 5) comprehensiveness, 6) coordination, and 7) a summary measure of all previous 6 measures. After controlling for education, insurance coverage and type, regular source of care, and chronic condition, researchers found that Asian/Pacific Islanders and Hispanic ethnic groups reported significantly lower satisfaction with communication, access, continuity, and coordination when compared to European Americans. African American parents reported no significant differences except in perceptions of comprehensiveness of care.

\section{Perceptions of Provider Quality of Care}

Using a cross-sectional survey of 915 HIV-infected patients (294 European American, 491 African American, and 130 Hispanic American) in $14 \mathrm{HIV}$ clinics, Korthuis and colleagues (2008) examined patients' perceptions of access and communication from their HIV provider. Using multivariate linear regression to control for sociodemographic variables, the researchers found that African and Hispanic Americans reported significantly longer travel and wait times when compared with European Americans. However, compared to European Americans, African Americans were significantly more likely to report better communication with their provider. They write: "Lurie et al. suggest that higher ratings of patient-provider communication may be the product of lower expectations for healthcare quality among blacks" (p. 2051).

Blendon et al. (2008) explored the perceptions of quality of physician care among fifteen different racial/ethnic subgroups in the US. The randomly selected sample of people (each over seventeen years of age) included 1,001 European Americans, 102 American Indians/Alaska Natives, 1,118 African Americans, 1,045 Latino Americans, and 1,068 Asian Americans. African Americans were divided into three subgroups: those born in the US, Africa, and the Caribbean. Latino Americans were divided into four subgroups: Mexican Americans, Central/South Americans, Puerto Rican Americans, and Cuban Americans. Asian Americans were divided into six subgroups: Chinese Americans, Asian Indian Americans, Filipino Americans, Korean Americans, Vietnamese Americans, and Japanese Americans. Racial/ethnic minorities were oversampled in the study.

Blendon et al. conducted their survey in 2007. Using multivariate logistic regression analyses and controlling for income, age, education, sex, insurance status, nativity, and English proficiency, Blendon and colleagues found that African Americans born in the United States did not report any significant differences in communication or 
comprehension barriers when compared to European Americans. However, African Americans born in the US were significantly less likely to report having regular care with a doctor in the past twelve months and to report that doctors spent enough time with them.

Levinson and colleagues (2008) audiotaped conversations between 89 orthopedic surgeons and their 1007 patients all over the age of 60 in 5 Chicago, Illinois area hospitals in order to assess patient satisfaction with communication by racial/ethnic groups, comparing European and African American patients. Among the surgeons, 85.2\% were European American, 4.5\% were African American, $8 \%$ were Asian/Pacific Islander, and $2.3 \%$ were classified as other. Among the patients, $78.6 \%$ were European American, $19.6 \%$ were African American, and 1.8\% classified as other. Levinson et al. used a variety of statistical tests including logistic regression and found: "In all 11 of the communication ratings, African American patients were significantly less likely to rate the surgeon communication as very good or excellent compared with white patients.... $[T]$ he significance of the relationship between race and satisfaction remained after including a number of potential confounders in the model" (p. 414).

Lee and colleagues (2008) examined the association between patient race/ethnicity and perceived interpersonal aspects of care in the emergency department (ED) using a cross-sectional survey in a sample of 235 European Americans, 108 African Americans, and 29 others. Participants were recruited from a high-volume "tertiary care academic medical center in the southeastern United States" (p. 79). They found no significant differences in patient satisfaction by racial/ethnic group after controlling for other covariates in a multivariate regression.

Mayo et al. (2007) reviewed the existing literature (20 studies) that examined the attitudes and perceptions of Hispanic patients and health care providers. They found the following: "For Hispanic patients, recurrent themes included communication/language barriers, perceptions of care, and differences in care/services" (p. 64). Mayo and colleagues $(2007$, p. 69$)$ also found:

Specifically, providers are limited in interactions with Hispanic patients by communication barriers as well as institutional and resource barriers, such as limited access to interpreters. Providers also report varying levels of comfort with Hispanic patients. Less experienced providers often report greater comfort with Hispanic patients but express concern about communication and cultural differences.

Smith and colleagues (2007) examined differences in the quality of the patientphysician relationship among terminally ill African American and European American patients. They utilized a cross-sectional survey of 803 patients (115 African American, 688 European American) and two-group comparison of their perceptions on multiple factors. African American patients were significantly less likely to agree that their physician: respects the patient, possesses sufficient sensitivity in telling bad news, listens 
to the patient, allows the patient to participate in decisions, and helps the patient with the medical system.

Siminoff and colleagues (2006, p. 355) conducted an observational study using audiotaping "to examine whether patient characteristics are associated with communication patterns between oncologists and breast cancer patients." The study involved 58 oncologists and 405 breast cancer patients (325 European American, 80 nonEuropean American) in two large metropolitan areas in two states. Using logistic regression, they found that physicians spent significantly more time engaged in relationship building with white than non-white patients. They also found that "White patients provided more biomedical information to their physicians than did non-white patients" (p. 357). They concluded:

One recurring difference across most communication categories was race. Racial differences occurred in almost every one of the communication categories examined. White patients had more utterances in almost every communication category than their non-white counterparts. These differences may mean a less adequate decision-making process for patients who are members of racial or ethnic minorities, patients who are less affluent, older, and have less education ( $\mathrm{p}$. $355)$.

Schouten and Meeuwesen (2006, p. 21) reviewed 14 articles examining intercultural medical communication. They wrote:

Findings reveal major differences in doctor-patient communication as a consequence of patients' ethnic backgrounds. Doctors behave less affectively when interacting with minority patients compared to White patients. Ethnic minority patients themselves are also less verbally expressive; they seem to be less assertive and affective during the medical encounter than White patients.

\section{The Effects of Societal Discrimination: Vigilance and Internalized Racism}

Several articles in the literature highlight the multiple ways in which perceived discrimination impacts the health of populations. In a meta-analysis of the perceived discrimination literature by various types (racial, gender, sexual orientation, unequal treatment, other, and not specified), Pascoe and Richman (2009) examined 134 articles discussing the impact of discrimination on health-88 of the articles dealt with racial discrimination. They wrote: "Analysis of 134 samples suggests that when weighting each study's contribution by sample size, perceived discrimination has a significant negative effect on both mental and physical health" (p. 531).

In their review of empirical research, Ahmed, Muhammed, and Williams (2007) discuss how discrimination can impact the health of non-majority groups in three ways: 1) discrimination limits the areas in which minorities can live, 2) discrimination restricts access to health care services and increases the amount of stressors in minorities due to increased rates of unemployment and poverty, and 3) discrimination leads to internalized racism which is related to lower health status among minorities. Other works support this 
line of reasoning (Fiscella \& Williams, 2004; Pietila 2010) and highlight the negative impact of discrimination on the mental health of African Americans (Neighbors et al. 1996; Toldson \& Toldson, 2001; Richman, Kahn-Wood, \& Williams, 2007).

Of special interest, however, is how discrimination plays a role in the use of health services, which might explain why some populations have higher hospitalization rates for ambulatory care sensitive conditions (ACSC) or conditions which can be treated in the outpatient setting. Pascoe and Richman (2009, p. 531) stated: "Perceived discrimination also produces significantly heightened stress responses and is related to participation in unhealthy and nonparticipation in healthy behaviors." Osyerman and colleagues (2007) performed three experiments that provide evidence that discrimination can lead to a heightened vigilance to avoid situations (or places) where someone might feel discriminated. This "prevention-focused vigilance" would help explain why individuals who have an ACSC might avoid seeking treatment with a provider and thus, their health status deteriorates to a point where their condition is urgent or emergent.

Others have highlighted differential responses to racism and discrimination within the African American population. LaVeist, Sellers and Neighbors (2000) found that among African Americans who experienced racism, those with a more system-blaming orientation had a higher survival rate after 13 years than those with a more self-blaming orientation. Their findings indicate that the way African Americans respond to racism influences their health outcomes. This may suggest that African Americans with a more self-blaming orientation may be individuals who internalize racism, while those with a more system-blaming orientation do not internalize racism.

Internalized racism may be associated with lower self-worth and decreased selfesteem (and thereby more externality in health locus of control measures). If this is true, then individuals who internalize racism may be more prone to engage in behaviors that decrease their health status (i.e. sedentary lifestyle, poor dietary habits, not seeing a doctor for an ACSC). In other words, any population that experiences discrimination from the health care sector of their society is less likely to establish a relationship with a primary care provider or work with health care professionals to manage their ACSC, but among the population that experiences discrimination, those who internalize racism will be even worse off than those who do not.

Thrasher and colleagues (2008) discussed evidence illustrating how a system-blaming orientation among African Americans can increase the odds of survival. They hypothesized that higher levels distrust among African Americans with HIV would lead to lower adherence to antiretroviral therapy. They stated:

Our findings seem to indicate the opposite: that negative expectations of their health care providers may increase HIV-infected patients' vigilance about monitoring their care among the minority of patients who are distrustful. Ford and colleagues (in press), for example, found that African American sexually transmitted disease patients who perceived more racism were more likely to get tested for HIV (p. 91). 


\section{Discussion}

The body in this area of the literature is both robust and convincing-African Americans have significantly lower perceptions of provider and system quality when compared to their European American counterparts. Although the literature is not as extensive, Hispanic Americans also report facing multiple challenges in health care system encounters. Multiple studies also found that Americans of Asian/Pacific Islander descent reported significantly lower system and provider quality of care when compared to European Americans. In one study with a large national sample and another study conducted in Pennsylvania, Native Americans were also found to be less likely to rate the system quality of health care as high as European Americans.

Researchers find evidence of lower quality of care in multiple settings including: emergency departments, hospital surgery wards, primary care offices, and HIV clinics. Evidence is also found in the care provided for multiple disease states including breast cancer, colorectal cancer, HIV, and patients who are terminally ill. Overall, there is strong, compelling evidence that several racial/ethnic groups-persons of African, Asian/Pacific Islander, Latin, and Native American descent-experience lower provider and system quality of care when compared to European Americans.

\section{Summary of the Literature Review on the Four Health Cosmology Factors}

The literature in these four areas report significant differences between African Americans and European Americans in the way they view the world of health and health care. The fact that these statistically significant differences emerge after controlling for education and income indicate that there are racial/ethnic differences above and beyond class. It must be emphasized again that although many of the researchers reported racial differences, these differences are not rooted in race as defined by phenotype and genetic distinctions. The differences are rooted in socially assigned race, culture, and historical experiences. Simply put, these are ethnic differences.

With an understanding of these four factors, we can now respond to the question regarding accountability and agency posed by Krieger (2001, p. 672): “...who and what are responsible for population patterns of health, disease, and well-being, as manifested in present, past, and changing social inequalities in health?" Based on the literature reviewed in this chapter, it is clear that social race assignment, culture, and history play a large role in explaining population patterns of health, disease, and wellness. Differences in social race assignment lead to superior medical treatment for some and inferior medical treatment for others. Differences in culture lead to differences in a group's health locus of control, religiosity, religious coping, and preferences for hypothetical disease states. Differences in historical experiences lead to differences in interpersonal and social trust of health care providers and systems, and perceptions of quality in physician and system care. 
Avalon and Young (2005, p. 392) anticipated the manner in which the four ethnic factors presented in this chapter do affect medical utilization or help-seeking behaviors. They wrote:

...[D]espite a well-documented gap in formal help-seeking behaviors between Blacks and Whites, almost no attention has been given to the role of cognitiveaffective variables in explaining these differences. These variables represent beliefs and thoughts about one's experiences in the world. Most researchers have focused primarily on access barriers - such as financial, geographical, or transportation limitations - or on perceived needs, giving little attention to the role that cognitive-affective variables that distinguish between two groups play in creating and maintaining the help-seeking gap. Understanding the role of cognitive-affective variables is especially important, because they are likely due to cultural and social differences that distinguish the two groups. Furthermore, findings can guide the development of educational, outreach, and clinical interventions that meet the specific needs of Blacks.

The author argues that the documented gap in African American and European American views of the world of health and health care, not only affects medical utilization, but health behaviors, and ultimately, health outcomes. These differences in social race assignment, culture, and historical experiences lead to differences in health behaviors and medical utilization that, in turn, determines differences in health outcomes.

Stewart and Napoles-Spring (2003) raised a major counterpoint to the body of literature presented here. They argued that there might be major measurement issues with measures used in health disparities research. Due to major measurement issues, the findings in the body of literature reviewed above may be biased, and in some respects, deeply flawed. While this argument is certainly valid - and the author essentially agrees with Stewart and Napoles-Spring in light of the discussion of race as a variable in Chapter 1 - the measures used to capture the impact of race/ethnicity need not have been precise to document the fact that differences exist, even if many researchers could not fully explain the nature of the differences. The sheer depth and range of the literature from multiple disciplines, demonstrating consistency and robustness in findings, collectively reveals relevant and meaningful differences in the view of the world of health and health care between racial/ethnic groups.

\section{Part III: Review of Methodology Literature}

This dissertation utilized a multi-method quantitative approach to multilevel analysis. Multiple quantitative methods will be used in order to arrive at a broader understanding than possible from utilizing a single method. Multilevel modeling and geographic information science will be used to empirically test the new conceptual framework. Together, the quantitative methodologies can highlight which ethnicity component measures and geographic measures variables significantly predict variation in the dependent variables. 


\section{Review of the Use of Multilevel or Hierarchical Linear Modeling in Health Literature}

Multilevel modeling or hierarchical linear modeling (HLM) has been used in past research to provide better estimates of determinants for health outcomes than single-level ordinary least squares regression analysis. Some research involving multilevel modeling has analyzed asthma in Chicago (Gupta et al., 2008), infant mortality in Shelby County, Tennessee (Williams et al., 2006), neighborhood structure, social processes and health in Chicago (Browning \& Cagney, 2003), and other health outcomes (Pickett \& Pearl, 2001).

Multilevel or hierarchical linear modeling is the appropriate statistical methodology when more than one level of data will be analyzed. Usually, researchers utilize two-level hierarchical linear models. Level I variables include individual characteristics such as demographic variables and health cosmology variables. Level II variables include neighborhood characteristics such as health care facilities availability and developmental toxicants or pollution released in each neighborhood. This approach has been used in past literature to provide a greater understanding of individuals that are nested in particular geographic units.

\section{Review of Geographic Information Science (GIS) Methodology in Health Literature}

GIS approaches have been used in the literature to examine clusters of disease states or risk factors. A few of these articles include studies looking at the geographic clustering of obesity, hypertension, and diabetes in Nashville, Tennessee (Schlundt, Hargreaves, \& McClellan, 2006), the connection between pollution and asthma at the zip code level at a US-Canada border crossing near Buffalo, New York (Oyana and Rivers, 2005). Kouznetsova and colleagues (2007) used negative binomial regression to analyze the connection between diabetes hospitalization and residential proximity to hazardous waste sites in New York City. Ozdenerol and colleagues (2005) used cluster estimation techniques to examine low birth weight clusters in Shelby County, Tennessee. A matched case-control analysis was used to examine the link between air pollution and hospitalization for cardiovascular disease in New Zealand and Australian cities (Barnett et al., 2006).

GIS allows researchers or interested persons in public health to perform three main functions with spatial data according to Cromley and McLafferty (2002): spatial database management, visualization and mapping, and spatial analysis. Spatial data are stored in a database that can be retrieved for later use. The data can then be expressed in the form of a map allowing persons in public health to visualize spatial patterns or clusters of interest. Five various types of analysis can be conducted, including: measurement, topological analysis, network analysis, surface analysis, and spatial statistical analysis (2002).

According to Maheswaran and Craglia (2004, p. 1), GIS has also been used to help public health analysts carry out their mission of "preventing disease, prolonging life, and promoting health through the organized efforts of society." These efforts include: 
1) Disease mapping and spatial analysis

2) Applications in communicable disease control and environmental health protection

3) Applications in healthcare planning and policy

4) Data protection and e-governance issues in public health

In each of these areas there are issues related to the availability of data, the quality of spatial data, the interpretation of hospital admission statistics, the possibility of human error in generating data, the influence of politics in reporting data (2004).

There have also been community-based health initiatives that lead to public participation in the use of GIS (Ozdenerol, 2007). With the concern that GIS technology is inequitably distributed in society, public participation in the use of GIS "enable[s] participants to explore local environmental and social issues, assess their significance, and communicate openly and effectively in attempting to address those issues" (slide 9). Communities can be involved in the creation, evaluation, and analysis of spatial data. As it relates to public health, "GIS adapted by local groups as a tool to raise community awareness of neighborhood conditions and available services, to organize local residents, and ultimately to effect change" (slide 21).

In order for spatial data to be used to study health disparities, several steps must be taken. First, data regarding health outcomes must be obtained.

Health outcome data may be obtained from routine data sources or from specifically collected datasets. Routine data tends to refer to data routinely collected for mainly administrative and management purposes, but which may be used for public health analysis. The main examples are mortality data, hospital admission data, cancer registration data, and congenital malformation data (Maheswaran \& Craglia, 2004, p. 15).

Second, the health outcome data must be linked with foundational spatial data. Foundational spatial data "provide a geographical frame of reference to which other data layers are tied" (Cromley \& McLafferty, 2002, p. 68). In other words, the health outcome data must be linked with a spatial map that allows the data to be referenced with the correct location.

Once the health outcome data are linked with the foundation spatial data, the spatial data analysis can be conducted. According to Maheswaran and Craglia (2004, p. 18), there are two stages of spatial data analysis - exploratory and confirmatory. In the exploratory stage, the analysis involves description and perhaps preliminary hypothesis testing. In the confirmatory stage, empirical models are constructed and hypothesis testing is conducted. GIS, thus, enables "... researchers to locate high prevalence areas 
and populations at risk, identify areas in need of resources, and make decisions on resource allocation" (Ozdenerol, 2007, slide 9).

\section{Food Stores and Food Deserts in the Literature}

GIS has been useful in showing how features of the built environment affect health outcomes. One such example is found in the literature surrounding food stores and food deserts. Food store availability and food deserts have been linked with health outcomes, particularly obesity in the literature. There is a sizeable body of literature on food store availability and food deserts (Moore \& Diez Roux, 2006; Powell et al., 2007; Beaulac et al., 2009). Throughout this literature, African Americans have poorer access to healthy and nutritious food compared to other racial/ethnic groups. As Moore and Diez Rouz (2006, p. 329) stated, "In general, poorer areas and non-White areas also tend to have fewer fruit and vegetable markets, bakeries, specialty stores, and natural food stores."

In their review of the food desert literature published between 1966 and 2007, Beaulac and colleagues (2009) reported that most studies examined food store availability or food deserts with stores per capita measures, while some utilized distance to supermarkets measures. Moore and Diez Rous examined the availability of different types of food stores by census tracts in Forsyth County, North Carolina; Baltimore City and County, Maryland; and New York, New York. Using the same sample of participants found in North Carolina, Maryland, and New York, Moore and colleagues (2008) geocoded participants home addresses and supermarket locations. They used the kernel density method in order give more weight to supermarkets that were closer to participants and less weight to supermarkets which were further away from participants.

\section{Fast Food Restaurants Availability in the Literature}

Fast food restaurant (FFR) availability has also been examined in the literature as a potential predictor of obesity and poor nutrition. Studies have shown that fast food restaurants are more concentrated in African American neighborhoods in New York City (Kwate, 2009) and in poor neighborhoods nationwide (Powell et al., 2007). These studies used FFRs per square mile and FFRs per zip code measures. Another study, using FFR per census tract (Morland et al., 2002), showed fewer FFRs in predominantly African American census tracts compared to predominantly European American census tracts in Mississippi, North Carolina, Maryland and Minnesota. 


\section{CHAPTER 3: METHODOLOGY}

Chapter 3 is divided into two parts. The first part addresses specific aim \#1: construction of the new conceptual framework and explain how populations are situated or nested within neighborhoods and within a cultural and historical context. The second part of this chapter discusses the research design that addresses specific aim \#2. The design of the Jackson Heart Study is reviewed, along with a discussion of sampling techniques, variable operationalization, hypotheses, issues with geographic analyses and data, and the design of the empirical models in this dissertation. Multilevel modeling and geographic information systems will be utilized to analyze the data.

\section{Part I: The $\mathrm{M}^{3}$ Ecosocial Framework-An Ecosocial Theory}

When we combine the four factors discussed in the literature review-views of health stewardship, views of disease and the body, medical trust, and perceptions of medical quality - with the discussion of the social construction of race, we gain a glimpse of how ethnicity in the form of referential, historical, and cultural factors might be linked to health behaviors, medical utilization, and health outcomes. In addition, when we consider the findings of health geographers, spatial epidemiologists, and studies of neighborhoods and health, we move closer to understanding the character of neighborhoods and how the character of neighborhoods might impact individuals.

When these findings, from multiple academic disciplines, are combined with what Nancy Krieger (2001, p. 438) calls "multi-level social epidemiological frameworks," and specifically, with Krieger's ecosocial approach, a new ecosocial conceptual framework emerges - a multilevel, multicultural, and multi-temporal $\left(\mathrm{M}^{3}\right)$ ecosocial framework that explains how neighborhoods, culture, and history affect health outcomes and produce health disparities. This conceptual framework places the previously discussed dominant models in their proper ethnic (historical and cultural) context, while simultaneously situating them in a geographic context to help explain variations in neighborhood health outcomes. This conceptual framework is also informed by the health disparities theories described above. The $\mathrm{M}^{3}$ Ecosocial Framework was the framework from which hypotheses were posed and results were interpreted in this research.

\section{How Ethnicity Impacts Health Behaviors}

The $\mathrm{M}^{3}$ Ecosocial Framework contains three main factors: culture, history, and neighborhoods. This section explains the first two factors-culture and history-in Figure 5. For each individual, the largest circle (ethnicity) represents two referential factors of ethnicity: the socially assigned race and self-reported ethnicity of an individual. The second largest circle (health cosmology) captures four specific ethnic factors that constitute an individual's view of the world of health and health care based on their selfidentification. The smallest circle (individual health behaviors) captures how an 


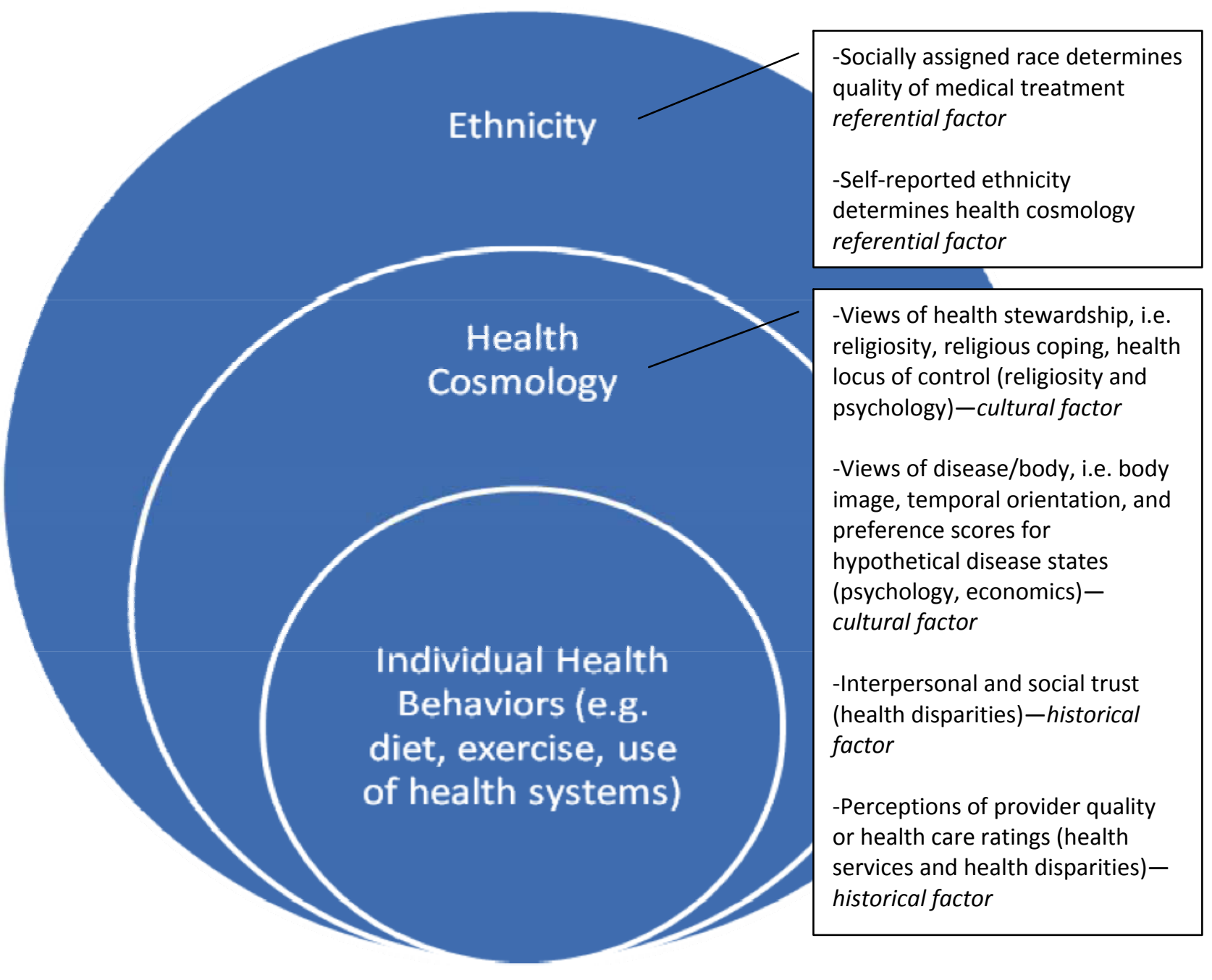

Figure 5: How Culture and History Impact Health Outcomes and Produce Health Disparities 
individual will approach the world of health and health care given their health cosmology.

In this following discussion, more emphasis is placed on the variations in health outcomes between African Americans and European Americans. As stated before, this framework can be used to understand variations in health outcomes for populations around the world. This particular discussion paints a picture of how differences in culture and historical experiences can add to our understanding of health variations in diverse populations concentrating on the differences in African Americans and European Americans.

\section{Ethnicity and the Referential Aspect of Human Behavior}

Throughout recorded human history, human beings have divided themselves into different groups. Usually, this process is based on developing a certain identity or some way of demarcating group boundaries: us vs. them, insider vs. outsider, or emic vs. etic. In the process of doing so, cultural anthropologists tell us that humans use common physical features, learned behaviors, or shared history to define and circumscribe who belongs inside of a certain ethnic group and who belongs outside of an ethnic group (Dressler et al., 2005). How we refer to others and ourselves as human beings constitutes the referential aspect of ethnicity.

Research by Camara Jones and colleagues (2008) illustrate how the referential aspect of ethnicity matters based on socially assigned race. Using data from the Behavioral Risk Factor Social Surveillance, Jones and colleagues found that when people viewed themselves as African American, Native American, or Hispanic American, and people in society viewed them as such, their health outcomes here significantly lower or worse than European Americans. However, when someone viewed himself or herself as African American, Native American, or Hispanic American but people in society viewed them as European American, their health outcomes were not significantly different from people who were socially classified as European Americans.

In other words, this research reveals that how society views a person and classifies them as belonging to one group or another has tremendous implications on the quality of medical treatment one will receive. Socially assigned race determines the quality of treatment based on the meaning of race within a particular society. For example, if a hypothetical society has given preferential treatment to people with blue skin compared to people with green skin, then people with blue skin will receive a higher quality of medical treatment compared to people with green skin. In a society that has been historically stratified by race, the act of identifying the ethnic group to which an individual belongs - the social assignment of race - will be important in determining the quality of medical treatment that individual will receive, as described in the IOM's Unequal Treatment report. Thus, ethnicity plays a role both in the way an individual is classified by people within the society and the way in which the individual self-identifies. 


\section{Health Cosmology}

Because it is a fundamental component of human behavior for human beings to separate and segment themselves into different groups and develop distinctive identities (ways of viewing self and others, i.e., the referential aspect of ethnicity), different groups of people - or various ethnic groups - may possess different health cosmologies - or views of the world of health and health care. Cosmology is defined by the MerriamWebster Online Dictionary (2010) as: "1(a) a branch of metaphysics that deals with the nature of the universe and 1(b) a theory or doctrine describing the natural order of the universe." Cosmology is preferred over psychology due to the fact that: 1) out of the four factors, only health locus of control and religious coping were developed in the field of psychology, and 2) although the Merriam-Webster Online Dictionary defines psychology as "the study of mind and behavior" or "the mental or behavioral characteristics of an individual or a group," the term psychology does not capture the metaphysical element which points to culture nor does it convey the sense of a shared worldview by an ethnic group.

As discussed in the literature review in Chapter 2, African Americans report significantly different responses to several measures that indicate profound differences in the way they view the world of health and health care when compared to European Americans. Sociologist W. E. B. Du Bois (1903) anticipated the development of measures that would allow social scientists to capture the impact of various social phenomena on the health and wellness of human populations within societies over a century ago. Du Bois also anticipated that such measures would reveal different views between those assigned to different races.

There are four main literature-based factors that indicate the aforementioned distinctive views. First, peer-reviewed literature in psychology, religion, and the religion of psychology indicates that African Americans have more of an external health locus of control when compared to European Americans. Literature comparing levels of religiosity or religious coping between African Americans and European Americans, results indicate that African Americans are more religious than European Americans. When combined, these areas suggest that African American views of who is in control of their health and views of religious coping are different from the views of European Americans. Differences in views of health locus of control and religious coping will be classified as cultural determinants.

Second, peer-reviewed literature in economics, specifically in the area of cost-utility analysis, comparisons of racial/ethnic preferences for hypothetical disease states demonstrates significant racial/ethnic differences. Results indicate that African Americans have higher preferences for disease states when compared to European Americans. In other words, European Americans are more risk adverse to negative health conditions when compared to African Americans. This may be due, in part, to an underlying difference in ethnic temporal orientations. Differences in preferences for hypothetical disease states will be classified as cultural determinants. 
Third, peer-reviewed health disparities literature discusses pivotal differences in trust between African Americans and European Americans as it relates to health care. Broadly speaking, African Americans trust health care system less than their European American counterparts. These are differences in social trust. In addition, as it relates measures of interpersonal trust in individual providers, European Americans have higher levels of trust compared to African Americans. An important point to make at this juncture is that the lower levels of interpersonal and social trust among African Americans can be due to the combination of differences in cultural and historical experience (Boulware et al., 2003; Halbert et al., 2006; Kennedy et al., 2007). But since trust is primarily based on one's experience or one's ethnic group's experience, differences in trust will be classified as a historical determinant.

Fourth, peer-reviewed health services literature indicates that African Americans perceive that physicians or health providers give them lower quality of care when compared to European Americans. Whereas measures of trust deal with how an individual looks toward the future based on past an accumulation of one's individual or ethnic group experiences, measures of perception of quality deal with how an individual rates the quality of a specific interaction which occurred in the past. Quality may be measured in terms of time spent with the provider, wait time to see the provider, perceptions of bias or cultural competence, communication or comprehension, or social concordance. In all areas, African Americans report lower levels of quality or satisfaction when compared to European Americans. As above with trust, reporting lower levels of quality can be due to the combination of differences in culture and historical experience (especially discrimination). Since perceptions of quality are primarily based on one's experience or one's ethnic group's experience, differences in perceptions of quality will be classified as a historical determinant.

To reiterate, each of the four factors in an ethnic group's health cosmology are directly linked to Dressler and colleagues' definition of ethnicity. According to Dressler and colleagues' definition of ethnicity, two of the factors can be considered cultural determinants: 1) views of health stewardship, and 2) views of disease and the body. Of course, both of these determinants can be influenced and shaped by historical experiences. But primarily, these factors involve culture (i.e. views of health stewardship involve supernatural beliefs while views of disease and the body also involve supernatural beliefs and temporal orientations). In addition, according Dressler and colleagues' definition and conception of ethnicity as partially defined by common history, two factors can be considered historical determinants: 1) interpersonal and social trust in health care providers and the health care system and 2) perceptions of quality of provider care. Both historical factors are rooted in past experiences.

When viewed together, these four factors demonstrate that there may be an African American Health Cosmology that is qualitatively different from the European American Health Cosmology as evidenced by empirical research in the social sciences. When compared to the European American Health Cosmology, the African American Health Cosmology is characterized by the following: 1) more of an external health locus of control and lower health stewardship, 2) higher preferences for hypothetical disease states 
(due to different body images, time orientations, and disease etiologies), 3) lower levels of trust in the health care system and health care providers, and 4) lower ratings of quality or satisfaction derived from health care interactions.

\section{How Culture Shapes a Population's View of the World of Health and Health Care}

As defined by Dressler et al. (2005, p. 244), culture “...includes shared models for both the mundane (e.g., language use, diet, dress, marriage rituals) and the more abstract (e.g., concepts of self, supernatural beliefs) aspects of life." This means that different ethnic groups have different ways of dealing with the mundane and imagining or conceptualizing the abstract. If we incorporate the insight derived from the ethnomedical approach in medical anthropology, we would conclude that different ethnic groups are characterized by unique ways of dealing with the mundane and conceptualizing the abstract - in ways that shape and inform a population's view of the world of health and health care. This is the fundamental premise of the $\mathrm{M}^{3}$ Conceptual Framework-that different racial/ethnic groups can and do possess different views of the world of health and health care.

This premise draws heavily upon the works of two preeminent scholars-one a sociologist and the other an anthropologist. In 1903, W. E. B. Du Bois, a sociologist, published The Souls of Black Folk . In this text, Du Bois describes a "double consciousness" in the mind of the African American - one American and the other Negro (or African). The concept of the "double consciousness" stated that African Americans had two modes of thinking in one body - one essentially African and the other essentially American or European. In 1941, Melville Herskovits, an anthropologist, published his seminal work The Myth of the Negro Past where he debunked the notion that people of African descent had no cultural legacy when brought to the United States. Herskovits showed that people of African descent in the United States had deep cultural connections to African culture. He termed specific manifestations of these phenomena "Africanisms" and posited that these cultural ties to Africa illustrated a vivid connection between people of African descent in the United States and the cultural legacy of ethnic groups in Africa.

More recently, scholars have advanced the thesis that African American culture is different from European American culture. Toldson and Toldson (2001) postulated the existence of "An African-Centered Psychological Perspective," related to the world of health and health care. James Jones (2003) proposed "TRIOS: A Psychological Theory of the African Legacy in American Culture." According to James Jones, TRIOS constitutes a set of principles involving African culture and represents the foundation an African legacy on the cultural psychology of African Americans. TRIOS, as an acronym for the psychological elements of a cultural system consisting of: time, rhythm, improvisation, orality, and spirituality. These elements are defined as follows:

- Time: personal perspectives on the past, present and future

- Rhythm: patterns of behavior in time, flow, entrainment, movement 
- Improvisation: goal directed creative problem solving under time pressure; a distinctive style

- Orality: preferences for oral face-to-face communication, and personal expression, and the meaningful role of spoken words in human affairs

- Spirituality: belief in the value of a higher power and unknown forces that influence all living things and one's life in particular

The second premise presented here is that there were significant differences in culture before the enslavement of people of African descent in the United States and that these pre-enslavement differences were not wiped away during enslavement. In the national study by Taylor et al. (2007) - discussed earlier in the religiosity and religious coping section of the literature review-the finding that African Americans born in the US were found to be more similar to African Americans born in or migrated from the Caribbean as opposed to European Americans lends support to this proposition - the cultural influence of Africa among people of African descent is enduring. Together, the works of W.E.B. Du Bois and Melville Herskovits provide explanation and evidence that before, during, and after enslavement African Americans possessed a different cultural construct than European Americans. Therefore, some of the differences in health outcomes may be traced back to this particular premise.

When we examine the literature incorporating health locus of control, religiosity and health, and preferences for disease states, it can be argued that in the United States, African Americans and European Americans as ethnic population groups possess different cultural views for their approaches to health and health care. This literature, as discussed in Chapter 2, gives us a clearer understanding of how the behavioral determinant, influenced by and nested in cultural and historical factors, ultimately impacts biology.

\section{How History Shapes a Population's View of the World of Health and Health Care}

According to Dressler et al. (2005, p. 244), a group's "common history (which may include ethnoracial discrimination)" is an important part of defining ethnicity. In the United States, people of African descent experienced slavery, racial segregation, and discrimination resulting in reductions in health and wealth. On the other hand, people of European descent often benefited from slavery, racial segregation, and discrimination resulting in increases in health and wealth (Gaskin, Headen, \& White-Means, 2005). According to Gaskin, Headen, and White-Means (2005), slavery and past discrimination contributed to deficits in wealth among African Americans. This decrease in wealth led to a decreased ability of African Americans in the past to invest in their health and the health of their descendants.

In addition to the history of slavery, racial segregation, and discrimination in a broad sense, African Americans have experienced many specific harmful experiences in the health care system. In Medical Apartheid, Harriet Washington details a laundry list of painful episodes that African Americans endured in the health care system or in the 
pursuit of medical research. These historical experiences lead to differences in interpersonal trust in health providers and social trust in the health care system and also lead to differences in perceptions of provider quality between African Americans and European Americans. Divergent historical experiences can lead to key differences in trust and perceptions of quality and constitutes the third premise of the $\mathrm{M}^{3}$ Ecosocial Framework.

\section{How History Can Affect Beliefs about Disease: The Example of HIV Conspiracy Beliefs}

HIV conspiracy beliefs in the African American community are a classic example of how history can shape an impact an ethnic population's view of a disease. In a nationwide survey of 500 randomly sampled African Americans, approximately 48\% believe that "HIV is a man-made virus", almost 59\% believe that "a lot of information is held back from the public regarding AIDS", and an outstanding 53.4\% believe that there is "a cure from AIDS, but it is being withheld from the poor" (Bogart \& Thorburn 2005, p. 215).

In a clear demonstration of mistrust in the medical community and government, Bogart and Thorburn (2005, p. 215) find that slightly over $16 \%$ of the African American population believes that "AIDS was created by the government to control the black population" and $26.6 \%$ believe that "AIDS was produced in a government laboratory". Although these percentages don't approach a majority, in a population of millions, the numbers add up quickly. This significant minority would be likely to avoid medical care not only for HIV/AIDS, but potentially other diseases as well.

This also indicates how history can shape and impact culture over time. Because of the history of slavery, racial segregation, and discrimination experienced by African Americans, many African Americans adopt what Nancy Boyd-Franklin (2000, p. 13) calls "healthy cultural suspicion"-referring more specifically to psychotherapy, although the principle is the same. While the cultural suspicion is healthy in the sense that many African Americans don't want to be subjected to discrimination, the cultural suspicion is unhealthy in the sense that African Americans may be engaging in more risky sexual behaviors that lead to the contraction of HIV. Bogart and Thorburn found that for African American men, higher HIV conspiracy beliefs were associated with more negative attitudes about condoms. African American men with high HIV conspiracy beliefs did not use condom consistently compared to African American men lower HIV conspiracy beliefs.

Bogart and Thorburn stated that HIV conspiracy beliefs were not prevalent among European Americans in previous research, which is understandable due to the differential experience of European Americans as it relates to slavery, racial segregation, and discrimination. Thus, differential historical experiences can affect the actual beliefs of one ethnic group concerning their view of a disease when compared to another. 


\section{Health Cosmology Summary}

Clearly then, an ethnic group's health cosmology is shaped by cultural components and historical components. Culture and history play vital roles in determining health behaviors, health outcomes, and medical utilization. The fourth premise of the $\mathrm{M}^{3}$ Ecosocial Framework arises from empirical findings in the peer-reviewed literature that demonstrates two different ethnic health cosmologies (broadly speaking) that can be traced to the combination of the different cultures and historical experiences of African American and European American ethnic groups.

\section{The Mutability of Health Cosmology Factors}

It is extremely important to also realize that education, acculturation, and wealth or income can moderate cultural and historical factors. Thus, this conceptual framework of population health is likely to be true for poorer and less educated African Americans when compared to wealthier and more educated African Americans. This also means that these factors are modifiable and not essential to any race or ethnic group - and that because these factors are modifiable, views of the world of health and health care can be changed over time. Because of this, interventions can be developed and policies can be enacted and implemented with the aim of eliminating health disparities. The proposition that an ethnic group's or individual's health cosmology can be changed is the fifth premise of the $\mathrm{M}^{3}$ Ecosocial Framework.

\section{How Neighborhoods Impact Health}

Health cosmology factors impact the health behaviors, medical utilization, and health outcomes of individuals, but they do not do so alone. An individual's ethnicity combines with the individual's neighborhoods to impact health behaviors, medical utilization, and health outcomes. Behaviors can be explained and predicted if we understand the culture and the historical experience of the ethnic group to which an individual belongs in conjunction with the neighborhoods in which the individual lives. The role of ethnicity has been described in Figure 5. Now we will discuss the role of neighborhoods in Figure 6.

The model (Figure 6) illustrates that populations are nested inside of boxes that represent neighborhoods. Inside of those neighborhoods reside populations possessing certain cultures and historical experiences. For any current population, a population's health is not only shaped by their current and past neighborhoods, but their health is also influenced by their ancestors' neighborhoods-the sixth premise of the $\mathrm{M}^{3}$ Ecosocial Framework.

This model depicts time on a diagonal axis to illustrate that both populations and neighborhoods are located on a temporal continuum. In other words, not only are populations nested in a cultural and historical context, but so are neighborhoods. Just as individual humans within a population have life cycles, so do neighborhoods. Neighborhoods reflect the cultures and embody the historical experiences of the 


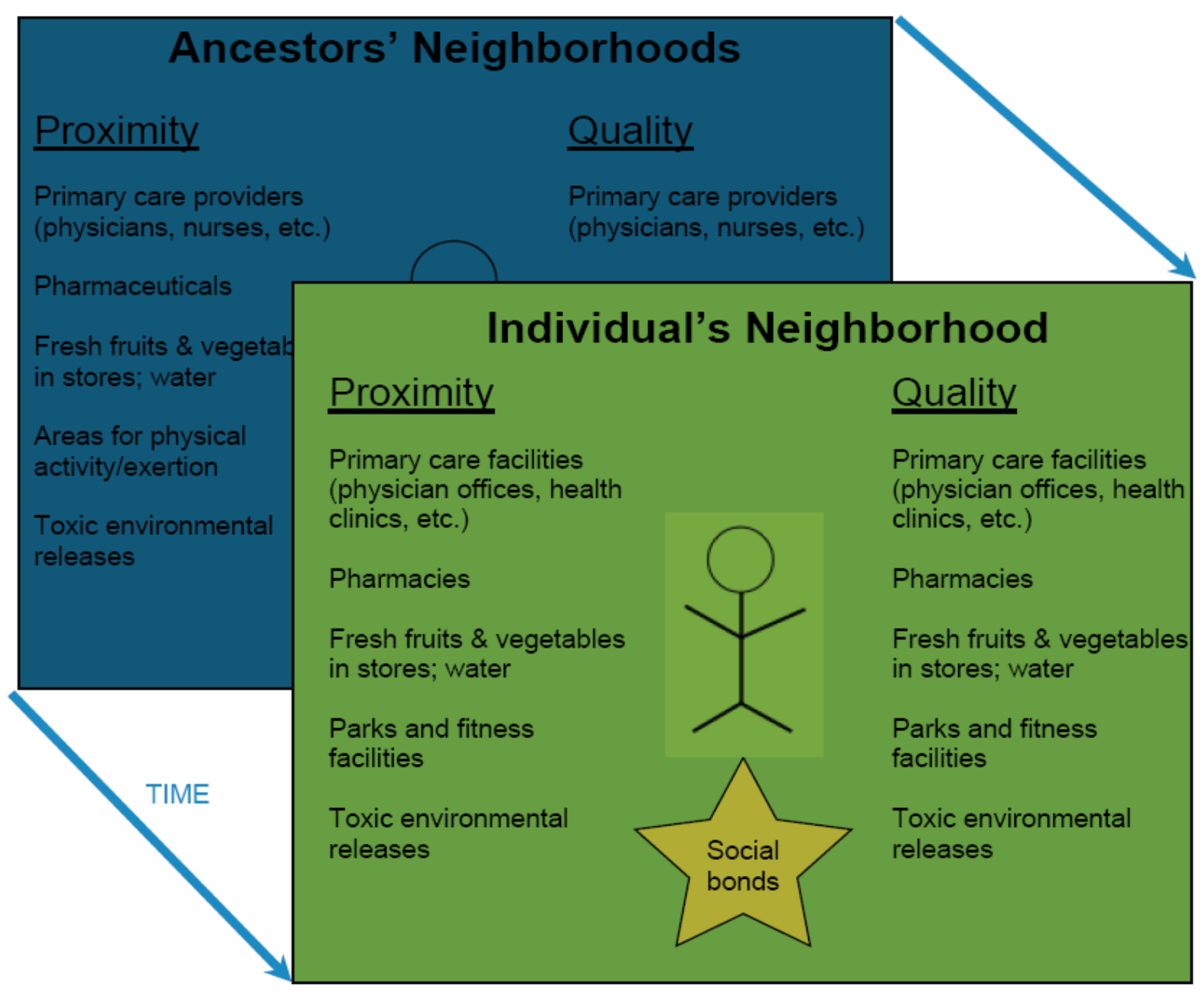

Figure 6: How Neighborhoods Impact Health Outcomes and Produce Health Disparities 
populations that live within them-the seventh premise of the $\mathrm{M}^{3}$ Ecosocial Framework. In any given society that is characterized by historical inequalities along class and ethnic lines, neighborhoods will reflect the levels of power and influence of the population that lives within. Neighborhoods that contain people with more power and influence in a particular society will possess more health-enhancing facilities and fewer health-negating facilities. The converse is true for neighborhoods that contain people with less power and influence in a particular society.

In developed countries, neighborhoods impact the health of an individual in three main ways: via the 1) proximity of health-related facilities, 2) quality of health-related facilities, and 3) the strength of social bonds. For the first two neighborhood factors, an individual's ability to access health-enhancing facilities or be exposed to health-negating facilities is determined by: 1) distance (proximity) and the resulting time it take to reach the good/service or be exposed to toxins and 2) features (qualities) such as hours of availability, level of service, cleanliness, safety, staff friendliness and/or responsiveness, or volume of toxins to which one is exposed. For the third neighborhood factor, the social bonds can moderate or affect the ways by which individuals can access health services or receive attention or care that may be needed.

The green square represents the individual's current and past neighborhoods. The dark blue square represents the aggregation of past neighborhoods of the individual's ancestors. The light blue arrow represents the flow of time from the past to the present. Thus, neighborhoods change over the course of time as human populations migrate and social policies change. The gold star represents the persons in the neighborhood who provide social support for each individual. Social support here can be conceptualized as social cohesion, collective efficacy, social capital, or social networks. Research conducted with each of these concepts highlights the impact social bonds on the health and welfare of individuals within neighborhoods.

Neighborhoods' impact is also weighted by time, or more specifically, cumulative time. In other words, the longer the time one has lived in a neighborhood, the greater the impact of that neighborhood. In addition, the neighborhoods in which an individual has lived have more of an impact than the neighborhoods in which the individual's ancestors lived. The model is essentially a three-dimensional model where time is the z-axis and the map of a neighborhood (represented by the squares) occupies both the $\mathrm{x}$-axis and the y-axis.

\section{Historical Factors and Neighborhood Structure}

Historical factors add a key dimension to our understanding of the way in which social determinants ultimately affect biology via neighborhood structure. In a pluralistic society such as the United States, a nation that is characterized by historical inequalities along class and race lines, neighborhoods are characterized more by the values of those who have political and economic power than those who do not have said power (Pietila 2010). Thus, neighborhoods with a larger concentration of people who possess political and economic power are likely to be characterized by low amounts of toxic environmental 
releases, short proximity to and high quality of health-enhancing facilities, and strong social bonds compared to neighborhoods with a larger concentration of people who do not possess political and economic power within the same society.

Historical factors also explain how the health care system impacts health outcomes. The structure of the health care system is designed on a model (a colonial framework) that does not fit the needs of the African American population, meaning that health care services are not made available in an equitable manner or in a fashion that addresses the needs of the urban African American population (Gish, 1979). The health care system is shaped by the nature and the history of the political economy of primary care in the United States. Health disparities literature indicates that low cultural competency, skin tone biases, and negative stereotypes of nonwhites are serious issues among many health care professionals who serve in the health care system (Smedley et al., 2003; WhiteMeans et al., 2009).

\section{How History, Culture, and Neighborhood Factors Jointly Affect Individual Behaviors}

Ultimately, an individual's health cosmology and neighborhood structure jointly combine to impact and affect an individual's health behaviors, medical utilization, and health outcomes - the eighth premise of the $\mathrm{M}^{3}$ Ecosocial Framework. Due to differences in the way African Americans view the world of health and health care and the differences in the neighborhood structure of many African Americans' neighborhoods when compared to their European American counterparts, African Americans as a whole are less likely to exercise, eat properly, trust in the medical system, and seek modern medical care, while European Americans as a whole are more likely to exercise, eat properly, trust in the medical system, and seek modern medical care when the two populations are compared.

Health behaviors - such as diet and physical activity-are therefore rooted in a geographic, cultural, and historical context. When populations seek medical care, their medical utilization decisions are also based on factors related to geography, culture, and history. This is summed up in Figure 7 - the $\mathrm{M}^{3}$ Ecosocial Framework. The figure shows an individual whose health behaviors, health outcomes, and medical utilization are impacted by both ethnicity and neighborhoods.

Thus, researching the determinants of health outcomes involves much more than the study of genes and microbiological organisms. Understanding the generation of health outcomes and the production of health disparities involves the interdisciplinary study of the social sciences. Altogether, the $\mathrm{M}^{3}$ Ecosocial Framework rests on the following eight premises:

1) Various ethnic groups have different views of the world of health and health care

2) There were pre-existing differences in African and European cultures before the genesis of the Trans-Atlantic Slave Trade 


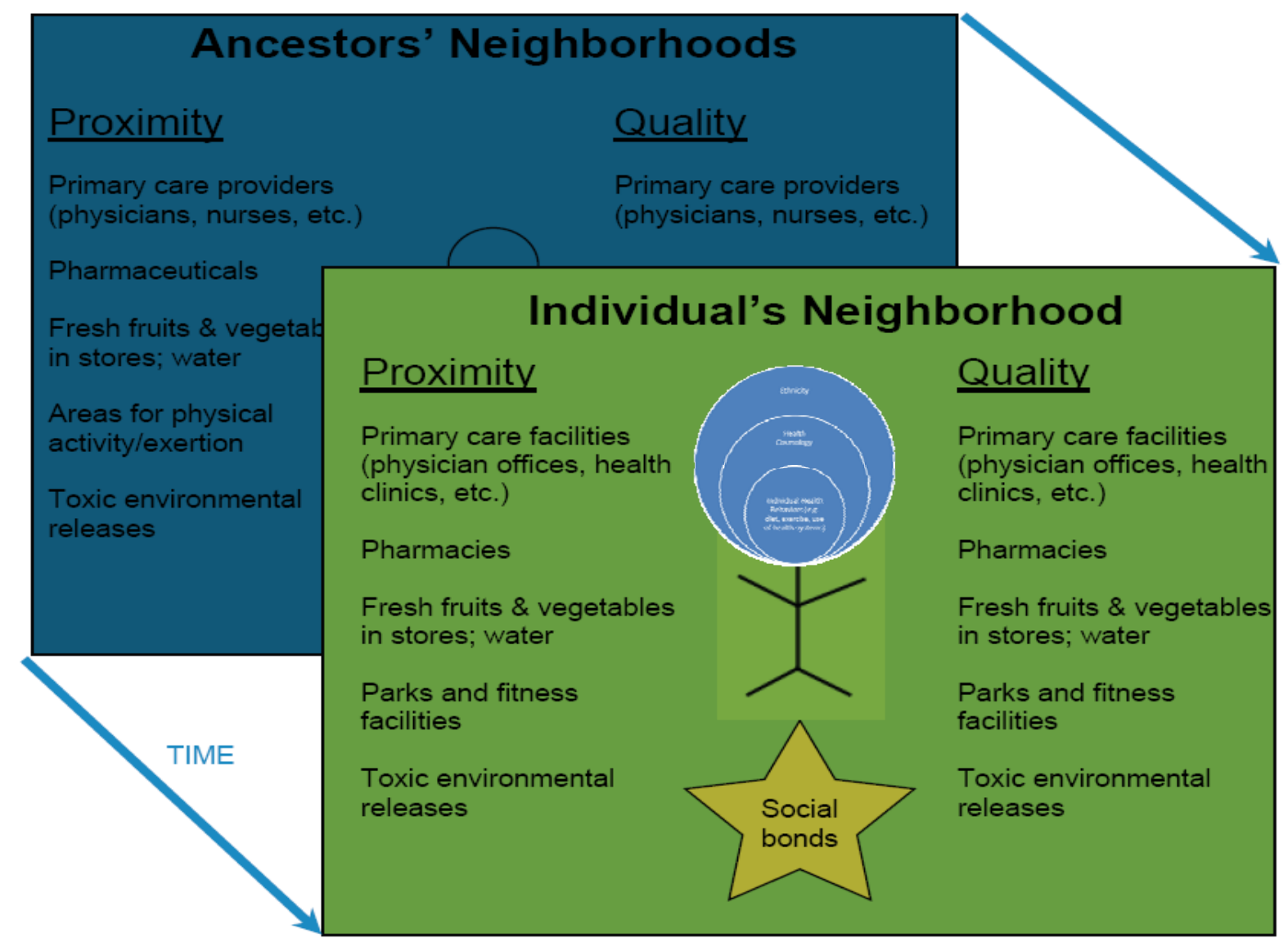

Figure 7: The Multilevel, Multicultural, and Multi-temporal $\left(\mathrm{M}^{3}\right)$ Ecosocial Framework 
3) Divergent historical experiences can lead to key differences in trust and perceptions of quality as it relates to health care

4) Two different ethnic health cosmologies can be traced to the different cultures and historical experiences of African American and European American ethnic groups

5) An ethnic group's or individual's health cosmology can be changed due to education, acculturation, income, wealth, and a new set of experiences

6) For any current population, a population's health is not only shaped by their current and past neighborhoods, but their health is also influenced by their ancestors' neighborhoods

7) Neighborhoods reflect the cultures and embody the historical experiences of the populations that live within them

8) Ultimately, an individual's health cosmology and neighborhood structure jointly combine to impact and affect that individual's health behaviors which in turn impact health outcomes and lead to medical utilization

It is important to reiterate again: The $\mathrm{M}^{3}$ Ecosocial Framework applies to more than African Americans and European Americans in the United States. The $\mathrm{M}^{3}$ Ecosocial Framework draws from the social sciences body of knowledge concerning the cultures and historical experiences of different ethnic groups to explain differences in health outcomes and the existence of health disparities in human populations. The same type of approach described in this chapter can be used to help explain differences in health outcomes and existence of health disparities wherever health disparities exist between two different ethnic groups in a society or nation. Finally, these eight premises are not necessarily unique to the $\mathrm{M}^{3}$ Ecosocial Framework - they represent the synthesis of prior research.

\section{The $\mathbf{M}^{3}$ Ecosocial Framework in Light of Krieger's Ecosocial Theory}

The creation of a new conceptual framework places us in a better position to answer the questions concerning accountability and agency raised by Nancy Krieger (2001, p. 668):

Grappling with notions of causation, in turn, raises not only complex philosophical issues but also, in the case of social epidemiology, issues of accountability and agency: simply invoking abstract notions of "society" and disembodied "genes" will not suffice. Instead, the central question becomes: who and what are responsible for population patterns of health, disease, and wellbeing, as manifested in present, past, and changing social inequalities in health?

The new conceptual framework-developed, discussed, and tested in this dissertation - is also linked to and embedded within the Impact of Race on Health model 
developed by David R. Williams. Combined, the $\mathrm{M}^{3}$ Ecosocial Framework and the Impact of Race on Health model demonstrate the concept of embodiment-according to Krieger (2001, p. 672), "how we literally incorporate, biologically, the material and social world in which we live, from conception to death"-and illustrates the pathways that lead to the embodiment of morbidity and mortality. Finally, the modified Impact of Race on Health model begins to highlight the "cumulative interplay between exposure, susceptibility and resistance" (2001, p. 672) by showing how different environments, neighborhoods, cultures, histories, and identities all combine to affect an individual's exposure to health-producing environments and behaviors and susceptibility to healthnegating environments and behaviors. Based on this information, researchers can now develop more effective ways of resisting health-negating environments and behaviors and amplifying features of health-producing environments and behaviors.

The $\mathrm{M}^{3}$ Ecosocial Framework and the Impact of Race on Health model extend Krieger's Ecosocial Approach by highlighting the historical nature of racial discrimination and ways in which behaviors are rooted in a cultural and historical context. Components of ethnicity are shown to affect the health behaviors of populations and the historical status of populations is shown to shape the structure of neighborhoods.

\section{Part II: Quantitative Research Design}

The $\mathrm{M}^{3}$ Ecosocial Framework explains how six ethnicity factors and six neighborhood factors impact health outcomes and produce health disparities. The six ethnicity factors are: socially assigned race, self-identified ethnicity, views of health stewardship, views of disease/body, interpersonal and social trust, and perceptions of quality or health care ratings. The six neighborhood factors include the proximity and quality of the following: primary care facilities, pharmacies, grocery stores, parks or fitness facilities, toxic environmental release facilities, and social bonds. The $\mathrm{M}^{3}$ Ecosocial Framework and its placement in the Impact of Race on Health model by David R. Williams are depicted in Figure 8 - which will be used to test the proposed conceptual framework.

\section{The Impact of Race on Health Model with the $\mathbf{M}^{3}$ Ecosocial Framework}

The model depicted in Figure 8 has several conceptual and empirical implications. Conceptually, embedding the $\mathrm{M}^{3}$ Ecosocial Framework within the Impact of Race on Health model developed by David R. Williams now allows The Impact of Race on Health model to take into account the nesting of populations within ethnic groups and within neighborhoods. The macrosocial factor biological race is now captured in the "Biological and Geographic Origins" square, while socially assigned race is now captured in the "Ethnicity" circle shown atop the stick figure (representing an individual

person) in the $\mathrm{M}^{3}$ Ecosocial Framework. Thus, biological race is now distinguished from socially assigned race. 


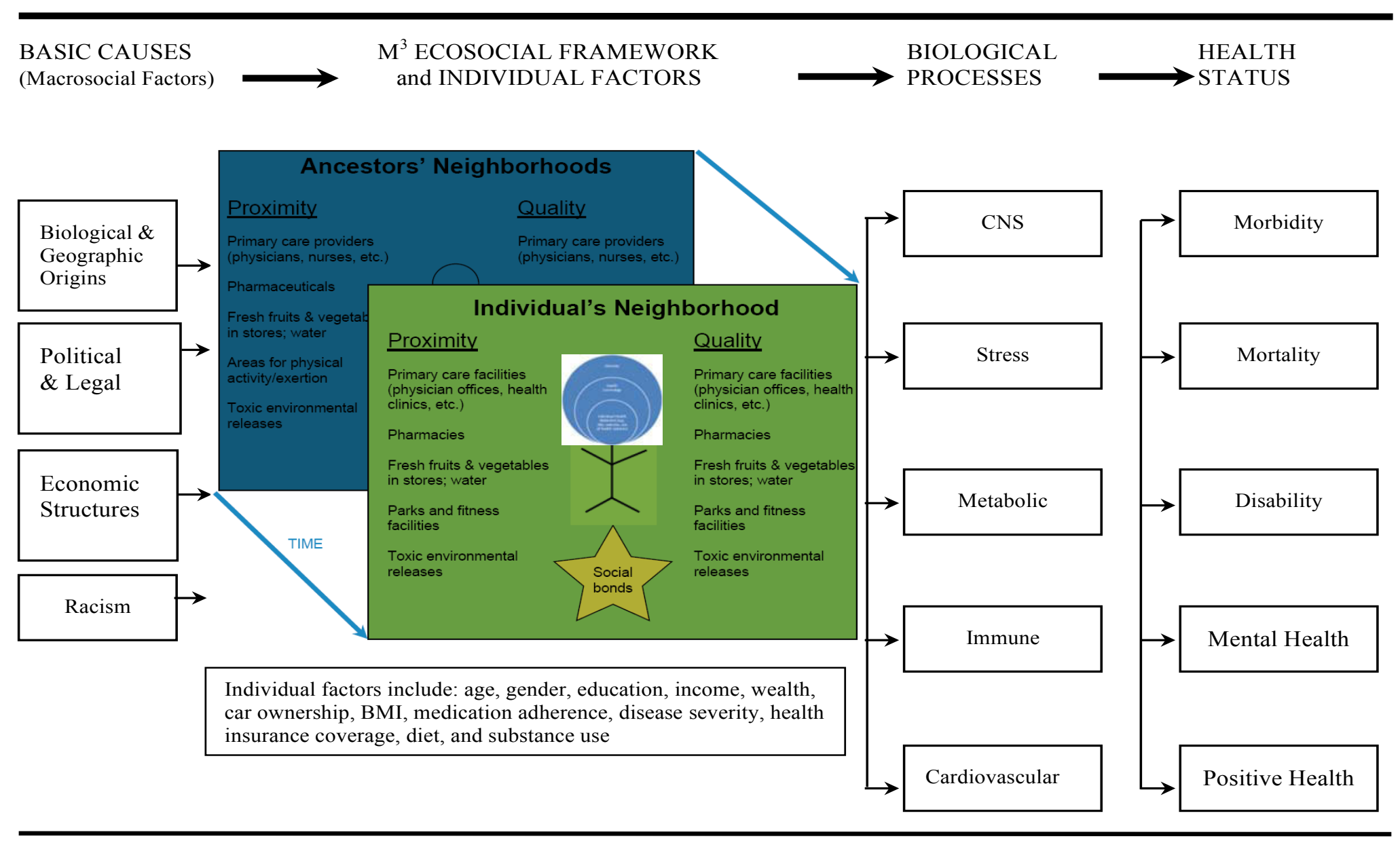

Figure 8: The Impact of Race on Health Model with the $\mathrm{M}^{3}$ Ecosocial Framework

Reprinted from Annals of Epidemiology, Volume 7, Issue 5, David R. Williams. Race and health: Basic questions, emerging directions, pp. 322-33, Copyright (1997), with permission to modify The Impact of Race on Health model from Elsevier. 
Other macrosocial factors in The Impact of Race on Health model, such as economic structures, political and legal frameworks, and the social feature of racism, are to be understood as impacting: 1) the structure of neighborhoods (both historically and contemporarily), 2) the socially assigned race of individuals, and 3) the health cosmology of individuals via historical experiences. This means that, conceptually, researchers cannot discuss neighborhoods, socially assigned race, or health cosmology factors without understanding the macrosocial factors that inform them. While economic structures and political and legal frameworks are often discussed and analyzed in healthrelated research, the social feature of racism is often left out of the discussion, and therefore, cannot be analyzed. But as demonstrated in this dissertation, historical and contemporary racism strongly impacts such a wide range of health-related factors that it becomes exceedingly clear-racism is a fundamental macrosocial factor in explaining health disparities.

The model depicted in Figure 8 also has several empirical implications. Healthrelated researchers can demonstrate how health behaviors and medical utilization - which are both behavioral determinants that impact biological processes and health status (or health outcomes) - are informed by an individual's ethnicity and influenced by an individual's neighborhood structure. Understanding this, health-related researchers using statistical methods can better predict and understand the nature of biological processes and health outcomes now that we have properly contextualized and explained the impact of neighborhoods, culture, and historical experiences.

Altogether, the $\mathrm{M}^{3}$ Ecosocial Framework augments and strengthens the Impact of Race on Health model in the following three ways:

1) Correcting the confounding of race (biology) with ethnicity (socially assigned race and health cosmology)

2) Clarifying how individuals are nested within neighborhoods and ethnicity that jointly impact individual decision-making regarding health behaviors and medical utilization

3) Calling attention to specific features of a neighborhood's structure (or built environment) and how specific neighborhood features will impact health behaviors, medical utilization, and health outcomes

Collectively, these actions will allow health disparities researchers to construct statistical models that produce parameters and results with fewer errors due to confounding and misspecification. Subsequently, more accurate and comprehensive statistical models (and their results) can then lead to the formulation of more effective, evidence-based health policies and interventions. 


\section{Dissertation Hypotheses}

The author hypothesizes that ethnicity and neighborhood factors-discussed in Chapter 2 and earlier in Chapter 3-are significant predictors of physical activity, cardiovascular risk factors (hypertension, diabetes, and hypercholesterolemia), and hospitalizations due to ambulatory care sensitive conditions (including the following: diabetes, hypertension, urinary tract infections, pneumonia, and asthma). Only eight of the 12 factors were tested in this research. Certain measures containing data regarding socially assigned race, pharmacies, and toxic environmental releases were not collected in the data source used in this study. Further, all participants in the data source selfidentified as African American, making self-identification constant. The eight specific hypotheses are outlined in Table 1.

\section{Brief Overview of the Jackson Heart Study}

In order to test the validity of $\mathrm{M}^{3}$ Ecosocial Framework, a large dataset containing multiple measures of culture, history, and neighborhoods was needed. Given the relatively large size of the sample, the scope of the research questions (including a range of sociocultural measures), and the matching purpose of overall study, the author decided to use data collected in the Jackson Heart Study. The Jackson Heart Study (JHS) is a large, community-based, observational study whose participants were recruited from urban and rural areas of the three counties (Hinds, Madison and Rankin) that make up the Jackson, Mississippi metropolitan statistical area (MSA). "The primary objective of the JHS is to investigate the causes of (cardiovascular disease) in African Americans to learn best how to prevent this group of diseases in the future" (Jackson Heart Study, 2003, p. 8). The National Institutes of Health — specifically the National Heart, Lung, and Blood Institute (NHLBI) and the National Center for Minority Health and Health Disparities (NCMHD) - sponsored the study.

\section{Recruitment and Sampling in the Jackson Heart Study}

The Jackson Heart Study recruited participants from four different pools. According to the description of the Jackson Heart Study on the study website (Jackson Heart Study, 2008, website):

Participants were enrolled from...4 recruitment pools: random, 17\%; volunteer, $30 \%$; currently enrolled in the Atherosclerosis Risk in Communities (ARIC) Study, $22 \%$ and secondary family members, $31 \%$. Recruitment was limited to non-institutionalized adult African Americans 35-84 years old, except in the family cohort where those 21 to 34 years of age were eligible. The final cohort of 5,301 participants includes $6.59 \%$ of all African American Jackson MSA residents aged 35-84 ( $\mathrm{N}=76,426$, US Census 2000). 
Table 1: List of Dissertation Hypotheses

\begin{tabular}{|c|c|c|c|}
\hline ID & Hypotheses & Alternative Hypotheses & Key Independent Variables \\
\hline $\mathbf{A}$ & $\begin{array}{l}\text { Health stewardship factors do not } \\
\text { significantly predict health } \\
\text { behaviors, health outcomes, or } \\
\text { medical utilization (culture } \\
\text { hypothesis) }\end{array}$ & $\begin{array}{l}\text { Higher religiosity (in frequency or } \\
\text { intensity) and a higher passive problem } \\
\text { solving score will predict lower physical } \\
\text { activity scores and a higher chance for } \\
\text { disease and ACSC hospitalization }\end{array}$ & $\begin{array}{l}\text {-Church attendance }>1 \mathrm{x} / \text { week } \\
\text {-Prays frequently }>1 \mathrm{x} / \text { week } \\
\text {-Feel God's love for me }>1 \mathrm{x} / \text { day } \\
\text {-Religion helps greatly with stress } \\
\text {-Passive problem solving score }\end{array}$ \\
\hline B & $\begin{array}{l}\text { Views of disease and the body do } \\
\text { not significantly predict health } \\
\text { behaviors, health outcomes, or } \\
\text { medical utilization (culture } \\
\text { hypothesis) }\end{array}$ & $\begin{array}{l}\text { Use of home remedy will predict a } \\
\text { higher chance for disease and ACSC } \\
\text { hospitalization }\end{array}$ & -Ever used home remedy \\
\hline $\mathbf{C}$ & $\begin{array}{l}\text { Interpersonal and social trust do not } \\
\text { significantly predict health } \\
\text { behaviors, health outcomes, or } \\
\text { medical utilization (historical } \\
\text { experience hypothesis) }\end{array}$ & $\begin{array}{l}\text { Greater trust in medical provider will } \\
\text { predict a lower chance for disease and } \\
\text { ACSC hospitalization }\end{array}$ & -Trust in medical provider \\
\hline $\mathbf{D}$ & $\begin{array}{l}\text { Perceptions of provider quality or } \\
\text { health care system ratings do not } \\
\text { significantly predict health } \\
\text { behaviors, health outcomes, or } \\
\text { medical utilization (historical } \\
\text { experience hypothesis) }\end{array}$ & $\begin{array}{l}\text { Greater satisfaction with health care and } \\
\text { no experience with unfair medical } \\
\text { treatment will predict a lower chance } \\
\text { for disease and ACSC hospitalization }\end{array}$ & $\begin{array}{l}\text {-Satisfaction with health care } \\
\text {-Unfair medical experience }\end{array}$ \\
\hline $\mathbf{E}$ & $\begin{array}{l}\text { Proximity to and quality of primary } \\
\text { care facilities does not significantly } \\
\text { predict health behaviors, health } \\
\text { outcomes, or medical utilization } \\
\text { (neighborhood hypothesis) }\end{array}$ & $\begin{array}{l}\text { Greater difficulty reaching health } \\
\text { services will predict a higher chance for } \\
\text { disease and ACSC hospitalization }\end{array}$ & -Difficulty reaching health services \\
\hline
\end{tabular}


Table 1: (cont.)

\begin{tabular}{|c|c|c|c|}
\hline ID & Hypotheses & Alternative Hypotheses & Key Independent Variables \\
\hline $\mathbf{F}$ & $\begin{array}{l}\text { Proximity to and quality of } \\
\text { nutritious, quality food does not } \\
\text { significantly predict health } \\
\text { behaviors, health outcomes, or } \\
\text { medical utilization (neighborhood } \\
\text { hypothesis) }\end{array}$ & $\begin{array}{l}\text { People who report a serious problem } \\
\text { with grocery access and who live in a } \\
\text { census tract in the top quintile of fast } \\
\text { food restaurants will have a higher risk } \\
\text { of disease and ACSC hospitalization }\end{array}$ & $\begin{array}{l}\text {-Serious problem: grocery access } \\
\text {-FF restaurants in top quintile }\end{array}$ \\
\hline G & $\begin{array}{l}\text { Proximity to and quality of parks, } \\
\text { fitness facilities, and built } \\
\text { environment do not significantly } \\
\text { predict health behaviors, health } \\
\text { outcomes, or medical utilization } \\
\text { (neighborhood hypothesis) }\end{array}$ & $\begin{array}{l}\text { People who report a serious problem } \\
\text { with parks, sidewalks, trash/litter, heavy } \\
\text { traffic, and excessive noise will have } \\
\text { significantly lower physical activity } \\
\text { scores and a higher risk of disease and } \\
\text { ACSC hospitalization }\end{array}$ & $\begin{array}{l}\text {-Serious problem: lack of parks } \\
\text {-Serious problem: poor sidewalks } \\
\text {-Serious problem: trash/litter } \\
\text {-Serious problem: heavy traffic } \\
\text {-Serious problem: excessive noise }\end{array}$ \\
\hline $\mathbf{H}$ & $\begin{array}{l}\text { Proximity to and quality of social } \\
\text { bonds do not significantly predict } \\
\text { health behaviors, health outcomes, } \\
\text { or medical utilization (social bonds } \\
\text { hypothesis) }\end{array}$ & $\begin{array}{l}\text {-Low medical transport support will } \\
\text { predict higher risk for disease and } \\
\text { ACSC hospitalization } \\
\text {-Small social network size ( }<.3 \text { friends), } \\
\text { and high stress due to neighborhood } \\
\text { crime will predict lower physical } \\
\text { activity scores and higher risk for } \\
\text { disease and ACSC hospitalization }\end{array}$ & $\begin{array}{l}\text {-Low medical transport support } \\
\text {-Social network size: } 3+\text { friends } \\
\text {-Stress due to neighborhood crime }\end{array}$ \\
\hline
\end{tabular}


Participants were recruited from a mixed family design. Although the sample is not completely random, given the large size of the sample, it is likely that the Jackson Heart Study sample is fairly representative for the target population. However, the Jackson Heart Study sample may be most biased toward participants who trust in medical providers and have a high degree of satisfaction in the health care system.

\section{Study Design in the Jackson Heart Study}

Investigators in the Jackson Heart Study have conducted two major clinical exams with JHS participants and are currently conducting the third exam. The study is further described as follows:

Major components of each exam include medical history, physical examination, blood/urine analyses and interview questions on areas such as: physical activity; stress, coping and spirituality; racism and discrimination; socioeconomic position; and access to health care. At 12-month intervals after the baseline clinic visit (Exam 1), participants are contacted by telephone to: update information; confirm vital statistics; document interim medical events, hospitalizations, and functional status; and obtain additional sociocultural information. Questions about medical events, symptoms of cardiovascular disease and functional status are repeated annually. Ongoing cohort surveillance includes abstraction of medical records and death certificates for relevant International Classification of Diseases (ICD) codes and adjudication of nonfatal events and deaths. (Jackson Heart Study, 2008, website).

The baseline clinical visits took place between 2000 and 2004. Approximately three years after the baseline clinic visit, participants return to the clinic for Exam 2. In this dissertation, measures for all independent variables and two dependent variables (total physical activity score and hypercholesterolemia) were collected during the baseline clinic visit. Data for the three remaining dependent variable were based on data collected in Exam 2 (diabetes and hypertension status) or on hospitalizations which took place after Exam 1 (ACSC hospitalizations).

The original goal for the author in this dissertation was to conduct an analysis of all dependent variables as measured in Exam 2 (or afterwards in the case of ACSC hospitalizations). However, measures from some variables were not collected during Exam 2 (total physical activity score) or were not available in the same format as Exam 1 measures (hypercholesterolemia). This explains why some measures were tested using Exam 1 measures while others were tested using Exam 2 measures.

Multiple clinical visits by participants in the JHS were justified from an epidemiological perspective. The study was designed in order "to investigate the causes of CVD in African-Americans to learn how to best prevent this group of diseases in the future" (JHS Protocol, Manual 1). By measuring multiple variables during the baseline visit, JHS researchers could then analyze which variables might statistically predict the occurrence of various CVD risk factors and outcomes. Additionally, causation cannot be 
determined in a cross-sectional analysis. Therefore, a prospective design was a necessity in order to help determine how variables measured during Exam 1 might affect outcomes measured after Exam 1-including measures taken during Exam 2 or any time after Exam 1 (i.e. hospitalization or CVD mortality).

JHS investigators also obtained blood pressure measures, measured height and weight, and collected urine and blood samples (for lipid, hemostasis, hematology, and chemistry measurements). Genetic information was also obtained from a subsample of study participants to conduct genetic epidemiology analyses. Study design information has been previously published (Sempos et al., 1999; Payne et al., 2005; Taylor et al., 2005). More information regarding the Jackson Heart Study and its design can be found in the JHS Protocol, Manual 1 that is available online.

\section{Variable Operationalization}

\section{Dependent Variables}

Ethnicity and neighborhood contextual measures - as independent variables-were tested (while controlling for covariates) to see if there is a significant relationship with the following dependent variables:

- Total physical activity score- - health behavior measure

- Hypertension as a CVD risk factor (as defined by sitting blood pressure) health outcome measure

- Diabetes as a CVD risk factor (as defined by hemoglobin A1c levels) - health outcome measure

- Hypercholesterolemia as a CVD risk factor (as defined by JHS algorithm)health outcome measure

- Ambulatory care sensitive condition hospitalization (as defined by the Agency for Healthcare Research \& Quality) — medical utilization measure

\section{Independent Variables}

The independent variables in this section were collected at the individual level (or collected from each individual). The variables in this section are all Level 1 variables in the multilevel statistical analyses. Thus, these variables can be classified as Level 1 or individual level variables.

No data were collected regarding socially assigned race among participants in the Jackson Heart Study. All participants self-identified as African American. Thus, neither of the referential factors variables was included in the analysis since one was missing and 
the other is constant. The following variables are based on the four health cosmology factors found in Figure 5:

- Views of health stewardship - church attendance frequency, prayer frequency, spirituality intensity (Feel God's love), religious helps cope with stress, passive problem solving

- Views of disease/body — folk medicine (use of home remedies)

- Interpersonal and social trust - trust in health care provider

- Perceptions of provider quality—satisfaction with health care, discrimination (experienced unfair medical treatment)

The following variables were used to test for the impact of the proximity and quality of neighborhood facilities in conjunction with the impact of social bonds on health. Although these variables deal with neighborhoods, they were collected at the individual level. These variables are based on the neighborhood factors in Figure 6:

- Proximity and quality of primary care facilities - difficulty in accessing health services

- Proximity and quality of fresh fruits and vegetables—-lack of access to adequate food and/or shopping (i.e. grocery access)

- Proximity and quality of parks and fitness facilities - lack of parks and playgrounds, status of sidewalks, excessive noise, heavy traffic, trash/litter

- Social bonds - medical transport support, social network size, stress due to neighborhood crime

Other general neighborhood perception variables such as excessive noise, heavy traffic or speeding cars, and trash/litter are considered factors that might affect physical activity levels. These are conceptualized as perceptions of built environment factors.

The following traditional explanatory variables were used as covariates. This list of covariates is based on Ansari's meta-analysis (Ansari, 2007) of variables that have been tested before and reported in the literature:

- Demographics—age, gender

- Socioeconomic status-education, individual income/net worth

- Pre-existing CVD risk factor-diabetes, hypertension, hypercholesterolemia

- Lifestyle risk factors - smoking status, alcohol consumption, physical activity 
- Anthropometric measures-body mass index (BMI)

- Use of home remedies (proxy for self-reported adherence to medication)

- Previous myocardial infarction (proxy for severity of illness)

- Health insurance coverage

\section{Neighborhood Characteristics}

The independent variables in this section were collected at neighborhood level (or collected from each neighborhood). The variables in this section are all Level 2 variables in the multilevel statistical analyses. Thus, these variables can be classified as Level 2 or neighborhood level variables.

Census tracts were used as proxies for neighborhoods. Census tract measures included: percentage of African Americans, percentage of people with a college degree (bachelors), percentage of people with a high school diploma, and the median annual household income. Each of these measures was obtained from the 2000 US Census.

The author considered using residential segregation as a measure in the empirical model. However, given the small size of the census tracts, measuring residential segregation using the amount of land occupied or owned by each race becomes problematic. Thus, the author decided to use the percentage of African Americans in each census tract to measure the proportion of the total population that is African American. In essence, this measure serves as a proxy of residential segregation. The hypothesis here is that the larger the percentage of African Americans in a census tract, the lower the political power of residents within that particular census tract due to the prevailing impact and influence of racism.

The fast food restaurant availability measure was constructed using ArcGIS 9.2 (Environmental Systems Research Institute, 2006) and OpenGeoDa (Anselin, 2009). The author constructed the measure in a manner different from the ways discussed in the articles covered in the "fast food restaurant availability in the literature" section at the end of Chapter 2. A 10-mile radius from the center of the census tract was used for two reasons. First, only JHS investigators were allowed access to participants' addresses in order to geocode each participant's address. Second, a 10-mile radius from the center of the census tract was preferred to using the number of fast food restaurants within a census tract. A 10-mile radius would more accurately reflect those fast food restaurants that would be readily accessible to most participants. It is entirely conceivable that a resident living on the edge of a census tract might be only a mile or two away from fast food restaurants located in an adjacent census tract.

The following measures were considered as literature-based due to their use as traditional explanatory variables in past research involving multilevel analyses: percentage of African Americans, percentage of people with a college degree (bachelors), percentage of people with a high school diploma, and the median annual household 
income. The fast food restaurant availability measure was considered as a factor testing a component of the $\mathrm{M}^{3}$ Ecosocial Framework: as a proxy for the availability of fresh fruits and vegetables. Although the fast food availability measure has been tested in past research, the measure has not been conceptualized in the manner depicted in Figures 6, 7, and 8 -where fast food availability is considered as one among several specific neighborhood factors that impact health behaviors and health outcomes.

A list of all variables used in the statistical analyses is found in Table 2.

\section{Statistical Methodology}

Descriptive statistics were used to summarize the distribution of baseline participant characteristics (means for continuous variables and percentages for categorical variables). Multilevel statistical methods were used to account for participants' nesting in neighborhoods. Two-level hierarchical linear models were used to account for the clustering of participants within neighborhoods. The two levels of the model were: the individual-level (Level 1) and the neighborhood-level (Level 2). In this dissertation, census tracts served as proxies for neighborhoods and served as the Level 2 unit of analysis.

A multi-level model (or hierarchical linear model) is defined by specifying a distinct model at each level within the hierarchical structure. Typically, the model at a given level will resemble a "standard" linear model such as linear or logistic regression. The models are placed in a hierarchy by modeling parameters at lower levels as "outcomes" at higher levels. Therefore, these models examine relationships between variables within a given level and specify the relations of variables at one level with that of variables at another. Clustering is accounted for by allowing the coefficient to vary randomly across the higher levels (i.e., individual and neighborhood).

Both SAS 9.1 (SAS Institute, 2004) and PASW Statistics 18 (SPSS Inc., 2009) were used to conduct the statistical analyses. SAS was used to conduct the main statistical analysis, while PASW/SPSS was used to conduct recoding and clean the dataset. PASW/SPSS was also used for deriving the descriptive statistics, conducting the means test of the descriptive statistics, and for double-checking results in SAS. The parameters were estimated in SAS using Proc Glimmix, Proc Logistic, and Proc Reg and in PASW/SPSS using the Genlin procedure. The two-level HLM logit models were designed to help determine if ethnicity and neighborhood structure are predictors of health behavior (physical activity), biological processes (diabetes, hypertension, hypercholesterolemia), and medical utilization due to morbidity (ACSC hospitalization) while controlling for key covariates.

In order to test the impact of ethnicity and neighborhoods measures on physical activity, diabetes, hypertension, and hypercholesterolemia, a two-step analysis was conducted. In the first step, participants in the overall study sample were included in the 
Table 2: List of Variables for Statistical Models

\begin{tabular}{|c|c|c|c|}
\hline ID & Variable & Description of Variable (Measure Description) & Measure Construction \\
\hline \multicolumn{4}{|c|}{ Dependent Variables } \\
\hline 1 & PAT01 & Total physical activity score-exam 1 & $\begin{array}{l}\text { Score based on } 4 \text { domains of activity: work, } \\
\text { active living, sports, home/yard activities }\end{array}$ \\
\hline 2 & HTN217 & $\begin{array}{l}\text { Hypertension status - exam } 2(0=\text { not hypertensive; } \\
1=\text { hypertensive })\end{array}$ & $\begin{array}{l}\text { Clinical visit; blood pressure }>140 / 90 \mathrm{mmHg} \\
\text { (per JNC 7) and/or use of hypertensive meds }\end{array}$ \\
\hline 3 & DMA2104 & $\begin{array}{l}\text { Diabetes status-exam } 2(0=\text { not diabetic; } 1= \\
\text { diabetic })\end{array}$ & $\begin{array}{l}\text { Clinical visit; Fasting glucose } 126+\mathrm{mg} / \mathrm{dl} \\
\text { (per ADA 2004) or use of anti-diabetic meds }\end{array}$ \\
\hline 4 & HCL0103 & $\begin{array}{l}\text { Hypercholesterolemia_exam } 1(0=\text { no high } \\
\text { cholesterol; } 1=\text { high cholesterol })\end{array}$ & $\begin{array}{l}\text { Clinic visit; High total cholesterol }(>240+ \\
\mathrm{mg} / \mathrm{dL}) \text {, high LDL cholesterol }(160+\mathrm{mg} / \mathrm{dL}) \text {, } \\
\text { or use of cholesterol lowering medications }\end{array}$ \\
\hline 5 & ACSC_Hosp & $\begin{array}{l}\text { Hospitalization due to diabetes, hypertension, } \\
\text { pneumonia, asthma, or urinary tract infection }(0=\text { no } \\
\text { ACSC hospitalization; } 1=\text { ACSC hospitalization })\end{array}$ & $\begin{array}{l}\text { Hospital abstracts from Jackson area } \\
\text { hospitals; ACSC categories derived from } \\
\text { Agency for Healthcare Research and Quality }\end{array}$ \\
\hline \multicolumn{4}{|c|}{ Literature-Based Covariates } \\
\hline 6 & Age & Continuous measure from $20-95$ & Home interview; self-reported \\
\hline 7 & Gender & $0=$ female $; 1=$ male & Home interview; self-reported \\
\hline 8 & Edudummy & $0=$ no college degree $; 1=$ college degree & Home interview; self-reported \\
\hline 9 & $\mathrm{INC} 01$ & $\begin{array}{l}\text { Income in categories adjusted by family size; } \\
\text { categories range from } 1 \text { to } 9 \text { where } 1=\text { impoverished } \\
\text { and } 9=\text { relatively wealthy }\end{array}$ & Home interview; self-reported \\
\hline 10 & PDSA26x & Personal wealth level $(0<\$ 200 \mathrm{k} ; 1=\$ 200 \mathrm{k}+)$ & Home interview; self-reported \\
\hline 11 & PDSA25y & Number of cars owned $(0=$ none; $1=1+)$ & Home interview; self-reported \\
\hline 12 & SMK01 & $\begin{array}{l}\text { Smoking status }(0=\text { never smoked; } 1=\text { past } / \text { current } \\
\text { smoker })\end{array}$ & Home interview; self-reported \\
\hline 13 & ALC01 & Alcohol consumption $(0=$ no; $1=$ yes $)$ & Home interview; self-reported \\
\hline 14 & PAT01 & $\begin{array}{l}\text { Total physical activity score (scores range from } 3.0 \\
\text { [low]-16.85 [high]); high scores indicate higher } \\
\text { activity }\end{array}$ & Home interview; self-reported \\
\hline 15 & BMI01 & Body mass index (ranges observed from 14.63-91) & Home interview; self-reported \\
\hline 16 & MSRA43Ax & Used other home remedies ever $(0=$ no; $1=$ yes $)$ & Clinical visit; self reported \\
\hline
\end{tabular}


Table 2: (cont.)

\begin{tabular}{|c|c|c|c|}
\hline ID & Variable & Description of Variable (Measure Description) & Measure Construction \\
\hline 17 & XMI01 & $\begin{array}{l}\text { Past myocardial infarction hospitalization }(0=\text { no; } 1 \\
=\text { yes })\end{array}$ & $\begin{array}{l}\text { Clinical visit; self-reported then record } \\
\text { pulled }\end{array}$ \\
\hline 18 & INS01 & $\begin{array}{l}\text { Health insurance status ( } 0=\text { uninsured; } 1=\text { any } \\
\text { insurance) }\end{array}$ & Home interview; self-reported \\
\hline \multicolumn{4}{|c|}{ Culture Variables } \\
\hline 19 & RCPA1X & $\begin{array}{l}\text { Organized religiosity: church attendance }(0< \\
1 \mathrm{x} / \text { week } 11=1 \mathrm{x} / \text { week }+)\end{array}$ & Home interview; self-reported \\
\hline 20 & RCPA2Y & $\begin{array}{l}\text { Private religiosity: prayer frequency }(0<1 \mathrm{x} / \text { day; } 1= \\
\text { everyday }+)\end{array}$ & Home interview; self-reported \\
\hline 21 & RCPA3EX & $\begin{array}{l}\text { Feel God's love for me frequency }(0<\text { everyday; } 1= \\
\text { everyday }+)\end{array}$ & Home interview; self-reported \\
\hline 22 & RCPA4x & $\begin{array}{l}\text { Religion helps cope with stress }(0<\text { very involved; } 1 \\
=\text { very involved) }\end{array}$ & Home interview; self-reported \\
\hline 23 & PFD01 & $\begin{array}{l}\text { Problem focused disengagement (passive problem } \\
\text { solving score); scores range from 4-20; higher scores } \\
\text { indicate a high degree of passive problem solving }\end{array}$ & Home interview; self-reported \\
\hline \multicolumn{4}{|c|}{ Historical Experience Variables } \\
\hline 24 & TRS01PR & $\begin{array}{l}\text { Trust in healthcare provider }(0=\text { little/no trust; } 1= \\
\text { somewhat/very much })\end{array}$ & Home interview; self-reported \\
\hline 25 & SAT01HC & $\begin{array}{l}\text { Satisfaction with physician }(0=\text { little/no satisfaction; } \\
1=\text { somewhat } / \text { very satisfied })\end{array}$ & Home interview; self-reported \\
\hline 26 & DISA9Ax & Unfair medical treatment $(0=$ no; $1=$ yes $)$ & Home interview; self-reported \\
\hline \multicolumn{4}{|c|}{ Social Support or Social Network Variables } \\
\hline 27 & ISLA6x & $\begin{array}{l}\text { Trouble finding someone to take me to the doctor? } \\
(0=\text { no; } 1=\text { yes })\end{array}$ & Home interview; self-reported \\
\hline 28 & SOCA5Y & Social network size $(0<3$ friends; $1=3+$ friends $)$ & Home interview; self-reported \\
\hline \multicolumn{4}{|c|}{ Individual Perceptions of Neighborhood Problems } \\
\hline 29 & AF3B24x & $\begin{array}{l}\text { Excessive noise }(0=\text { minor/no problem; } 1=\text { serious } \\
\text { problem })\end{array}$ & Home interview; self-reported \\
\hline
\end{tabular}


Table 2: (cont.)

\begin{tabular}{|c|c|c|c|}
\hline ID & Variable & Description of Variable (Measure Description) & Measure Construction \\
\hline 30 & AF3B25x & $\begin{array}{l}\text { Heavy traffic or speeding cars }(0=\text { minor } / \text { no } \\
\text { problem; } 1=\text { serious problem })\end{array}$ & Home interview; self-reported \\
\hline 31 & AF3B26x & $\begin{array}{l}\text { Lack of access to adequate food and/or shopping }(0= \\
\text { minor/no problem; } 1=\text { serious problem })\end{array}$ & Home interview; self-reported \\
\hline 32 & AF3B27x & $\begin{array}{l}\text { Lack of parks/playgrounds }(0=\text { minor/no problem; } 1 \\
=\text { serious problem })\end{array}$ & Home interview; self-reported \\
\hline 33 & AF3B28x & $\begin{array}{l}\text { Trash/litter }(0=\text { minor } / \text { no problem; } 1=\text { serious } \\
\text { problem })\end{array}$ & Home interview; self-reported \\
\hline 34 & AF3B29x & $\begin{array}{l}\text { No sidewalks or poorly maintained sidewalks }(0= \\
\text { minor/no problem; } 1=\text { serious problem })\end{array}$ & Home interview; self-reported \\
\hline 35 & HCA01x & Difficulty getting health services $(0=$ easy; $1=$ hard $)$ & Home interview; self-reported \\
\hline 36 & WSIA30x & $\begin{array}{l}\text { Stress from neighborhood crime }(0=\text { low/no stress; } 1 \\
=\text { high stress })\end{array}$ & Home interview; self-reported \\
\hline \multicolumn{4}{|c|}{ Literature-Based Census Tract Level Neighborhood Factors (used for multilevel modeling) } \\
\hline 37 & C_over80AA & $\begin{array}{l}\text { Participant lives in census tract in top quintile of } \\
\text { percentage of African Americans }(0=\text { no; } 1=\text { yes })\end{array}$ & US Census Bureau 2000 \\
\hline 38 & Medinc 80 & $\begin{array}{l}\text { Participant lives in census tract in top quintile of } \\
\text { median income [above } \$ 50,600](0=\text { no; } 1=\text { yes })\end{array}$ & US Census Bureau 2000 \\
\hline 39 & C_over80dip & $\begin{array}{l}\text { Participant lives in census tract with }>80 \% \text { residents } \\
\text { with high school diploma }(0=\text { no; } 1=\text { yes })\end{array}$ & US Census Bureau 2000 \\
\hline 40 & C_over20deg & $\begin{array}{l}\text { Participant lives in census tract with }>20 \% \text { residents } \\
\text { with high school diploma }(0=\text { no; } 1=\text { yes })\end{array}$ & US Census Bureau 2000 \\
\hline \multicolumn{4}{|c|}{ Specific Neighborhood Factor (used for multilevel modeling) } \\
\hline 41 & FFR10m_INT & $\begin{array}{l}\text { Participant lives in census tract with } 220+\text { fast food } \\
\text { restaurants within a } 10 \text { mile radius from the center of } \\
\text { the census tract }(0=\text { no; } 1=\text { yes })\end{array}$ & $\begin{array}{l}\text { Mississippi State Department of Health and } \\
\text { list developed by Technomics Inc. (a food } \\
\text { industry market research company) }\end{array}$ \\
\hline \multicolumn{4}{|c|}{ Identification Variables } \\
\hline 42 & SUBJID & Participant's ID number & \\
\hline 43 & CensusTract & Participant's census tract & \\
\hline
\end{tabular}


analysis to test factors that might predict whether or not a person will be diagnosed with diabetes or hypertension in Exam 2 or hypercholesterolemia in Exam 1. In the second step, the same analysis was conducted only including participants who live in the least advantaged census tracts (LACTs) to test if certain predictors might influence health for participants living in disadvantaged neighborhoods.

This approach may lead to knowledge about how individuals can behave or how disadvantaged neighborhoods can plan to improve health outcomes. This strategy allowed the author to test for the factors that significantly impact the dependent variables in both the overall study sample and in a smaller sample where persons are more at risk for disease and poor health behaviors - in the least advantaged census tracts. For the last dependent variable (ACSC hospitalizations), all participants were included in the analysis. There were not enough ACSC hospitalizations to allow an analysis to be conducted with only participants living in the least advantaged census tracts.

The dependent variable physical activity is a continuous variable; therefore a hierarchical linear model with a normal distribution will be used to analyze this dependent variable. The following four dependent variables are each binomial categorical variables:

1) Hypertension status (hypertension or no hypertension)

2) Diabetes status (diabetes or no diabetes)

3) Hypercholesterolemia (high blood cholesterol or no high blood cholesterol)

4) Hospitalization due to an ambulatory care sensitive condition (ACSC hospitalization or non-ACSC hospitalization, including no hospitalization)

Therefore, these four dependent variables were tested using a hierarchical linear model specifying a binomial distribution and logit link.

\section{GIS Methodology}

In this dissertation, GIS is used to visualize and explore fast food restaurant availability characterizing potential access. GIS provides a tool for viewing geographical variation in accessibility and seeing if differences stem from gaps in service coverage or are structured across class, ethnic, or racial lines. The maps as output of this dissertation reveal the differential patterning of accessibility.

\section{Specific Models for Each Dependent Variable}

The hierarchical linear model allows us to determine the coefficients or predictors for both individual level characteristics (i.e. ethnicity, perceptions of community needs, race, address, etc.) and neighborhood level characteristics (e.g. percentage of the total 
population that is African American). The specific models for each of the five dependent covariates were constructed as follows:

\section{Physical Activity Score Model}

- Level 1 (total physical activity score) $=$ age + gender + college degree + income category + personal wealth $>\$ 200,000+$ car ownership + past or current smoker + consumed alcohol in past year + BMI + prior heart attack + church attendance $>1 \mathrm{x} /$ week + prays frequently $>1 \mathrm{x} /$ day + feel God's love $>$ $1 \mathrm{x} /$ day + religion helps to deal with stress + passive problem solving + stress due to neighborhood crime + social network size + serious problem: parks and playgrounds + serious problem: excessive noise + serious problem: heavy traffic + serious problem: trash/litter + serious problem: poor sidewalks (22 variables)

- $\quad$ Level 2 (total physical activity score) $=\%$ with high school degree $+\%$ with bachelors degree + median annual household income $+\%$ African American out of total population (4 variables)

\section{Hypertension and Diabetes Status Models (Both Models Are the Same)}

- $\quad$ Level 1 (hypertension or diabetes status $)=$ age + gender + college degree + income category + personal wealth $>\$ 200,000+$ car ownership + past or current smoker + consumed alcohol in past year + physical activity score + $\mathrm{BMI}+$ health insurance coverage + folk medicine (home remedy use) + prior heart attack + church attendance $>1 \mathrm{x} /$ week + prays frequently $>1 \mathrm{x} /$ day + feel God's love $>1 \mathrm{x} /$ day + passive problem solving + trust in medical provider + satisfaction with health care + unfair medical treatment + difficulty to reach health services + social network size + stress due to neighborhood crime + low medical transport support + serious problem: excessive noise + serious problem: heavy traffic + serious problem: grocery access + serious problem: parks/playgrounds + serious problem: trash/litter + serious problem: poor sidewalks (30 variables)

- $\quad$ Level 2 (hypertension or diabetes status) $=\%$ with high school degree $+\%$ with bachelors degree + median annual household income $+\%$ African American out of total population + number of fast food restaurants in census tract (5 variables)

\section{Hypercholesterolemia Status Model}

- Level 1 (hypercholesterolemia status) = same as hypertension and diabetes models except for the following two variables + diabetes status (exam 1) + hypertension status (exam 1)

- Level 2 (hypercholesterolemia status) $=\%$ with high school degree $+\%$ with bachelors degree + median annual household income $+\%$ African American 
out of total population + number of fast food restaurants in census tract ( 5 variables)

\section{Ambulatory Care Sensitive Condition (ACSC) Hospitalization Model}

- Level 1 (ACSC hospitalization $)=$ age + gender + college degree + income category + personal wealth $>\$ 200,000+$ car ownership + past or current smoker + consumed alcohol in past year + physical activity score + BMI + health insurance coverage + folk medicine (home remedy use) + prior heart attack + church attendance $>1 \mathrm{x} /$ week + prays frequently $>1 \mathrm{x} /$ day + feel God's love $>1 \mathrm{x} /$ day + religion helps to deal with stress + passive problem solving + trust in medical provider + unfair medical treatment + difficulty to reach health services + social network size + stress due to neighborhood crime + low medical transport support + serious problem: excessive noise + serious problem: heavy traffic + serious problem: grocery access + serious problem: parks/playgrounds + serious problem: trash/litter + serious problem: poor sidewalks (31 variables)

- Level 2 (ACSC hospitalization $)=\%$ with high school degree $+\%$ with bachelors degree + median annual household income $+\%$ African American out of total population + number of fast food restaurants in census tract $(5$ variables)

The physical activity model is the same as the health outcomes models with the exclusion of medical-related variables and food-related variables which theoretically have no impact on determining a person's physical activity score or their level of physical activity. Of course, the physical activity score variable is omitted as an independent variable because it is the dependent variable. These are the other variables omitted from the physical activity model:

- Health insurance coverage

- Folk medicine (home remedy use)

- Trust in medical provider

- Satisfaction with health care

- Unfair medical treatment

- Difficulty reaching health services

- Low medical transport support

- Serious problem: grocery access

- Number of fast food restaurants in a census tract 
For the least advantaged census tract (LACT) analyses, the following statistical techniques were used for each dependent variable with only variables listed in Level 1 equations above (since census tract level measures were already controlled in the selection of the LACTs):

- Physical activity: ordinary least squares regression analysis (Proc Regression)

- Hypertension status: logistic regression analysis (Proc Logistic)

- Diabetes status: logistic regression analysis (Proc Logistic)

- Hypercholesterolemia status: logistic regression analysis (Proc Logistic) 


\section{CHAPTER 4: RESULTS AND ANALYSIS}

\section{Introduction}

Chapter 4 begins with a description of the sample of Jackson Heart Study participants. Next, the results of each statistical model are presented for each of the dependent variables: physical activity, hypertension status, diabetes status, hypercholesterolemia, and hospitalization due to ambulatory care sensitive conditions. Following each results section is an analysis of the results relative to the $\mathrm{M}^{3}$ Ecosocial Framework. Finally, a summary of the decision rules based on the dissertation hypotheses are discussed along with limitations of the dissertation research methodology.

\section{Descriptive Statistics}

Table 3 gives an overview of the participants in the Jackson Heart Study. The sample is divided into two categories: residence in a least advantaged census tract (those who live in one and those who do not) and gender (females and males). The least advantaged census tracts (LACTs) are defined as census tracts that have (relative to the census tracts within the study sample) more than the median of percentage of African Americans in a census tract (greater than or equal to $93.32 \%$ ) and by census tracts in the bottom 2 quintiles of percentage of people with a high school diploma (less than or equal to $67.753 \%$ ) and the median annual household income (less than or equal to $\$ 28,333$ ). Table 3 includes measures that are used in the statistical analyses (except for Hemoglobin A1c and cerebrovascular disease). Several maps of the study area for the Jackson Heart Study are located in the Appendix.

The "independent-samples $t$ tests" procedure in PASW/SPSS was used to determine if there were significant differences in the measures collected between gender groups and between people living in LACTs and those who do not. Significance levels were set at the 0.05 level and the 0.01 level. This analysis was conducted in order to describe the study sample and illustrate where there might be significant differences in the populations in particular categories.

\section{Description of JHS Sample}

The JHS sample is characterized by stunning health outcomes and stark contrasts. Nearly two-thirds of the sample was classified as hypertensive during Exam 1. By Exam 2 , seven out of ten participants living in more advantaged census tracts were diagnosed with hypertension, while a remarkable eight out of ten participants living in least advantaged census tracts were diagnosed with hypertension. In the time between Exam 1 and Exam 2, the incidence of diabetes increased at least ten percentage points among participants in all four categories. In Exam 1, body mass index statistics reveal that, on average, participant were obese in every category except among males. 
Table 3: Descriptive Statistics of the Jackson Heart Study

\begin{tabular}{|c|c|c|c|c|}
\hline Variable Not & $\begin{array}{c}\text { LACTs } \\
\mathbf{( 3 8 6 5 )}\end{array}$ & $\begin{array}{c}\text { LACTs } \\
(1370)\end{array}$ & $\begin{array}{c}\text { Female } \\
(3364)\end{array}$ & $\begin{array}{c}\text { Male } \\
(1942) \\
\end{array}$ \\
\hline \multicolumn{5}{|c|}{ Literature-Based Covariates } \\
\hline Total physical activity score (\%) & $8.551 * *$ & $7.637 * *$ & $8.149^{* *}$ & $8.610^{* *}$ \\
\hline Hypertension status: exam $1(\%)$ & $59.00^{* *}$ & $73.00^{* *}$ & $64.00^{*}$ & $60.00 *$ \\
\hline Hypertension status: exam 2 (\%) & $70.00^{* *}$ & $81.00^{* *}$ & $76.00^{* *}$ & $68.00^{* *}$ \\
\hline Diabetes status: exam $1(\%)$ & $17.00^{* *}$ & $24.00^{* *}$ & $20.00 *$ & $17.00^{*}$ \\
\hline Diabetes status: exam 2 (\%) & $27.00^{* *}$ & $38.00^{* *}$ & $31.00 *$ & $27.00 *$ \\
\hline Hypercholesterolemia: exam 1 (\%) & $32.00^{*}$ & $36.00^{*}$ & 33.00 & 34.00 \\
\hline ACSC hospitalization, 2000-2009 & 2.00 & 3.00 & 2.00 & 2.00 \\
\hline Cerebrovascular disease: exam 1 (\%) & $5.00 * *$ & $6.00 * *$ & 4.00 & 5.00 \\
\hline Hemoglobin A1c (mean) & $6.085^{* *}$ & $6.259^{* *}$ & 6.097 & 6.066 \\
\hline Age at time of exam 1 (mean) & $54.87^{* *}$ & $59.53^{* *}$ & 55.33 & 54.06 \\
\hline College degree (\%) & $66.01 * *$ & $47.90^{* *}$ & 61.44 & 61.14 \\
\hline Income category (mean) & $2.73 * *$ & $2.36^{* *}$ & $2.61 * *$ & $2.94 * *$ \\
\hline Personal wealth $=\$ 200,000+(\%)$ & $8.97 * *$ & $4.26^{* *}$ & $5.90 * *$ & $10.94 * *$ \\
\hline Car ownership (\%) & $93.73 * *$ & $89.28 * *$ & $92.66^{* *}$ & $95.57^{* *}$ \\
\hline Past or current smoker (\%) & $32.20^{* *}$ & $39.90^{* *}$ & $26.00^{* *}$ & $44.34 * *$ \\
\hline Consumed alcohol in past year (\%) & $46.00^{* *}$ & $41.00 * *$ & $38.00^{* *}$ & $59.00 * *$ \\
\hline Body mass index: exam 1 (mean) & 31.63 & 32.07 & $32.84 * *$ & $29.86^{* *}$ \\
\hline Body mass index: exam 2 (mean) & 32.00 & 32.13 & $32.94^{* *}$ & $30.29 * *$ \\
\hline Past heart attack (\%) & $6.00^{* *}$ & $8.00^{* *}$ & $5.00^{* *}$ & $7.00^{* *}$ \\
\hline Health insurance coverage (\%) & $87.00 *$ & $84.00 *$ & 87.00 & 86.00 \\
\hline \multicolumn{5}{|c|}{$\mathbf{M}^{3}$ Ecosocial Framework Variables } \\
\hline Church attendance $>1 \mathrm{x} /$ week $(\%)$ & 79.17 & 81.27 & $84.46^{* *}$ & $70.97 * *$ \\
\hline Prays frequently > 1x/day $(\%)$ & 81.17 & 82.98 & $86.01 * *$ & $72.41^{* *}$ \\
\hline Feel God's love for me > 1x/day (\%) & 79.15 & 79.81 & $81.68^{* *}$ & $74.57 * *$ \\
\hline Religion helps greatly with stress (\%) & 64.68 & 63.37 & $67.76^{* *}$ & $58.54 * *$ \\
\hline Passive problem solving score (\%) & $76.21^{* *}$ & $77.72 * *$ & $79.79 * *$ & $69.83 * *$ \\
\hline Used home remedy in past 2 wks (\%) & 2.57 & 2.07 & $2.95^{*}$ & $1.93 *$ \\
\hline Ever used home remedy (\%) & 48.15 & 46.07 & 48.42 & 47.78 \\
\hline Trust in medical provider $(\%)$ & 96.00 & 97.00 & $97.00^{*}$ & $95.00 *$ \\
\hline Satisfaction with health care (\%) & 96.00 & 96.00 & 96.00 & 96.00 \\
\hline Unfair medical treatment (\%) & 13.32 & 14.65 & $15.58 * *$ & $10.64 * *$ \\
\hline Difficulty reaching health services (\%) & 11.77 & 12.61 & $13.18^{* *}$ & $9.33 * *$ \\
\hline
\end{tabular}


Table 3: (cont.)

\begin{tabular}{|c|c|c|c|c|}
\hline Variable & $\begin{array}{c}\text { Not LACTs } \\
(\mathbf{3 8 6 5 )}\end{array}$ & $\begin{array}{c}\text { LACTs } \\
(1370)\end{array}$ & $\begin{array}{c}\text { Female } \\
(3364)\end{array}$ & $\begin{array}{c}\text { Male } \\
(1942)\end{array}$ \\
\hline Stress due to neighborhood crime $(\%)$ & $19.79 * *$ & $32.19 * *$ & 23.01 & 22.93 \\
\hline Low medical transport support (\%) & $9.37 * *$ & $12.93 * *$ & 13.18 & 8.41 \\
\hline Social network size: $3+$ friends $(\%)$ & 46.65 & 47.05 & 47.27 & 45.37 \\
\hline Serious problem: excessive noise $(\%)$ & $13.92 * *$ & $18.87 * *$ & 14.03 & 13.75 \\
\hline Serious problem: heavy traffic $(\%)$ & $24.79 * *$ & $31.94 * *$ & 25.42 & 23.72 \\
\hline Serious problem: grocery access $(\%)$ & $8.19 *$ & $10.77 *$ & 8.38 & 7.86 \\
\hline Serious problem: lack of parks $(\%)$ & $13.19 *$ & $17.06^{*}$ & 14.95 & 12.84 \\
\hline Serious problem: trash/litter (\%) & $15.81 * *$ & $22.72 * *$ & $17.17^{*}$ & $13.37 *$ \\
\hline Serious problem: poor sidewalks (\%) & $17.93 *$ & $21.40^{*}$ & 18.88 & 16.21 \\
\hline Combined neighborhood issues (mean) & $.945 * *$ & $1.226 * *$ & $.983 *$ & $.880 *$ \\
\hline \multicolumn{5}{|c|}{ Census Tract Level Variables } \\
\hline Census tract over $\$ 50,600$ MAHI (\%) & $21.00 * *$ & $0.00 * *$ & $19.00 *$ & $23.00^{*}$ \\
\hline Census tract $>20 \%$ Bachelor deg $(\%)$ & $37.38 * *$ & $0.88 * *$ & $35.83^{*}$ & $40.06^{*}$ \\
\hline Census tract $>80 \%$ HS diploma $(\%)$ & $29.81 * *$ & $0.00 * *$ & $28.21 *$ & $32.60 *$ \\
\hline Census tract: top quintile black/AA (\%) & $68.14 * *$ & $100.00 * *$ & $69.11 *$ & $66.46^{*}$ \\
\hline FF restaurants in top quintile (\%) & $24.00 * *$ & $74.00 * *$ & $39.00 *$ & $35.00 *$ \\
\hline
\end{tabular}


By Exam 2, participants were obese, on average, in every category. These results reveal a study population that is at high-risk for cardiovascular disease at the point in time that Exam 1 is conducted. In addition, a higher percentage of participants exhibited more risk factors for cardiovascular disease by the time Exam 2 is conducted.

In several categories, females and people living in the least advantaged census tracts were significantly worse off compared to males and people living in the more advantaged census tracts. Females and people living in least advantaged census tracts had significantly lower total physical activity scores, income levels, personal wealth, car ownership rates, and rates of persons with college degrees. Thus, it is not surprising to see that females and people living in least advantaged census tracts had higher percentages of participants diagnosed with diabetes and hypertension compared to men and people living in more advantaged census tracts.

Of interest among the cultural variables, females reported significantly higher religiosity compared to males in the sample. Over seven out of ten people in the sample attend church more than once a week indicating the strong role of faith and religion in the African American population in Jackson, Mississippi. Among historical experience variables, females reported significantly greater amounts of unfair medical treatment experiences and difficulty reaching health services when compared to males.

Of interest among the neighborhood variables, nearly two out of every ten persons in the study are highly stressed due to neighborhood crime. In the least advantaged census tracts, however, over three out of ten persons are highly stressed due to crime. A significantly higher percentage of persons living in the least advantaged census tracts report serious problems with the neighborhood factors compared to people living in the more advantaged census tracts. While approximately one-third of all study participants lived in census tracts in the top quintile of fast food restaurant availability, nearly threefourths of participants living in the least advantaged census tracts were living in census tracts in the top quintile of fast food restaurant availability.

\section{Results}

\section{Physical Activity (Exam 1)}

\section{Physical Activity in Overall Study Sample (Exam 1)}

This analysis was conducted in SAS using Proc Glimmix to control for Level 1 and Level 2 variables. The results for the total physical activity score in exam 1 for the overall study sample are in Table 4. The model's generalized chi-square/df $=5.12$ indicating a degree of overdispersion. The generalized chi-square statistic generated from a multilevel model is a measure of goodness of fit. According to Douglas Luke (2005, pp. 57-58), such a statistic measures: 
Table 4: Results for Physical Activity Score in Exam 1 in Overall Study Sample

\begin{tabular}{|c|c|c|c|}
\hline Variable & B (Slope) & Std. Error & p-Value \\
\hline \multicolumn{4}{|c|}{ Literature-Based Covariates } \\
\hline Age at time of exam $1^{*}$ & -0.065 & 0.006 & $<.0001$ \\
\hline Male gender* & 0.407 & 0.135 & 0.027 \\
\hline College degree* & 0.409 & 0.156 & 0.009 \\
\hline Income category* & 0.259 & 0.072 & .0003 \\
\hline Personal wealth $=\$ 200,000+$ & 0.193 & 0.218 & 0.377 \\
\hline Car ownership & 0.512 & 0.302 & 0.090 \\
\hline Past or current smoker & 0.186 & 0.140 & 0.184 \\
\hline Consumed alcohol in past year & -0.008 & 0.132 & 0.954 \\
\hline Body mass index* & -0.037 & 0.008 & $<.0001$ \\
\hline Past heart attack* & -0.706 & 0.334 & 0.035 \\
\hline \multicolumn{4}{|c|}{$M^{3}$ Ecosocial Framework Variables } \\
\hline Church attendance $>1 \mathrm{x} /$ week & 0.316 & 0.168 & 0.060 \\
\hline Prays frequently $>1 \mathrm{x} /$ day & 0.132 & 0.172 & 0.445 \\
\hline Feel God's love for me $>1 \mathrm{x} /$ day & -0.014 & 0.159 & 0.928 \\
\hline Passive problem solving score & -0.011 & 0.022 & 0.606 \\
\hline Stress due to neighborhood crime & 0.252 & 0.152 & 0.098 \\
\hline Social network size: $3+$ friends* & 0.250 & 0.122 & 0.040 \\
\hline Serious problem: excessive noise & -0.070 & 0.215 & 0.743 \\
\hline Serious problem: heavy traffic & -0.031 & 0.168 & 0.856 \\
\hline Serious problem: lack of parks & -0.074 & 0.194 & 0.702 \\
\hline Serious problem: trash/litter & 0.374 & 0.207 & 0.071 \\
\hline Serious problem: poor sidewalks & -0.078 & 0.178 & 0.661 \\
\hline \multicolumn{4}{|c|}{ Census Tract Level Variables } \\
\hline Census tract: top quintile black/AA* & -0.332 & 0.158 & 0.036 \\
\hline Census tract over $\$ 50,600 \mathrm{MAHI}$ & -0.063 & 0.209 & 0.764 \\
\hline Census tract $>20 \%$ Bachelor deg & -0.147 & 0.193 & 0.447 \\
\hline Census tract $>80 \%$ HS diploma & 0.008 & 0.194 & 0.968 \\
\hline
\end{tabular}


...the fit between the observed errors and the theoretical expectation. Values under 1.00 indicate underdispersion, and values greater than 1.00 indicate overdispersion. Overdispersion and underdispersion may indicate model misspecification, overly influential outliers, or the exclusion of an important level in the model.

\section{Physical Activity in Least Advantaged Census Tracts (Exam 1)}

This analysis was conducted in SAS using Proc Reg (regression) since the neighborhood level variables are controlled by definition (least advantaged census tracts). The results for the total physical activity score in exam 1 for the overall study sample are in Table 5. The model's adjusted $\mathrm{R}^{2}=.1758$ indicating that $17.58 \%$ of the variance in the dependent variable is explained by independent variables in the model.

\section{Analysis of Physical Activity Results}

As expected, the amount of physical activity is impacted by demographic variables such as age, education, and income. It is also expected that higher body mass index and past hospitalizations due to myocardial infarction predict significantly lower physical activity scores. Of particular interest, however, are the $\mathrm{M}^{3}$ Ecosocial Framework variables. In the overall study sample, people with a larger social network size had significantly higher physical activity scores compared with those with a small social network size as hypothesized.

In addition, people who lived in census tracts that were in the top quintile of percentage of African American residents were significantly less likely to have higher physical activity scores. Social network size and percentage of African Americans in a census tract were significant in the overall study sample model. Participants who reported a serious problem with trash/litter in their neighborhood were likely to have higher physical activity scores than people who do not report either of the aforementioned. The direction of the impact of this variable was unexpected however.

When analyzing physical activity predictors in the least advantaged census tracts in comparison with the overall study sample, car ownership becomes significant when compared with results from the overall sample. This most likely means that in the least advantaged census tracts, being able to drive to parks, playgrounds, or exercise facilities is important when those facilities may not be close in proximity.

It may be that people who exercise and walk around their neighborhood may be more likely to notice the serious problem with trash and litter in their neighborhood. Or it may be that some persons may choose vigilance as a response and work to find a way around the serious problem. Thus, those with vigilance have a higher physical activity score, but those who are less likely to be vigilant are also less likely to engage in more physical activity. Participants who are characterized by vigilance take responsibility for engaging in a positive health behavior-physical activity. 
Table 5: Results for Physical Activity Score in Exam 1 in Least Advantaged Census Tracts

\begin{tabular}{lccc}
\hline Variable & B (Slope) & Std. Error & p-Value \\
\hline \multicolumn{1}{c}{ Literature-Based Covariates } & & \\
Age at time of exam 1* & -0.054 & 0.013 & $<.0001$ \\
Male gender & -0.134 & 0.319 & 0.676 \\
College degree & 0.220 & 0.307 & 0.474 \\
Income category & 0.209 & 0.156 & 0.182 \\
Personal wealth $=\$ 200,000+$ & 0.575 & 0.656 & 0.381 \\
Car ownership* & 1.118 & 0.491 & 0.024 \\
Past or current smoker & 0.503 & 0.301 & 0.097 \\
Consumed alcohol in past year* & 0.624 & 0.312 & 0.046 \\
Body mass index* & -0.059 & 0.018 & 0.001 \\
Past heart attack* & -1.370 & 0.572 & 0.017 \\
& $\quad \mathbf{M}^{3}$ Ecosocial Framework Variables & \\
Church attendance $>$ 1x/week & 0.525 & 0.398 & 0.188 \\
Prays frequently $>$ 1x/day & -0.138 & 0.419 & 0.742 \\
Feel God's love for me $>$ 1x/day & -0.218 & 0.360 & 0.546 \\
Passive problem solving score & -0.023 & 0.046 & 0.616 \\
Stress due to neighborhood crime & 0.354 & 0.294 & 0.229 \\
Social network size: $3+$ friends & 0.406 & 0.273 & 0.139 \\
Serious problem: excessive noise & 0.026 & 0.418 & 0.951 \\
Serious problem: heavy traffic & -0.337 & 0.323 & 0.298 \\
Serious problem: lack of parks & -0.266 & 0.400 & 0.507 \\
Serious problem: trash/litter* & 1.036 & 0.399 & 0.010 \\
Serious problem: poor sidewalks & 0.016 & 0.358 & 0.965 \\
\hline
\end{tabular}

* p-value significant at the 0.05 level; $n=338$ in 12 census tracts 


\section{Hypertension Status (Exam 2)}

\section{Hypertension Status in Overall Study Sample (Exam 2)}

This analysis was conducted in SAS using Proc Glimmix to control for Level 1 and Level 2 variables. The results for hypertension status in exam 2 for the overall study sample are in Table 6. The model's generalized chi-square/df $=1.04$, indicating an excellent fit of the independent variables with the values of the dependent variable.

\section{Hypertension Status in Least Advantaged Census Tracts (Exam 2)}

This analysis was conducted in SAS using Proc Logistic since neighborhood (census tract) factors are controlled by definition as least advantaged census tracts. The results for hypertension status in exam 2 for the overall study sample are in Table 7. The model is significant overall and the $\mathrm{c}$ statistic $=0.828$, indicating a good fit of the independent variables with the values of the dependent variable.

\section{Analysis of Hypertension Status Results}

None of the $\mathrm{M}^{3}$ Ecosocial Framework variables are significant in either the overall study sample or among those living in the least advantaged census tracts. However, results indicate that education may play an important role in explaining who is diagnosed with hypertension. In the overall sample analysis, having a college degree confers protection against being hypertensive.

Attending college may expose persons to better information regarding health and nutrition. An alternative explanation is the correlation with social status. People with a college degree are likely to have a higher status in the job force and higher employment rates when compared to those with only a high school diploma. The differences in opportunities and status may be correlating with the amount of stress (which impacts high blood pressure) that is experienced by persons depending on their education. Social status - how persons perceive other people based on their educational background - may explain why education is significant, but not income.

The idea that hypertension is affected by social status is not new. In his introduction to the book Why are Some People Healthy and Othe rs Not? The Determinants of Health of Populations, Robert Evans (1994) summarizes animal and human research showing a distinct link between social status and health outcomes (based on dominance status for animals or rank in work environments for humans). In his book Mind the Gap: Hierarchies, Health and Human Evolution , Richard Wilkinson (2001) indicates that hierarchies in the social order are characterized by higher levels of sustained stress for individuals at the bottom of the social order. Wilkinson (2001, p. 42) also notes that "the accumulated physiological response to chronic stress" is called 'allostatic load.' One of the primary results of chronic stress is elevated blood pressure. Thus, social status-via increased allostatic loads for people with low social status - can help explain why some people have higher risk for being diagnosed with hypertension compared with others. 
Table 6: Hypertension Status in Exam 2 in Overall Study Sample

\begin{tabular}{|c|c|c|c|c|c|c|}
\hline \multirow[t]{2}{*}{$\begin{array}{l}\text { Variable } \\
\end{array}$} & $\begin{array}{c}\text { B } \\
\text { (Slope) }\end{array}$ & \multirow{2}{*}{$\begin{array}{c}\text { Std. } \\
\text { Error }\end{array}$} & \multirow[t]{2}{*}{ p-Value } & \multirow[t]{2}{*}{$\begin{array}{l}\text { Odds } \\
\text { Ratio } \\
\end{array}$} & \multicolumn{2}{|c|}{$\frac{\text { Odds Ratio }}{95 \% \text { CI }}$} \\
\hline & Literature-Based Covariates & & & & & \\
\hline Age at time of exam $1^{*}$ & 0.082 & 0.009 & $<.0001$ & 1.086 & 1.066 & 1.105 \\
\hline Male gender & -0.119 & 0.171 & 0.486 & 0.888 & 0.635 & 1.241 \\
\hline College degree* & -0.480 & 0.230 & 0.037 & 0.619 & 0.394 & 0.971 \\
\hline Income category & 0.056 & 0.100 & 0.576 & 1.058 & 0.869 & 1.287 \\
\hline Personal wealth $=\$ 200,000+$ & 0.015 & 0.498 & 0.976 & 1.015 & 0.382 & 2.699 \\
\hline Car ownership & -0.225 & 0.255 & 0.377 & 0.799 & 0.485 & 1.316 \\
\hline Past or current smoker & 0.138 & 0.187 & 0.462 & 1.148 & 0.795 & 1.657 \\
\hline Total physical activity score & 0.009 & 0.035 & 0.789 & 1.010 & 0.942 & 1.082 \\
\hline Consumed alcohol in past year* & -0.357 & 0.162 & 0.027 & 0.700 & 0.510 & 0.961 \\
\hline Body mass index* & 0.056 & 0.012 & $<.0001$ & 1.058 & 1.034 & 1.082 \\
\hline Health insurance coverage & -0.100 & 0.284 & 0.724 & 0.905 & 0.519 & 1.579 \\
\hline Ever used home remedy & 0.166 & 0.154 & 0.280 & 1.181 & 0.874 & 1.596 \\
\hline Past heart attack & 0.776 & 0.582 & 0.183 & 2.172 & 0.693 & 6.805 \\
\hline \multicolumn{7}{|c|}{$M^{3}$ Ecosocial Framework Variables } \\
\hline Church attendance $>1 \mathrm{x} /$ week & -0.329 & 0.216 & 0.127 & 0.720 & 0.471 & 1.098 \\
\hline Prays frequently $>1 \mathrm{x} /$ day & -0.094 & 0.217 & 0.664 & 0.910 & 0.595 & 1.392 \\
\hline Feel God's love for me $>1 \mathrm{x} /$ day & 0.213 & 0.196 & 0.279 & 1.237 & 0.842 & 1.818 \\
\hline Passive problem solving score & 0.018 & 0.029 & 0.531 & 1.018 & 0.962 & 1.078 \\
\hline Trust in medical provider & -0.165 & 0.523 & 0.753 & 0.848 & 0.304 & 2.366 \\
\hline Satisfaction with health care & 0.631 & 0.609 & 0.301 & 1.879 & 0.568 & 6.212 \\
\hline Unfair medical treatment & 0.043 & 0.230 & 0.851 & 1.044 & 0.665 & 1.639 \\
\hline Difficulty reaching health services & 0.346 & 0.334 & 0.302 & 1.413 & 0.733 & 2.723 \\
\hline Social network size: $3+$ friends & -0.087 & 0.155 & 0.575 & 0.917 & 0.677 & 1.242 \\
\hline Stress due to neighborhood crime & 0.192 & 0.198 & 0.333 & 1.212 & 0.821 & 1.788 \\
\hline Low medical transport support & -0.164 & 0.367 & 0.654 & 0.848 & 0.413 & 1.743 \\
\hline Serious problem: excessive noise & 0.036 & 0.283 & 0.899 & 1.037 & 0.595 & 1.805 \\
\hline Serious problem: heavy traffic & 0.003 & 0.216 & 0.989 & 1.003 & 0.656 & 1.533 \\
\hline Serious problem: grocery access & -0.583 & 0.331 & 0.079 & 0.558 & 0.292 & 1.069 \\
\hline Serious problem: lack of parks & 0.097 & 0.242 & 0.688 & 1.102 & 0.685 & 1.772 \\
\hline Serious problem: trash/litter & -0.109 & 0.258 & 0.673 & 0.897 & 0.540 & 1.488 \\
\hline Serious problem: poor sidewalks & -0.283 & 0.226 & 0.211 & 0.754 & 0.484 & 1.174 \\
\hline \multicolumn{7}{|c|}{ Census Tract Level Variables } \\
\hline Census tract: top quintile black/AA & 0.210 & 0.204 & 0.303 & 1.233 & \multicolumn{2}{|c|}{ Not generated } \\
\hline Census tract over $\$ 50,600$ MAHI & -0.337 & 0.255 & 0.187 & 0.714 & \multicolumn{2}{|c|}{ Not generated } \\
\hline Census tract $>80 \%$ HS diploma & 0.361 & 0.237 & 0.129 & 1.434 & \multicolumn{2}{|c|}{ Not generated } \\
\hline Census tract $>20 \%$ Bachelor deg & 0.138 & 0.246 & 0.576 & 1.148 & \multicolumn{2}{|c|}{ Not generated } \\
\hline FF restaurants in top quintile & -0.132 & 0.137 & -0.337 & 0.877 & \multicolumn{2}{|c|}{ Not generated } \\
\hline
\end{tabular}


Table 7: Hypertension Status in Exam 2 in Least Advantaged Census Tracts

\begin{tabular}{|c|c|c|c|c|c|c|}
\hline Variable & $\begin{array}{c}\text { B } \\
\text { (slope) }\end{array}$ & $\begin{array}{l}\text { Std. } \\
\text { Error }\end{array}$ & p-Value & $\begin{array}{l}\text { Odds } \\
\text { ratio }\end{array}$ & $\frac{\text { Odds }}{95 \%}$ & $\frac{\text { Ratio }}{\text { CI }}$ \\
\hline Litera & ature-B & ased $\mathrm{Cr}$ & ovariates & & & \\
\hline Age at time of exam $1 *$ & 0.057 & 0.020 & 0.005 & 1.059 & 1.017 & 1.102 \\
\hline Male gender & 0.826 & 0.513 & 0.108 & 2.283 & 0.835 & 6.241 \\
\hline College degree & -0.354 & 0.489 & 0.469 & 0.702 & 0.269 & 1.829 \\
\hline Income category & 0.033 & 0.248 & 0.893 & 1.034 & 0.636 & 1.679 \\
\hline Personal wealth $=\$ 200,000+$ & 1.039 & 0.942 & 0.270 & 2.826 & 0.446 & 17.90 \\
\hline Car ownership & -0.801 & 0.851 & 0.347 & 0.449 & 0.085 & 2.379 \\
\hline Past or current smoker & 0.705 & 0.472 & 0.136 & 2.023 & 0.802 & 5.104 \\
\hline Total physical activity score & -0.007 & 0.095 & 0.945 & 0.993 & 0.825 & 1.197 \\
\hline Consumed alcohol in past year* & -1.124 & 0.463 & 0.015 & 0.325 & 0.131 & 0.805 \\
\hline Body mass index* & 0.078 & 0.033 & 0.017 & 1.081 & 1.014 & 1.152 \\
\hline Health insurance coverage* & 1.429 & 0.564 & 0.011 & 4.175 & 1.383 & 12.61 \\
\hline Ever used home remedy & -0.002 & 0.429 & 0.996 & 0.998 & 0.430 & 2.315 \\
\hline Past heart attack & 2.011 & 1.824 & 0.270 & 7.472 & 0.209 & 266.52 \\
\hline$M^{3} E \operatorname{coso}$ & ocial Fr: & amewor & rk Variab & & & \\
\hline Church attendance $>1 \mathrm{x} /$ week & -0.741 & 0.622 & 0.234 & 0.477 & 0.141 & 1.614 \\
\hline Prays frequently $>1 \mathrm{x} /$ day & 0.245 & 0.677 & 0.717 & 1.278 & 0.339 & 4.816 \\
\hline Feel God's love for me $>1 \mathrm{x} /$ day & -0.478 & 0.602 & 0.427 & 0.620 & 0.191 & 2.017 \\
\hline Passive problem solving score & 0.144 & 0.082 & 0.078 & 1.155 & 0.984 & 1.356 \\
\hline Trust in medical provider & 0.016 & 1.705 & 0.993 & 1.016 & 0.036 & 28.709 \\
\hline Satisfaction with health care & 2.453 & 2.031 & 0.227 & 11.626 & 0.217 & 623.15 \\
\hline Unfair medical treatment & -0.435 & 0.583 & 0.456 & 0.648 & 0.207 & 2.028 \\
\hline Difficulty reaching health services & 0.087 & 0.845 & 0.918 & 1.091 & 0.208 & 5.715 \\
\hline Social network size: $3+$ friends & -0.143 & 0.448 & 0.750 & 0.867 & 0.361 & 2.085 \\
\hline Stress due to neighborhood crime & 0.243 & 0.471 & 0.606 & 1.276 & 0.506 & 3.213 \\
\hline Low medical transport support & 0.156 & 0.907 & 0.863 & 1.169 & 0.197 & 6.919 \\
\hline Serious problem: excessive noise & -1.179 & 0.647 & 0.069 & 0.307 & 0.086 & 1.093 \\
\hline Serious problem: heavy traffic & 0.243 & 0.492 & 0.621 & 1.275 & 0.486 & 3.345 \\
\hline Serious problem: grocery access & -0.196 & 0.758 & 0.796 & 0.822 & 0.186 & 3.630 \\
\hline Serious problem: lack of parks & 0.709 & 0.714 & 0.321 & 2.031 & 0.501 & 8.226 \\
\hline Serious problem: trash/litter & 0.329 & 0.599 & 0.583 & 1.390 & 0.430 & 4.498 \\
\hline Serious problem: poor sidewalks & -0.054 & 0.548 & 0.922 & 0.948 & 0.324 & 2.772 \\
\hline
\end{tabular}

* p-value significant at the 0.05 level; $\mathrm{n}=222$ in 15 census tracts 
When comparing the two statistical models predicting hypertension status, having a college degree is significant in the overall study sample, but not among those living in the least advantaged census tracts. This provides an indication that social status, more than exposure to better information regarding health and nutrition, might explain who is diagnosed with hypertension in Exam 2 and who is not. Presumably, the exposure to better information would be the same for persons with a college degree regardless of neighborhood of residence. However, it is easy to see how social status may clearly correspond with a person's neighborhood of residence. In social settings, people often ask, "What part of town do you live in?" This might influence how persons are subsequently treated - better if they live in a more advantaged neighborhood, worse if they live in a less advantaged one.

But even more so, living in a least advantaged census tract may be deleterious to one's blood pressure regardless of college degree attainment. This may be due to factors such as heightened police surveillance, redlining, or predatory financial lending practices that are more likely to take place in least advantaged neighborhoods. However, these types of factors were not measured in the study.

Whatever the case, a more nuanced argument for social status emerges. Hypertension, as influenced by social status, may not only be attributed to the level of education one might possess, but where one lives. In other words, social status, among participants of the Jackson Heart Study, may be both a function of having a college degree and living in a particular neighborhood. To the degree that both having a college degree and living in a certain neighborhood will determine the status of an individual in a social setting, social status would then be likely to determine the frequency and intensity of stressful interactions a person may experience. This would mean that African Americans who live in a more advantaged neighborhood and have a college degree are less likely to be exposed to stress-inducing interactions compared to those who live in less advantaged neighborhoods (whether they have a college degree or not). Therefore, the role of neighborhoods is implicated although none of the neighborhood variables proposed in the $\mathrm{M}^{3}$ Ecosocial Framework was significant.

Other variables were also marginally significant. In the overall sample, people who had a serious problem with grocery access had a reduced risk of having hypertension in Exam $2(p$-value $=0.079)$ compared to those who did not report having a serious problem with grocery access. Among people living in the least advantaged census tracts, persons who reported having a serious problem with excessive noise were at less risk for having hypertension in Exam 2 ( -value $=0.069$ ) compared to those who did not have a serious problem with excessive noise. These results, as with the physical activity statistical results, are also likely due to persons being vigilant in the face of serious neighborhood problems.

Also, among those living in the least advantaged census tracts, persons with higher passive problem solving scores (i.e. being more passive in solving problems) were found to have increased odds for hypertension status in Exam 2 ( $p$-value $=0.078$ ) compared to those with lower passive problem solving scores. The role of passive problem solving is 
implicated in predicting an undesired health outcome. The variable acts in the manner hypothesized. People with higher passive problem solving scores have increased odds for being diagnosed with hypertension in Exam 2.

\section{Diabetes Status (Exam 2)}

\section{Diabetes Status in Overall Study Sample (Exam 2)}

This analysis was conducted in SAS using Proc Glimmix. The results for diabetes status in exam 2 for the overall study sample are in Table 8. The model's generalized chi-square/df $=1.01$, indicating an excellent fit of the independent variables with the values of the dependent variable.

\section{Diabetes Status in Least Advantaged Census Tracts (Exam 2)}

This analysis was conducted in SAS using Proc Logistic. The results for diabetes status in exam 2 for the overall study sample are in Table 9. The model is significant overall and the $\mathrm{c}$ statistic $=0.793$, indicating a good fit of the independent variables with the values of the dependent variable.

\section{Analysis of Diabetes Status}

As expected, factors such as age, gender, and body mass index are significant variables in predicting a person being diagnosed with diabetes in Exam 2 in the overall study sample. Behaviors such as alcohol consumption, smoking, and physical activity also play a role in predicting a diagnosis of diabetes. Unlike hypertension however, several $\mathrm{M}^{3}$ Ecosocial Framework factors were significant.

In the overall study sample, diabetes status is predicted by unfair medical treatment. As expected, people who reported experiencing unfair medical treatment were significantly more likely to be diagnosed with diabetes in when compared with those who did not report experiencing unfair medical treatment. In addition, people who live in census tracts with 220 or more fast food restaurants had an increased chance of being diabetic compared with those living in census tracts with less than 220 fast food restaurants.

In the least advantaged census tracts, several $\mathrm{M}^{3}$ Ecosocial Framework factors were all significant predictors of being diabetic in Exam 2. People who reported higher church attendance, high stress due to neighborhood crime, and serious problems with a lack of parks were each significantly less likely to be diabetic. The direction of each of these three results was unexpected. Church attendance confers some protection against diabetes status although the mechanism here is unclear. Some research indicates a link between stress and diabetes via the interruption of glucose storage (Lloyd et al., 2005). Attending church frequently may help decrease levels of stress among African Americans and thereby reduce the risk of a diabetes diagnosis. 
Table 8: Diabetes Status in Exam 2 in Overall Study Sample

\begin{tabular}{|c|c|c|c|c|c|c|}
\hline Variable & $\begin{array}{c}\text { B } \\
\text { (Slope) }\end{array}$ & $\begin{array}{c}\text { Std. } \\
\text { Error }\end{array}$ & p-Value & \multirow[t]{2}{*}{$\begin{array}{l}\text { Odds } \\
\text { Ratio } \\
\end{array}$} & \multicolumn{2}{|c|}{$\frac{\text { Odds Ratio }}{95 \% \mathrm{CI}}$} \\
\hline \multicolumn{6}{|c|}{ Literature-Based Covariates } & \\
\hline Age at time of exam $1^{*}$ & 0.040 & 0.009 & $<.0001$ & 1.041 & 1.023 & 1.060 \\
\hline Male gender* & 0.530 & 0.187 & 0.005 & 1.699 & 1.177 & 2.453 \\
\hline ollege degree & -0.025 & 0.219 & & .975 & 0.634 & 1.500 \\
\hline Income category & -0.124 & 0.102 & & 0.884 & 0.724 & 1.079 \\
\hline Personal wealth $=\$ 200,000+$ & 0.316 & 0.457 & & 1.371 & 0.559 & 3.365 \\
\hline ar ownership & -0.039 & 0.288 & & & 0.547 & 1.690 \\
\hline nt smoker & 0.320 & 0.188 & & 377 & 0.952 & 1.990 \\
\hline Total physical activity score* & -0.073 & 0.036 & & .929 & 0.866 & 0.998 \\
\hline Consumed alcohol in past year* & -0.614 & 0.180 & & 0.541 & 0.380 & 0.770 \\
\hline & 0.096 & 0.013 & & & 1.074 & 1.129 \\
\hline & -0.110 & 0.308 & & & 0.490 & 1.639 \\
\hline & -0.026 & 0.165 & & & 0.704 & 1.347 \\
\hline & 0.484 & 0.436 & 0.267 & 1.623 & 0.690 & 3.819 \\
\hline \multicolumn{7}{|c|}{$\mathbf{M}^{3}$ Ecosocial Framework Variables } \\
\hline hurch attendance $>1 \mathrm{x} /$ week & -0.243 & 0.230 & & & 0.499 & 1.232 \\
\hline & 0.230 & 0.24 & & & 0.776 & 2.041 \\
\hline eel God's love for me $>1 \mathrm{x} /$ day & -0.198 & 0.216 & & 0.821 & 0.537 & 1.253 \\
\hline Passive problem solving score & -0.059 & 0.031 & & 0.942 & 0.887 & 1.001 \\
\hline & & 0.5 & & & 0.669 & 6.258 \\
\hline & -0.319 & 0.631 & & & 0.211 & 2.507 \\
\hline Unfair medical treatment* & 0.493 & 0.228 & & 637 & 1.046 & 2.561 \\
\hline reaching health services & 0.216 & 0.338 & & 1.241 & 0.640 & 2.408 \\
\hline & -0.124 & 0.166 & & & 0.638 & 1.223 \\
\hline & -0.356 & 0.2 & & & & 1.071 \\
\hline & -0.307 & 0.370 & & & 0.356 & 1.522 \\
\hline Serious problem: excessive noise & 0.226 & 0.288 & & 54 & 0.712 & 2.206 \\
\hline Serious problem: heavy traffic & 0.101 & 0.225 & & & 0.712 & 1.720 \\
\hline & 0.195 & & & & 0.5 & 2.470 \\
\hline & -0.529 & 0.292 & & 0.589 & 0.332 & 1.045 \\
\hline & -0.435 & 0.287 & & & 0.369 & 1.137 \\
\hline Serious problem: poor sidewalks & -0.107 & 0.241 & 0.658 & 0.899 & 0.560 & 1.443 \\
\hline \multicolumn{7}{|c|}{ Census Tract Level Variables } \\
\hline & 0.192 & & & & & \\
\hline & 0.201 & & & & & \\
\hline Census tract $>80 \%$ HS diploma & 0.057 & 0.249 & & 1.058 & Not ge & erated \\
\hline Census tract $>20 \%$ Bachelor deg & -0.187 & 0.244 & 0.44 & 0.830 & Not $\mathrm{g}$ & \\
\hline FF restaurants in top quintile* & 0.268 & 0.114 & 0.019 & 1.307 & Not gen & erated \\
\hline
\end{tabular}

* $\mathrm{p}$-value significant at the 0.05 level; $\mathrm{n}=1032$ in 93 census tracts 
Table 9: Diabetes Status in Exam 2 in Least Advantaged Census Tracts

\begin{tabular}{|c|c|c|c|c|c|c|}
\hline Variable & $\begin{array}{c}\text { B } \\
\text { (Slope) }\end{array}$ & $\begin{array}{l}\text { Std. } \\
\text { Error }\end{array}$ & $\begin{array}{c}\text { p- } \\
\text { Value }\end{array}$ & \multirow[t]{2}{*}{$\begin{array}{l}\text { Odds } \\
\text { Ratio } \\
\end{array}$} & \multicolumn{2}{|c|}{$\frac{\text { Odds Ratio }}{95 \% \mathrm{CI}}$} \\
\hline \multicolumn{6}{|c|}{ Literature-Based Covariates } & \\
\hline Age at time of exam 1 & 0.028 & 0.021 & 0.171 & 1.029 & & 1.072 \\
\hline Male gender & 0.398 & 0.446 & & & & \\
\hline College degree & 0.150 & 0.452 & 0.739 & 1.162 & & 2.818 \\
\hline Income category & 0.247 & 0.219 & & & & \\
\hline Personal wealth $=\$ 200,000+$ & 0.566 & 0.967 & 0.559 & 1.761 & & 11.72 \\
\hline Car ownership & -0.583 & 0.915 & 0.524 & 0.558 & & 3.351 \\
\hline & 0.364 & 0.425 & & & & \\
\hline y score & -0.081 & 0.087 & 0.352 & 0.922 & & 1.093 \\
\hline t year* & -1.531 & 0.469 & & & & 0.542 \\
\hline Bod & 0.062 & 0.028 & 0.026 & 1.064 & & 1.124 \\
\hline & 0.569 & 0.714 & 0.426 & & & 7.158 \\
\hline & 0.489 & 0.396 & 0.216 & & 51 & 3.541 \\
\hline & 0.011 & 1.060 & 0.992 & 1.011 & .127 & 8.067 \\
\hline \multicolumn{7}{|c|}{$\mathbf{M}^{3}$ Ecosocial Framework Variables } \\
\hline Church attendance $>1 \mathrm{x} /$ week $^{*}$ & -1.131 & 0.568 & & 0.323 & & 0.981 \\
\hline & 0.773 & 0.668 & & & & 8.019 \\
\hline$>1 x /$ day & -0.726 & 0.549 & 0.186 & & .165 & 1.418 \\
\hline ing score & 0.047 & 0.072 & 0.516 & & 11 & 1.205 \\
\hline & 0.301 & 1.302 & & & & 17.34 \\
\hline & & & & & & \\
\hline & 0.837 & 0.573 & & & 0.751 & 7.102 \\
\hline & & & & & & 24.56 \\
\hline rk size: $3+$ friends & -0.185 & 0.400 & 0.644 & 0.831 & 0.379 & 1.821 \\
\hline de to neignbornood crime & -0.925 & 0.439 & & & & 0.937 \\
\hline Low medical transport support & -0.174 & 0.694 & 0.803 & 0.841 & 0.216 & 3.274 \\
\hline s problem: excessive noise & 0.124 & 0.582 & & & & 3.545 \\
\hline Serious problem: heavy traffic & 0.373 & 0.482 & 0.439 & 1.452 & 0.564 & 3.738 \\
\hline & 0.375 & 0.772 & 0.627 & 1.455 & 0.321 & 6.603 \\
\hline Serious problem: lack of parks* & & 0.662 & & & 0.073 & 0.979 \\
\hline Serious problem: trash/litter & -0.159 & 0.570 & 0.780 & 0.853 & 0.279 & 2.607 \\
\hline Serious problem: poor sidewalks & 0.571 & 0.536 & 0.286 & 1.770 & 0.619 & 5.059 \\
\hline
\end{tabular}

p-value significant at the 0.05 level; $n=217$ in 15 census tracts 
It is also possible that another mechanism related to church attendance might be the higher amounts of physical activity among people who attend church compared to those who do not. Church attendance is significant for persons living in least advantaged census tracts, but not in the overall sample. Thus, for people living in the least advantaged census tracts, attending church more frequently may be resulting in more physical activity than those who do not attend church frequently. With a lack of spaces for physical activity, the church may constitute one of the few places where persons can increase the amount of their physical activity. The African American church is often characterized by its participatory nature which includes expressive modes of worshipclapping hands, swaying to the music, dancing - when compared to European American churches (Lincoln \& Mamiya, 2003). These worship activities might constitute the methods through which physical activity is working to reduce stress and perhaps function as a form of exercise. Whatever the mechanism, other researchers have discussed the link between church attendance and improved health outcomes, including life expectancy (McCullough et al., 2000; Hall 2006a; Schnall et al., 2010). However, explanations for this phenomenon are still in dispute and not fully understood.

In accordance with the vigilance proposition discussed earlier (in the physical activity analysis section), people reporting high stress due to neighborhood crime and serious problems with a lack of parks and playgrounds may be persons who are aware of certain issues in their neighborhoods and then proceed to develop ways of working around the issues. This would highlight the role of vigilance as a component of high health stewardship in protecting against diabetes.

\section{Hypercholesterolemia Status (Exam 1)}

\section{Hypercholesterolemia Status in Overall Study Sample (Exam 1)}

This analysis was conducted in SAS. The results for hypercholesterolemia status in exam 1 for the overall study sample are in Table 10. The model's generalized chisquare/df $=1.04$, indicating a good fit of the independent variables with the values of the dependent variable.

\section{Hypercholesterolemia Status in Least Advantaged Census Tracts (Exam 1)}

This analysis was conducted in SAS using Proc Logistic. The results for hypercholesterolemia status in exam 1 for the overall study sample are in Table 11. The model is near significant overall and the $\mathrm{c}$ statistic $=0.740$, indicating a good fit of the independent variables with the values of the dependent variable.

\section{Analysis of Hypercholesterolemia Status Results}

Results of this cross sectional analysis indicate that a past heart attack, diabetes status (in the overall study sample), and hypertension status (in the least advantaged census tracts) were the strongest factors in predicting persons who would be classified as having 
Table 10: Hypercholesterolemia Status in Exam 1 in Overall Study Sample

\begin{tabular}{|c|c|c|c|c|c|c|}
\hline Variable & $\begin{array}{c}\text { B } \\
\text { (Slope) }\end{array}$ & $\begin{array}{c}\text { Std. } \\
\text { Error }\end{array}$ & $\begin{array}{c}\text { p- } \\
\text { Value }\end{array}$ & $\begin{array}{l}\text { Odds } \\
\text { Ratio }\end{array}$ & \multicolumn{2}{|c|}{$\frac{\text { Odds Ratio }}{95 \% \mathrm{CI}}$} \\
\hline \multicolumn{7}{|c|}{ Literature-Based Covariates } \\
\hline Age at time of exam $1^{*}$ & 0.022 & 0.008 & 0.004 & 1.022 & 1.007 & 1.037 \\
\hline Male gender & 0.188 & 0.155 & 0.225 & 1.207 & 0.891 & 1.635 \\
\hline College degree & 0.117 & 0.181 & 0.519 & 1.124 & 0.787 & 1.605 \\
\hline Income category & -0.062 & 0.085 & 0.466 & 0.940 & 0.796 & 1.110 \\
\hline Personal wealth $=\$ 200,000+$ & 0.494 & 0.385 & 0.200 & 1.638 & 0.770 & 3.484 \\
\hline Car ownership & 0.096 & 0.234 & 0.682 & 1.101 & 0.695 & 1.743 \\
\hline Past or current smoker & 0.115 & 0.157 & 0.462 & 1.122 & 0.825 & 1.526 \\
\hline Total physical activity score & -0.058 & 0.030 & 0.057 & 0.944 & 0.890 & 1.002 \\
\hline Consumed alcohol in past year & -0.163 & 0.148 & 0.270 & 0.849 & 0.636 & 1.135 \\
\hline Body mass index & -0.009 & 0.010 & 0.379 & 0.991 & 0.971 & 1.011 \\
\hline Health insurance coverage & 0.093 & 0.251 & 0.712 & 1.097 & 0.671 & 1.795 \\
\hline Ever used home remedy & -0.209 & 0.136 & 0.126 & 0.812 & 0.621 & 1.061 \\
\hline Past heart attack* & 1.343 & 0.386 & 0.001 & 3.830 & 1.794 & 8.175 \\
\hline Diabe & 0.714 & 0.184 & 0.000 & 2.042 & 1.423 & 2.931 \\
\hline Hypertension status & 0.250 & 0.157 & 0.113 & 1.284 & 0.943 & 1.748 \\
\hline \multicolumn{7}{|c|}{$M^{3}$ Ecosocial Framework Variables } \\
\hline Church attendance $>1 \mathrm{x} /$ week & -0.161 & 0.198 & 0.419 & 0.852 & 0.577 & 1.257 \\
\hline Prays frequently $>1 \mathrm{x} /$ day & 0.087 & 0.204 & 0.6 & 1.091 & 0.731 & 1.628 \\
\hline s love for $m e>1 x /$ day & 0.153 & 0.183 & 0.402 & 1.165 & 0.814 & 1.668 \\
\hline Passive problem solving score & 0.004 & 0.025 & 0.885 & 1.004 & 0.956 & 1.054 \\
\hline Trust in medical provider & 0.013 & 0.465 & 0.978 & 1.013 & 0.407 & 2.522 \\
\hline Satisfaction with health care & 0.667 & 0.621 & 0.283 & 1.949 & 0.576 & 6.596 \\
\hline Unfair medical treatment & -0.154 & 0.208 & 0.461 & 0.858 & 0.570 & 1.290 \\
\hline Difficulty reaching health services & -0.085 & 0.280 & 0.762 & 0.919 & 0.530 & 1.592 \\
\hline Social network size: $3+$ friends & -0.072 & 0.137 & 0.600 & 0.931 & 0.712 & 1.217 \\
\hline Stress due to neighborhood crime & 0.262 & 0.172 & 0.129 & 1.299 & 0.927 & 1.820 \\
\hline Low medical transport support & 0.370 & 0.297 & 0.212 & 1.448 & 0.809 & 2.593 \\
\hline Serious problem: excessive noise & -0.253 & 0.248 & 0.307 & 0.776 & 0.477 & 1.262 \\
\hline Serious problem: heavy traffic & -0.011 & 0.189 & 0.953 & 0.989 & 0.683 & 1.432 \\
\hline Serious problem: grocery access & -0.378 & 0.315 & 0.230 & 0.685 & 0.369 & 1.271 \\
\hline Serious problem: lack of parks & 0.087 & 0.231 & 0.706 & 1.091 & 0.693 & 1.716 \\
\hline Serious problem: trash/litter & -0.093 & 0.236 & 0.693 & 0.911 & 0.573 & 1.448 \\
\hline Serious problem: poor sidewalks & 0.012 & 0.203 & 0.954 & 1.012 & 0.679 & 1.507 \\
\hline \multicolumn{7}{|c|}{ Census Tract Level Variables } \\
\hline Census tract $>93.3 \%$ black/AA & -0.086 & 0.164 & 0.596 & 0.917 & \multicolumn{2}{|c|}{ Not generated } \\
\hline Census tract over $\$ 50,600 \mathrm{MAHI}$ & -0.100 & 0.220 & 0.653 & 0.905 & \multicolumn{2}{|c|}{ Not generated } \\
\hline Census tract $>80 \%$ HS diploma & -0.080 & 0.204 & 0.697 & 0.923 & \multicolumn{2}{|c|}{ Not generated } \\
\hline Census tract $>20 \%$ Bachelor deg & 0.307 & 0.198 & 0.121 & 1.360 & \multicolumn{2}{|c|}{ Not generated } \\
\hline FF restaurants in top quintile & 0.102 & 0.097 & 0.294 & 1.108 & Not get & nerated \\
\hline
\end{tabular}

* p-value significant at the 0.05 level; $\mathrm{n}=1227$ in 93 census tracts 
Table 11: Hypercholesterolemia Status in Exam 1 in Least Advantaged Census Tracts

\begin{tabular}{|c|c|c|c|c|c|c|}
\hline \multicolumn{2}{|c|}{$\begin{array}{cc}\text { Variable } & \text { B } \\
& \text { (Slope) } \\
\end{array}$} & $\begin{array}{l}\text { Std. } \\
\text { Error }\end{array}$ & \multirow{2}{*}{$\begin{array}{c}\text { p- } \\
\text { Value } \\
\text { iates }\end{array}$} & \multirow[t]{2}{*}{$\begin{array}{l}\text { Odds } \\
\text { Ratio } \\
\end{array}$} & \multicolumn{2}{|c|}{$\frac{\text { Odds Ratio }}{95 \% \text { CI }}$} \\
\hline \multicolumn{5}{|c|}{ Literature-Based Covariates } & & \\
\hline ge at time of exam 1 & -0.010 & 0.017 & 0.550 & & & 1.024 \\
\hline & & & & & & \\
\hline & 0.072 & 0.370 & & 1.075 & & 2.221 \\
\hline & & & & & & 1.522 \\
\hline al wealth $=\$ 200,000+$ & 1.253 & & & & & 18.668 \\
\hline ship & -0.889 & 89 & & 0.411 & & 2.346 \\
\hline & & & & & & 2.078 \\
\hline & -0.096 & .074 & & 0.909 & & 1.050 \\
\hline & & & & & & 2.157 \\
\hline & 0.038 & 24 & & 62 & & 1.009 \\
\hline & 0.243 & & & & & 2.091 \\
\hline & 0.398 & 0.327 & & & & 1.276 \\
\hline & & & & & & 47.402 \\
\hline & & & & & & 3.525 \\
\hline & 0.803 & 0.405 & 0.047 & & & 4.941 \\
\hline \multicolumn{7}{|c|}{$\mathbf{M}^{3}$ Ecosocial Framework Variables } \\
\hline & 0.597 & & & & & 5.196 \\
\hline & & & & & & 2.333 \\
\hline $1 x /$ day & & & & & & 3.853 \\
\hline & & & & & & \\
\hline & -1.060 & & & & & 3.736 \\
\hline & & & & & & 212.33 \\
\hline & & & & & & 2.098 \\
\hline & & & & & & 3.228 \\
\hline & 0.044 & & & & & 2.017 \\
\hline & & & & & & \\
\hline sport support & 0.056 & 0.665 & .932 & 1.058 & 0.287 & 3.895 \\
\hline & -0.405 & 0.551 & & & & 1.963 \\
\hline & & & & & & 2.070 \\
\hline & -1.728 & 0.932 & & & & 1.103 \\
\hline parks & & & & & & 4.884 \\
\hline & -0.439 & & & & & 1.680 \\
\hline Serious problem: poor sidewalks & -0.751 & 0.454 & 0.098 & 0.472 & 0.194 & 1.148 \\
\hline
\end{tabular}

* p-value significant at the 0.05 level; $\mathrm{n}=241$ in 15 census tracts 
high cholesterol in the overall sample in Exam 1. Physical activity score was near significant $(p$-value $=0.057)$ indicating a likely impact on high cholesterol.

No $\mathrm{M}^{3}$ Ecosocial Framework variable was significant. However, two measures of problems in the neighborhood were near significant in the least advantaged census tract model: grocery access ( $p$-value $=0.064)$ and poor sidewalks $(p$-value $=0.098)$. Measures of serious problems in the neighborhood are significant (in the physical activity and diabetes statistical models) or near significant (in the hypercholesterolemia statistical model) only among people living in the least advantaged census tracts. Another way of stating these finding is this: a lack of vigilance, when living in a least advantaged census tract, can lead to poorer health behaviors (physical activity) and poor health outcomes (diabetes and hypercholesterolemia) comparing to being vigilant. Vigilance serves as a protective factor against a lack of health-enhancing facilities in the least advantaged neighborhoods.

\section{Hospitalization due to Ambulatory Care Sensitive Condition (ACSC)}

This analysis was conducted in SAS using Proc Glimmix. The model is the same as the one for diabetes and hypertension status with two changes: the "satisfaction with health care" variable and the "religion helps greatly with stress" variable were not included. These changes were made when the model would not converge with the standard model. The results for hospitalizations due to ambulatory care sensitive conditions for the overall study sample are in Table 12. The results cover a time frame from 2000 to 2009 . The model's generalized chi-square $/ \mathrm{df}=0.85$, indicating a degree of underdispersion.

Results for this model indicate that only one variable was significant. People with higher ages had increased odds of being hospitalized due to an ACSC. Persons who were diagnosed with hypertension were also nearly 3 times more likely to experience an ACSC hospitalization. However, this variable was only near significant ( $\mathrm{p}$-value $=0.084)$. The lack of statistically significant variables is likely due the limited variability in the dependent variable. The relatively small percentage of ACSC hospitalization prevented an analysis of ACSC hospitalizations in the least advantaged census tracts. Out of the 1498 persons living in the least advantaged census tracts, only 22 experienced an ACSC hospitalization.

\section{Summary Analysis}

Here are the hypotheses that were posed earlier in the chapter followed by findings (decision rule) based on the statistical analyses in Table 13.

Overall, the findings are mixed. Some variables used to test the $\mathrm{M}^{3}$ Ecosocial Framework are statistically significant while others are not. Based on study results, there is no evidence to support hypotheses B, C, and E. None of the factors in those hypotheses was significant in any of the statistical models. Evidence is found to support 
Table 12: $\quad$ ACSC Hospitalization from 2000-2009

\begin{tabular}{|c|c|c|c|c|c|c|}
\hline \multirow[t]{2}{*}{ Variable } & $\begin{array}{c}\text { B } \\
\text { (Slope) }\end{array}$ & $\begin{array}{c}\text { Std. } \\
\text { Error }\end{array}$ & p-Value & \multirow[t]{2}{*}{$\begin{array}{l}\text { Odds } \\
\text { Ratio }\end{array}$} & \multicolumn{2}{|c|}{$\frac{\text { Odds Ratio }}{95 \% \mathrm{CI}}$} \\
\hline & \multicolumn{5}{|c|}{ Literature-Based Covariates } & \\
\hline Age at time of exam $1 *$ & 0.068 & 0.022 & 0.002 & 1.070 & 1.024 & 1.118 \\
\hline Male gender & 0.297 & 0.465 & 0.523 & 1.346 & 0.541 & 3.349 \\
\hline College degree & -0.536 & 0.540 & 0.321 & 0.585 & 0.203 & 1.687 \\
\hline Income category & -0.106 & 0.260 & 0.684 & 0.900 & 0.540 & 1.498 \\
\hline Personal wealth $=\$ 200,000+$ & 0.697 & 1.155 & 0.546 & 2.008 & 0.208 & 19.35 \\
\hline Car ownership & -1.044 & 0.825 & 0.206 & 0.352 & 0.070 & 1.776 \\
\hline Past or current smoker & -0.574 & 0.476 & 0.228 & 0.563 & 0.222 & 1.433 \\
\hline Total physical activity score & 0.129 & 0.097 & & 1.138 & 0.941 & 1.375 \\
\hline Consumed alcohol in past year & 0.640 & 0.465 & 0.169 & 1.896 & 0.762 & 4.719 \\
\hline Body $n$ & -0.002 & 0.036 & & 0.998 & 0.930 & 1.071 \\
\hline e coverage & 0.016 & 0.859 & 0.985 & 1.016 & 0.188 & 5.478 \\
\hline Ever $\mathrm{l}$ & 0.043 & 0.415 & & 1.044 & 0.463 & 2.356 \\
\hline Past heart attack & -0.947 & 1.156 & 0.4 & 0.388 & 0.040 & 3.746 \\
\hline & 0.094 & 0.519 & & 1.099 & 0.397 & 3.045 \\
\hline & 1.038 & 0.600 & & & 0.870 & 9.168 \\
\hline Hypercholesterolemia status & 0.566 & 0.415 & 0.173 & 1.761 & 0.780 & \\
\hline \multicolumn{7}{|c|}{$\mathbf{M}^{3}$ Ecosocial Framework Variables } \\
\hline Church attendance $>1 \mathrm{x} /$ week & -0.573 & 0.562 & & 0.564 & 0.187 & 1.699 \\
\hline & -0.509 & 0.577 & & & 0.194 & 1.864 \\
\hline e for $m e>1 x /$ day & -0.078 & 0.549 & 0.887 & 0.925 & 0.315 & 2.716 \\
\hline Religion helps greatly with stress & 0.470 & 0.525 & 0.371 & 1.599 & 0.571 & 4.480 \\
\hline Passive problem solving score & 0.031 & 0.074 & 0.680 & 1.031 & 0.891 & 1.193 \\
\hline Trust in medical provider & -0.353 & 1.139 & & 0.702 & 0.075 & 6.566 \\
\hline lical treatment & 0.592 & 0.536 & & 1.808 & 0.632 & 5.173 \\
\hline aching health services & -0.270 & 0.860 & 0.754 & 0.763 & 0.141 & 4.129 \\
\hline Social network size: $3+$ friends & 0.699 & 0.457 & 0.126 & 2.012 & 0.821 & 4.933 \\
\hline Stress due to neighborhood crime & -0.369 & 0.429 & 0.389 & 0.691 & 0.298 & 1.602 \\
\hline Low medical transport support & 0.735 & 0.718 & & & 0.509 & 8.532 \\
\hline Combined neighborhood issues & -1.506 & 1.062 & 0.156 & 0.222 & 0.028 & \\
\hline \multicolumn{7}{|c|}{ Census Tract Level Variables } \\
\hline Census tract $>93.3 \%$ black $/ \mathrm{AA}$ & 0.110 & 0.582 & 0.849 & 1.117 & \multirow{5}{*}{\multicolumn{2}{|c|}{$\begin{array}{l}\text { Not generated } \\
\text { Not generated } \\
\text { Not generated } \\
\text { Not generated } \\
\text { Not generated }\end{array}$}} \\
\hline Census tract over $\$ 50,600 \mathrm{MAHI}$ & 0.402 & 0.787 & 0.610 & 1.495 & & \\
\hline Census tract $>80 \% \mathrm{HS}$ diploma & 1.112 & 0.676 & 0.101 & 3.039 & & \\
\hline Census tract $>20 \%$ Bachelor deg & -0.395 & 0.765 & 0.603 & 0.674 & & \\
\hline FF restaurants in top quintile & -0.285 & 0.382 & 0.453 & 0.750 & & \\
\hline
\end{tabular}


Table 13: $\quad$ List of Dissertation Hypotheses and Decision Rules

\begin{tabular}{|c|c|c|c|}
\hline ID & Null Hypotheses & Key Independent Variables & Decision Rule \\
\hline $\mathbf{A}$ & $\begin{array}{l}\text { Health stewardship factors do not significantly predict health } \\
\text { behaviors, health outcomes, or medical utilization (culture } \\
\text { hypothesis) }\end{array}$ & $\begin{array}{l}\text {-Church attendance }>1 \mathrm{x} / \text { week } \\
\text {-Prays frequently }>1 \mathrm{x} / \text { week } \\
\text {-Feel God's love for me }>1 \mathrm{x} / \text { day } \\
\text {-Religion helps greatly with stress } \\
\text {-Passive problem solving score }\end{array}$ & $\begin{array}{l}\text {-Reject null } \\
\text {-Do not reject null } \\
\text {-Do not reject null } \\
\text {-Do not reject null } \\
\text {-Reject null }\end{array}$ \\
\hline B & $\begin{array}{l}\text { Views of disease and the body do not significantly predict health } \\
\text { behaviors, health outcomes, or medical utilization (culture } \\
\text { hypothesis) }\end{array}$ & -Ever used home remedy & -Do not reject null \\
\hline $\mathbf{C}$ & $\begin{array}{l}\text { Interpersonal and social trust do not significantly predict health } \\
\text { behaviors, health outcomes, or medical utilization (historical } \\
\text { experience hypothesis) }\end{array}$ & -Trust in medical provider & -Do not reject null \\
\hline D & $\begin{array}{l}\text { Perceptions of provider quality or health care system ratings do } \\
\text { not significantly predict health behaviors, health outcomes, or } \\
\text { medical utilization (historical experience hypothesis) }\end{array}$ & $\begin{array}{l}\text {-Satisfaction with health care } \\
\text {-Unfair medical experience }\end{array}$ & $\begin{array}{l}\text {-Do not reject null } \\
\text {-Reject null }\end{array}$ \\
\hline $\mathbf{E}$ & $\begin{array}{l}\text { Proximity to and quality of primary care facilities does not } \\
\text { significantly predict health behaviors, health outcomes, or } \\
\text { medical utilization }\end{array}$ & -Difficulty reaching health services & -Do not reject null \\
\hline $\mathbf{F}$ & $\begin{array}{l}\text { Proximity to and quality of nutritious, quality food does not } \\
\text { significantly predict health behaviors, health outcomes, or } \\
\text { medical utilization (neighborhood hypothesis) }\end{array}$ & $\begin{array}{l}\text {-Serious problem: grocery access } \\
\text {-FF restaurants in top quintile }\end{array}$ & $\begin{array}{l}\text {-Do not reject null } \\
\text {-Reject null }\end{array}$ \\
\hline G & $\begin{array}{l}\text { Proximity to and quality of parks, fitness facilities, and built } \\
\text { environment do not significantly predict health behaviors, health } \\
\text { outcomes, or medical utilization (neighborhood hypothesis) }\end{array}$ & $\begin{array}{l}\text {-Serious problem: lack of parks } \\
\text {-Serious problem: poor sidewalks } \\
\text {-Serious problem: trash/litter } \\
\text {-Serious problem: heavy traffic } \\
\text {-Serious problem: excessive noise }\end{array}$ & $\begin{array}{l}\text {-Reject null } \\
\text {-Do not reject null } \\
\text {-Reject null } \\
\text {-Do not reject null } \\
\text {-Do not reject null }\end{array}$ \\
\hline $\mathbf{H}$ & $\begin{array}{l}\text { Proximity to and quality of social bonds do not significantly } \\
\text { predict health behaviors, health outcomes, or medical utilization } \\
\text { (social bonds hypothesis) }\end{array}$ & $\begin{array}{l}\text {-Low medical transport support } \\
\text {-Social network size: } 3+\text { friends } \\
\text {-Stress due to neighborhood crime }\end{array}$ & $\begin{array}{l}\text {-Do not reject null } \\
\text {-Reject null } \\
\text {-Reject null }\end{array}$ \\
\hline
\end{tabular}


hypotheses A, D, F, G, and H. Neighborhood factors (such as those postulated in hypotheses $\mathrm{F}, \mathrm{G}$, and $\mathrm{H}$ ) are shown to be significant factors in multiple analyses, thereby highlighting the impact of neighborhood structure via the nesting. Living in a census tract classified as one in the top quintile of fast food restaurant availability is implicated in diabetes status and possibly hypercholesterolemia.

However, other neighborhood factors, especially those encompassing perceptions of neighborhood problems were significant in an unexpected way. The role of vigilance as a component of high health stewardship was offered to explain these findings. Another reason that these findings are unexpected may be what the variables are capturing. In essence, the neighborhood problems variables measure participants' perceptions. The author proposes that if the variables relating to park availability or grocery store access were measured in a manner similar to fast food restaurants (i.e., by assessing how many are within a particular proximity in a neighborhood), then the variables would be statistically significant in the expected manner. In other words, while the perceptions of neighborhood measures are useful, empirical measures are also needed to demonstrate the impact of the proximity and quality of physician practices, pharmacies, grocery stores, and parks (including playgrounds, fitness facilities, and the built environment).

Social status emerged as a possible explanation for the significant predictors of hypertension status while various $\mathrm{M}^{3}$ Ecosocial Framework factors were significant predictors of diabetes status. This leads the author to propose that different diseases can be sensitive to different social determinants. Based on the analyses conducted in this dissertation, diabetes seems to be more sensitive to historical factors and neighborhood structure (e.g. fast food restaurant availability), while hypertension seems to be more sensitive to education attainment and neighborhood (possibly as a markers for social status). In other words, just as two different viruses or can cause two different diseases (e.g. HIV and the influenza), different social factors may contribute to increased risk for certain diseases but not others.

\section{Limitations}

There are several limitations in the analyses carried out in this research. First, this analyses and the $\mathrm{M}^{3}$ Ecosocial Framework were tested with a secondary dataset. Variables in the JHS were not developed to specifically test the author's hypotheses. In spite of this limitation, however, the Jackson Heart Study represents the best dataset that currently exists (of which the researcher is aware) to attempt to answer the research questions posed in this dissertation regarding the impact of health cosmology variables on health behaviors and ultimately cardiovascular disease.

Second, only some study participants were selected randomly. This may have introduced selection bias. Descriptive statistics reveal nearly $100 \%$ of JHS participants reported high trust their medical provider and high satisfaction with their health care. It is likely that JHS investigators were not able to recruit a sizable number of participants that did not trust their medical provider or were not satisfied with their health care. Thus, 
the results and the resulting analyses may not reflect a representative sample of African Americans with a high degree of mistrust and dissatisfaction in their medical provider and the health care system.

Third, several of the statistical models, especially ones for people living in least advantaged census tracts, have small sample sizes. The small sample sizes reduce the confidence that one should place in the results and subsequent analyses. This could not be avoided given the high number of variables (often 30 or more) included in the statistical models in order control for traditional explanatory variables and test the $\mathrm{M}^{3}$ Ecosocial Framework.

Fourth, the dissertation lacks mortality data. Mortality data for the JHS will not be available to researchers for several more months. The limitation that this imposes on this dissertation is that the author was unable to account for persons who may have died due to cardiovascular disease and compare outcomes based on hospitalization. 


\section{CHAPTER 5: DISCUSSION, IMPLICATIONS, AND TRANSFORMING THE HEALTH CARE SYSTEM IN THE UNITED STATES}

\section{Research and Policy Implications of Dissertation Findings}

The statistical analyses in this dissertation were conducted within a study sample that is solely comprised of African Americans. Thus, finding statistically significant differences within an exclusively African American sample is encouraging for future analyses between African American and European Americans or other ethnic/racial group combinations. If there are within-group ethnic differences, as demonstrated in this dissertation, there are likely to be between-group ethnic differences that are consistent with what is predicted in the $\mathrm{M}^{3}$ Ecosocial Framework.

In the multilevel statistical analyses reported in the previous chapter, modifiable factors were a large percentage of the significant predictors of physical activity and cardiovascular health outcomes. Behavioral determinants such as alcohol consumption and physical activity were consistently significant predictors of the hypertension, diabetes, and hypercholesterolemia. Health cosmology factors-such as religiosity, passive problem solving, and discrimination-were statistically significant predictors of the physical activity and diabetes.

The mutability of many of the significant predictors is an important finding since it highlights the fact that many of the determinants can be changed to help reduce the prevalence and incidence of CVD risk factors. For example, if physical activity was increased and experiences of discrimination (unfair medical treatment) were decreased in the populations characterized by health disparities, health outcomes would be greatly improved. The statistically significant impact of alcohol drinking in this dissertation is consistent with what is demonstrated in the literature. Encouraging African Americans to drink beneficial forms of alcohol (most likely red wine) in moderation can help reduce the incidence of diabetes and hypertension, thereby reducing the incidence of cardiovascular heart disease.

Evidence is also found to support the contention that diabetes development may be influenced by the fact that prevention of the disease may involve more medical interaction. Hypertension development, on the other hand, may be more sensitive to and impacted by indicators of social status. Therefore, devising efforts and interventions to reduce the incidence of diabetes and hypertension may involve different strategies to deal with some of the different factors leading to increased risk.

Descriptive statistics of the JHS sample reveal differences between African American women and men in terms of economic measures, cultural measures, and historical experiences measures. Although gender differences were not a focus of this dissertation, it is clear that an analysis gender differences is an important next step in terms of enhancing the explanatory power of the $\mathrm{M}^{3}$ Ecosocial Framework. The author conjectures that gender theories and woman studies will be valuable lenses through which 
to properly contextualize gender differences. After differences in gender are properly contextualized, interventions can then be crafted that will be better suited to reach African American females as a distinct population and African American males as another distinct population.

As stated in the last chapter, one of the major limitations of this dissertation research was the fact that data that were previously collected was used for the statistical analyses. Questions used to capture various measures were already written and collected in the home interviews or during the clinical exam. It is clear that certain questions could be much stronger or written in a way that would provide much needed detail. Future research should build on the questions used in the JHS and stronger, more precise questions should be developed.

One of the best methods to develop stronger and more precise questions is through qualitative research methods. Focus group research would be especially suited toward gaining information from participants, empowering participants with information, and encouraging dialogue between participants (Schwarz et al., 2000; Chandler \& Tolbert, 2003; Chiu, 2003; Ruff et al., 2005). Questions based on the $\mathrm{M}^{3}$ Ecosocial Framework can be constructed and presented to participants who can be stratified by disease status (i.e. people who have not been diagnosed with a CVD risk factor and people who have been diagnosed with a CVD risk factor) and neighborhood status (i.e. those living in a least advantaged census tract and those who live in a more advantaged census tract). The focus groups should also be stratified by gender in order to capture issues that may be specific to African American men or African American women. A strong qualitative research design would yield participants who will provide rich descriptions of their health behaviors and the way they deal with their health issues. Many of the variables that were not significant in this dissertation may in fact still play a key role, if a survey was designed based on an analysis of a well-designed qualitative study.

Equipped with sharper questions informed by the qualitative research conducted in the future, researchers could then build on and replicate the design of the Jackson Heart Study - perhaps in a city such as Memphis, Tennessee. Memphis is an ideal research target for such a study since it is a city that currently leads the nation in the rates of infant mortality, obesity, food insecurity, syphilis, gonorrhea, and chlamydia. Such a future research study would be the key to developing evidence-based interventions that can effectively increase the levels of physical activity, improve health behaviors (i.e. poor dietary patterns and risky sexual behaviors), and ultimately reduce risk for cardiovascular disease risk factors and sexually transmitted infections.

Infrastructure for such a research study exists or is being developed at the newly funded Consortium for Health Education, Economic Empowerment, and Research (CHEER) led by the University of Tennessee Health Science Center. CHEER partners (LeMoyne Owen College, Memphis-Shelby County Health Department, Memphis Housing Authority, First Baptist Church Lauderdale, and Mustard Seed Inc.) would all be key in engaging the community in recruitment and retention efforts. Qualitative research design and implementation could be conducted with skilled qualitative researchers at the 
University of Memphis and University of Tennessee Health Science Center. The MidSouth Survey Research Center personnel at the University of Memphis could handle the telephone administration component of the study. The geographic information science (GIS) expertise can be provided by faculty and students in the Earth Sciences department at the University of Memphis and supplemented with work being conducted by researchers at the Center for the Advancement of Youth Development at Rhodes College.

\section{Research and Policy Implications of the $\mathbf{M}^{3}$ Ecosocial Framework}

Although the findings in this dissertation represent a small first step in testing the Multilevel, Multicultural, and Multi-temporal Ecosocial Framework of Population Health, the results indicate that the $\mathrm{M}^{3}$ Ecosocial Framework may prove to be a paradigm-shift in the way we think about, treat, and analyze health and population health. The potential translation possibilities are many. Once researchers understand that different ethnic groups view the world of health and health care from different vantage points, future researchers can begin to outline and describe health cosmologies for other ethnic groups. As suggested by Blendon and colleagues (2008), differences in racial/ethnic groups' perceptions of quality in physician care in the US suggest that each racial/ethnic group may have their own particular health cosmologies. Globally, to provide an example, an understanding of the Maori, indigenous Australian, indigenous South African, and Dravidian view of the world of health and health care could help health providers design better ways of improving the health status for people in New Zealand, Australia, South Africa, and India.

In the United States, once differences in health cosmologies are understood by researchers, curricula can be modified so that health providers can begin to think about the ways in which adherence to treatment plans might be altered by someone's view of health and health care. Treatment plans can be designed that can account for differences in racial and ethnic populations' views of the world of health and health care. Policy makers and health care administrators could then formulate, implement, and evaluate social and health policy that can address key differences in perceptions of quality, medical trust, disease preferences, and health stewardship. It is expected that an accurate understanding and proper application of the $\mathrm{M}^{3}$ Ecosocial Framework can help reduce observed variations in health outcomes between racial and ethnic groups and close the gap in health disparities.

\section{Transforming the Health Care System in the United States}

Given this collective information, how do we proceed to effectively treat African American patients and reverse the tide of health disparities that plague the African American community? The first step is to acknowledge the disparities within the current health care system and understand that the health care system is inexpert, in its current form, to effectively treat this population. This is true not only because of continued bias 
and discrimination in health care, but because many African Americans possess a different view of the world of health and health care - due to differences in culture and historical experiences - compared to their European American counterparts and compared to the European American physicians who are disproportionately charged with their healthcare.

Secondly, we should understand that providing more training regarding cultural competence or provider empathy is not enough to eliminate health disparities. Research shows that even when providers profess to be egalitarian and enlightened in terms of treating people fairly regardless of race or ethnicity, biases often still exist on an unconscious level (Smedley et al., 2003; Dovidio et al., 2008). These biases then affect providers' treatment decisions in a way that adversely impacts the health outcomes of African Americans. In addition, cultural competence training often does not address the historical legacy of racism, which means that the role of institutional, interpersonal, and internalized racism remains hidden (Tyson, 2007).

This is a point that cannot be ignored. In their article "Beyond cultural competence: Critical consciousness, social justice, and multicultural education," Kumagai and Lypson (2009, p. 782) articulate the point more precisely. They state:

...we propose that educating physicians skilled at addressing the health care needs of a diverse society involves not the fulfillment of a competency as some sort of educational nirvana, but the development of an orientation-a critical consciousness - which places medicine in a social, cultural, and historical context and which is coupled with an active recognition of societal problems and a search for appropriate solutions.

The solution for the problem of health disparities must include not only educating individuals to develop a critical consciousness that contextualizes the existence of health disparities, but also include transforming the health care system - from health education training to delivery of health services. In order to address the multiple deficiencies of the current health care system in the United States, a health care system should be designed to be ethnically responsive and relevant at the local level in ways that correspond to the view of health and health care in the African American community.

What does it mean to create an ethnically responsive and relevant health care system for African Americans? Based on the statistical analyses of the Jackson Heart Study sample, it means attention should first be paid to preventive medicine that will help decrease new cases of diabetes, hypertension, and hypercholesterolemia. By reducing cardiovascular disease risk factors, the incidence of coronary heart disease, myocardial infarction, stroke, and other cardiovascular diseases will be reduced. Preventive medicine efforts should focus on boosting physical activity levels in the community in order to lose weight and encouraging non-alcoholic persons to drink alcohol in moderation. The statistical significance of car ownership and income in the total physical activity score model suggests that access to parks and fitness facilities may be potential barriers to people getting appropriate exercise. This means preventive medicine efforts 
should also include addressing neighborhood structure involving the access to places where people can exercise.

Next, creating an ethnically responsive and relevant health care system for African Americans nationwide means addressing factors involved in the treatment of disease. Policy initiatives are needed to dramatically increase the numbers of African American health care providers to improve perceptions of quality and increase levels of interpersonal and social trust. African Americans are underrepresented in the medical professions (Council on Graduate Medical Education, 2005) and this greatly limits the current health care system's ability to be ethnically responsive and relevant to the African American community. It also means having health care providers who are comfortable and conversant with a spiritual perspective of medicine, not only a biological perspective of medicine. Creating an ethically responsive and relevant health care system also means greatly increasing the profile, role, and funding levels of the National Institute for Minority Health and Health Disparities and the national Office of Minority Health to increase the resources available to health disparities researchers and to fund interventions that prove to be successful in reducing health disparities.

Creating an ethnically responsive and relevant health care system also means training and involving African American churches, pastors, and lay members in a conversation about adopting a perspective that promotes individual and communal health stewardship. Individual health stewardship is defined as taking personal responsibility for the multiple behavioral determinants of health - whether it is improving dietary intake, increasing and maintaining a regular exercise regimen, and avoiding unhealthy habits such as smoking (all under the guidance of a physician). Communal health stewardship is defined as people in a community taking collective responsibility for the multiple behavioral determinants of health, by improving access to and quality of health-generating facilities (e.g. grocery stores with fresh fruits and vegetables, parks and fitness facilities, etc.) and reducing access to health-negating facilities (e.g. pollution sites, an oversupply of fast food restaurants, liquor stores, etc.). The statistical results from the Jackson Heart Study sample highlighted the statistically significant impact of living in a neighborhood with a high amount of fast food restaurants. Communal health stewardship means addressing the impact of living in neighborhoods where fresh fruits and vegetables may not be accessible or affordable.

To a large degree, this means modifying and incorporating a theological perspective of holistic salvation in African American churches-namely, that church members should be saved not only from spiritual and physical sin, but also from a disproportionate burden of sickness and premature death. Vigilance should be promoted as a component of health stewardship as well, especially in the least advantaged neighborhoods.

Pastors should preach and members should be encouraged to practice a theological perspective that encourages individual and communal health stewardship along with vigilance. Creating an ethnically responsive and relevant health care system means starting and maintaining viable and sustainable church health ministries in a large percentage of African American churches. Health ministries would serve as a bridge to 
the local public health system - utilizing mobile health units, employing lay community health workers, coordinating screenings for chronic diseases and sexually transmitted infections, discussing health issues and determinants of health, and coaching members on how to start and maintain a healthy lifestyle.

It is also recognized that a large percentage of African Americans do not attend church. Therefore, a media campaign should be designed to promote individual and communal health stewardship in the broader African American community, with messages tailored for television, radio, print and the Internet. In order to obtain the desired effect, individuals must understand the importance of the information being provided and it must be effectively communicated in order to invoke personal application. Media campaigns allow for "change in health behavior across multiple levels of human experience - from the individual to the community" (Finnegan \& Viswanath, 2002, p. 364). Furthermore, "media institutions play a crucial role in health behavior change because they are key gatekeepers for disseminating information in social systems and because, as socializing agents, they have powerful effect on legitimizing social norms of behavior; therefore, they can and should be used to create positive impact in public health" (Finnegan \& Viswanath, 2002, p. 364).

Hip hop culture is a dominant force in African American youth culture and can be a major component of a media campaign designed to educate and change behavior in young persons of African descent. Dr. Rani Whitfield —otherwise known as the Hip Hop Doc - is a medical doctor from Baton Rouge, Louisiana who successfully utilizes the art form of hip-hop to engage young people (including young adults who have grown up on hip hop). On his website, Dr. Whitfield conducts a video interview with popular rapper Lil' Boosie concerning his diabetes and how he successfully manages the condition. In another instance, the Memphis group Connect to Protect (a coalition of organizations and individuals working to reduce HIV among African American females) hosted a concert in December 2009 featuring Memphis rapper Yo' Gotti where HIV testing was conducted on the site of the concert. These highly innovative approaches utilize hip hop artists and appropriate hip hop artforms (i.e. actually rapping about health education in the case of Dr. Rani Whitfield) in order to facilitate health education among African American youth whose health concerns are often peripheral in American society.

An ethnically responsive and relevant health care system should be locally flexible and persistently proactive - not reactive. Well-funded mobile health units staffed with highly trained and ethnically responsive health providers should be used to help bring medical and pharmaceutical services to areas where people have transportation issues and as a result may be unwilling or less likely to visit a physician. The existing health care system requires the patient to travel to the health provider and by the time the patient does so, the disease has often reached a more deadly and costly phase. In a proactive health care system, the health provider will go to the patient and meet the patient where the patient lives, works, and recreates. This approach will include examples of the "anticipatory care model" and other programs that reach into the community (Williams et al., 2008). 
If the existing health care system is transformed and adopts an ethnically responsive and relevant, locally flexible, and persistently proactive approach, our health care system can increase patient satisfaction and trust, increase individual and communal health stewardship, improve health outcomes, and ultimately reduce the cost of health care in the United States. This approach moves us in the direction of eliminating health disparities, thereby improving African American life expectancy and reducing disproportionate morbidity and early mortality among African Americans.

An ethnically responsive and relevant health care system does not mean a return to separate and unequal - it represents a move towards meeting and addressing the unique needs of the African American community when funded properly and given vigorous attention and energy on the nati onal, state, and local levels. This ethnically responsive and relevant health care system should be primarily funded by the federal government, preferably in conjunction with a universal health care system, or at least with increased access among the national population, and supported by state governments, local governments, private enterprises, and nonprofit organizations, even African American churches.

Performance-based pay and bonuses for health care providers in a ethnically responsive and relevant health care system would be based on indicators such as improving patient satisfaction and trust, increasing communal and individual health stewardship, improving health outcomes, and reducing preventable and costly hospitalizations in a geographic region for ambulatory care sensitive conditions (i.e. diabetes, hypertension, heart disease, asthma, etc.). Additionally, reimbursements from programs such as Medicaid should be made in a much more timely fashion to health care providers. Many African American health providers serve disproportionately poor populations and cannot sustain viable private practices with low and late Medicaid reimbursements. In order to make the health care system locally flexible, much more resources should be directed to these providers to assist them in providing their services in ways that are more amenable to the needs of the communities in which they serve. Furthermore, they should have a prominent role in designing interventions and programs that can improve health outcomes.

Finally, an ethnically responsive and relevant health care system designed to meet the needs of the African American population is desperately needed for the dire situation faced in the African American community. Unfortunately, the current health care system that African Americans look to for hope and healing is plagued with a long legacy of medical apartheid and an intransigence to confront the past and current activities that create the very soil in which health disparities grow and thrive. New wine should not be poured into old wineskins, but if we augment the current health care system with one that is locally appropriate and ethnically responsive and relevant to the health care needs of the African American population, we can possibly heal both our patients and the current system - and make them whole. 


\section{LIST OF REFERENCES}

Abrams, L. S., \& Stormer, C. C. (2002). Sociocultural variations in the body image perceptions of urban adolescent females. Journal of Youth and Adolescence , 31(6), $443-450$.

Abrums, M. (2000). "Jesus will fix it after awhile": Meanings and health. Social Science and Medicine, 50(1), 89-105.

Ahmed, A. T., Mohammed, S. A., \& Williams, D. R. (2007). Racial discrimination \& health: Pathways \& evidence. The Indian Journal of Medical Research, 126(4), 318327.

Altschuler, A., Somkin, C. P., \& Adler, N. E. (2004). Local services and amenities, neighborhood social capital, and health. Social Science and Medicine , 59(6), 12191229 .

Andersen, R. M. (1995). Revisiting the behavioral model and access to medical care: Does it matter? Journal of Health and Social Behavior, 36(1), 1-10.

Ansari, Z. (2007). The concept and usefulness of ambulatory care sensitive conditions as indicators of quality and access to primary health care. Australian Journal of Primary Health, 13(3), 91-110.

Anselin, L., McCann M., \& Kho Y. (2009). OpenGeoDa, Open Source Software for Exploratory Spatial Data Analysis , GeoDa Center for Geospatial Analysis and Computation, Arizona State University.

Armstrong, K., McMurphy, S., Dean, L. T., Micco, E., Putt, M., Halbert, C. H., et al. (2008). Differences in the patterns of health care system distrust between blacks and whites. Journal of General Internal Medicine, 23(6), 827-833.

Armstrong, K., Ravenell, K. L., McMurphy, S., \& Putt, M. (2007). Racial/ethnic differences in physician distrust in the United States. American Journal of Public Health, 97(7), 1283-9.

Aruffo, J. F., Coverdale, J. H., Pavlik, V. N., \& Vallbona, C. (1993). AIDS knowledge in minorities: Significance of locus of control. American Journal of Preventive Medicine, 9(1), 15-20.

Avis, N. E., Ory, M., Matthews, K. A., Schocken, M., Bromberger, J., \& Colvin, A. (2003). Health-Related quality of life in a multiethnic sample of middle-aged women: Study of women's health across the nation (SWAN). Medical Care, 4l(11), 12621276. 
Ayalon, L., \& Young, M. A. (2005). Racial group differences in help-seeking behaviors. The Journal of Social Psychology, 145(4), 391-404.

Baker, R. B., Washington, H. A., Olakanmi, O., Savitt, T. L., Jacobs, E. A., Hoover, E., \& Williams, D. R. (2009). Creating a segregated medical profession: African American physicians and organized medicine, 1846-1910. Journal of the National Medical Association, 101(6), 501-512.

Bamshad, M. J., Wooding, S., Watkins, W. S., Ostler, C. T., Batzer, M. A., \& Jorde, L. B. (2003). Human population genetic structure and inference of group membership. American Journal of Human Genetics, 72(3), 578-589.

Barbujani, G., Magagni, A., Minch, E., \& Cavalli-Sforza, L. L. (1997). An apportionment of human DNA diversity. Proceedings of the National Ac ademy of Sciences of the United States of America, 94(9), 4516-4519.

Barnett, A. G., Williams, G. M., Schwartz, J., Best, T. L., Neller, A. H., Petroeschevsky, A. L., \& Simpson, R. W. (2006). The effects of air pollution on hospitalizations for cardiovascular disease in elderly people in Australian and New Zealand cities. Environmental Health Perspectives, 114(7), 1018-1023.

Barroso, J., McMillan, S., Casey, L., Gibson, W., Kaminski, G., \& Meyer, J. (2000). Comparison between African-American and white women in their beliefs about breast cancer and their health locus of control. Cancer Nursing, 23(4), 268-276.

Beaulac, J., Kristjansson, E., \& Cummins, S. (2009). A systematic review of food deserts, 1966-2007. Preventing Chronic Disease, 6(3), A105.

Bekhuis, T., Cook, H., Holt, K., Scott-Lennox, J., Lennox, R., Price, L., \& Fryer, J. G. (1995). Ethnicity, church affiliation and beliefs about the causal agents of health: A comparative study employing a multivariate analysis of covariance. Health Education Research, 10(1), 73-82.

Billings, J. (2003). Appendix B: Ambulatory care sensitive conditions. In Tools for Monitoring the Health Care Safety Net. AHRQ Publication No. 03-0027, September 2003. Agency for Healthcare Research and Quality, Rockville, MD. Accessed July 30, 2008 from: http://ftp.ahrq.gov/data/safetynet/billappb.htm.

Blendon, R. J., Buhr, T., Cassidy, E. F., Perez, D. J., Hunt, K. A., Fleischfresser, C., \& Herrmann, M. J. (2007). Disparities in health: Perspectives of a multi-ethnic, multiracial America. Health Affairs (Project Hope), 26(5), 1437-1447.

Blendon, R. J., Buhr, T., Cassidy, E. F., Pérez, D. J., Sussman, T., Benson, J. M., \& Herrmann, M. J. (2008). Disparities in physician care: Experiences and perceptions of a multi-ethnic America. Health Affairs (Project Hope), 27(2), 507-517. 
Bogart, L. M., \& Thorburn, S. (2005). Are HIV/AIDS conspiracy beliefs a barrier to HIV prevention among African Americans? Journal of Acquired Immune Deficiency Syndromes, 38(2), 213-218.

Boulware, L. E., Cooper, L. A., Ratner, L. E., LaVeist, T. A., \& Powe, N. R. (2003). Race and trust in the health care system. Public Health Reports, 118(4), 358-365.

Bourjolly, J. N. (1999). Locus of control among black and white women with breast cancer. Journal of Psychosocial Oncology, 17(1), 21-31.

Boyd-Franklin, N., \& Bry, B. H. (2000). Reaching Out in Family Therapy: Home-Based, School, and Community Interventions. New York: The Guilford Press.

Brewster, K., Wileyto, E. P., Kessler, L., Collier, A., Weathers, B., Stopfer, J. E., et al. (2007). Sociocultural predictors of breast cancer risk perceptions in African American breast cancer survivors. Cancer Epidemiology, Biomarkers and Prevention: A Publica tion of th e American Associa tion for Cancer Resear ch, Cosponsored by the American Society of Preventive Oncology, 16(2), 244-248.

Buchwald, D., Manson, S. M., Pearlman, T., Umali, J., \& Kith, P. (1996). Race and ethnicity in patients with chronic fatigue. Journal of Chronic Fatigue Syndrome , 2(1), 53-66.

Burns, M. J., \& Dillon, F. R. (2005). AIDS health locus of control, self-efficacy for safer sexual practices, and future time orientation as predictors of condom use in African American college students. Journal of Black Psychology, 31(2), 172-188.

Carpenter, W. R., Godley, P. A., Clark, J. A., Talcott, J. A., Finnegan, T., Mishel, M., et al. (2009). Racial differences in trust and regular source of patient care and the implications for prostate cancer screening use. Cancer, 115(21), 5048-5059.

Chandler, D., \& Torbert, B. (2003). Transforming inquiry and action: Interweaving 27 flavors of action research. Action Research, 1(2), 133-152.

Chen, F. M., Fryer, G. E., Phillips, R. L., Wilson, E., \& Pathman, D. E. (2005). Patients' beliefs about racism, preferences for physician race, and satisfaction with care. Annals of Family Medicine, 3(2), 138-143.

Chiu, L. F. (2003). Transformational potential of focus group practice in participatory action research. Action Research, 1(2), 165-183.

Congdon, P. (2006). Modelling multiple hospital outcomes: The impact of small area and primary care practice variation. International Journal of Health Geographics, 5, 50.

Corbie-Smith, G., Thomas, S. B., \& St George, D. M. M. (2002). Distrust, race, and research. Archives of Internal Medicine, 162(21), 2458-2463. 
Council on Graduate Medical Education. (2005). Minorities in Medicine: An Ethnic and Cultural Challenge for Phys ician Training-An Update. Rockville, MD: Council on Graduate Medical Education, Health Resources and Services Administration, US Department of Health and Human Services.

Cromley, E. K., \& McLafferty, S. (2002). GIS and Public Health. New York: The Guilford Press.

Cykert, S., Joines, J. D., Kissling, G., \& Hansen, C. J. (1999). Racial differences in patients' perceptions of debilitated health states. Journal of General Internal Medicine, 14(4), 217-222.

Doescher, M. P., Saver, B. G., Franks, P., \& Fiscella, K. (2000). Racial and ethnic disparities in perceptions of physician style and trust. Archives of Family Med icine, 9(10), 1156-1163.

Dovidio, J. F., Penner, L. A., Albrecht, T. L., Norton, W. E., Gaertner, S. L., \& Shelton, J. N. (2008). Disparities and distrust: The implications of psychological processes for understanding racial disparities in health and health care. Social Science and Medicine, 67(3), 478-486.

Dressler, W. W., Oths, K. S., \& Gravlee, C. C. (2005). Race and ethnicity in public health research: Models to explain health disparities. Annual Review of Anthropology , 34(1), 231-252.

Drummond, M. F., Sculpher, M. J., Torrance, G. W., O'brien, B. J., \& Stoddart, G. L. (2005). Methods for the Economic Evaluatio $n$ of Health Care Programmes. New York: Oxford University Press.

Du Bois, W. E. B. The Souls of Black Folk. 1903. New York: Bantam.

Elliott, P., \& Wartenberg, D. (2004). Spatial epidemiology: Current approaches and future challenges. Environmental Health Perspectives, 112(9), 998-1006.

Elsevier Science Publishers. (2004). Scopus. New York: Elsevier.

Environmental Systems Research Institute (2006). ArcView 9.2 . Redlands, CA: Environmental Systems Research Institute.

Evans, R. G., Barer, M. L., \& Marmor, T. R. (1994). Why are Some People Healthy and Others Not?: The Determinants of Health of Populations. New York: Aldine de Gruyter.

Fiori, K. L., Hays, J. C., \& Meador, K. G. (2004). Spiritual turning points and perceived control over the life course. International Journal of Aging and Human Development, 59(4), 391-420. 
Finnegan J. R. \& Viswanath K. (2002). Communication theory and health behavior change: the media studies framework. In Glanz K., Lewis F. M., Rimer, B. K. (Eds.). Health Behavior and Health Education: Theory, Research, and Practice. 3rd ed. San Francisco: Jossey-Bass.

Fiscella, K., \& Williams, D. R. (2004). Health disparities based on socioeconomic inequities: Implications for urban health care. Academic Medicine: Journal of the Association of American Medical Colleges, 79(12), 1139-1147.

Fongwa, M. N., Cunningham, W., Weech-Maldonado, R., Gutierrez, P. R., \& Hays, R. D. (2008). Reports and ratings of care: Black and white medicare enrollees. Journal of Health Care for the Poor and Underserved, 19(4), 1136-1147.

Ford, M. E., \& Kelly, P. A. (2005). Conceptualizing and categorizing race and ethnicity in health services research. Health Services Research, 40(5 Pt 2), 1658-1675.

Fu, A. Z., \& Kattan, M. W. (2006). Racial and ethnic differences in preference-based health status measure. Current Medical Research and Opinion, 22(12), 2439-2448.

Galanos, A. N., Strauss, R. P., \& Pieper, C. F. (1994). Sociodemographic correlates of health beliefs among black and white community dwelling elderly individuals. International Journal of Aging and Human Development, 38(4), 339-350.

Gaskin, D. J., \& Frick, K. D. (2008). Race and ethnic disparities in valuing health. Medical Decision Making: An International Journal of the Society for Medical Decision Making, 28(1), 12-20.

Gaskin, D. J., Headen, A. E., \& White-Means, S. I. (2005). Racial disparities in health and wealth: The effects of slavery and past discrimination. The Review of Black Political Economy, 32(3), 95-110.

Gish, O. (1979). The political economy of primary care and "health by the people": An historical explanation. Issue: A Journal of Opinion, 9(3), 6-13.

Goodman, A. H. (2000). Why genes don't count (for racial differences in health). American Journal of Public Health, 90(11), 1699-1702.

Grubesic, T. H., \& Matisziw, T. C. (2006). On the use of ZIP codes and ZIP code tabulation areas (zctas) for the spatial analysis of epidemiological data. International Journal of Health Geographics, 5, 58.

Guadagnolo, B. A., Cina, K., Helbig, P., Molloy, K., Reiner, M., Cook, E. F., \& Petereit, D. G. (2009). Medical mistrust and less satisfaction with health care among Native Americans presenting for cancer treatment. Journal of Health Care for the Poor and Underserved, 20(1), 210-226. 
Guagliardo, M. F. (2004). Spatial accessibility of primary care: Concepts, methods and challenges. International Journal of Health Geographics, 3, 3.

Halbert, C. H., Armstrong, K., Gandy, O. H., \& Shaker, L. (2006). Racial differences in trust in health care providers. Archives of Internal Medicine, 166(8), 896-901.

Halbert, C. H., Weathers, B., Delmoor, E., Mahler, B., Coyne, J., Thompson, H. S., \& Lee, D. (2009). Racial differences in medical mistrust among men diagnosed with prostate cancer. Cancer, 115(11), 2553-2561.

Hall, D. E. (2006). Religious attendance: More cost-effective than Lipitor? Journal of the American Board of Family Medicine, 19(2), 103-109.

Hall, M. A. (2006). Researching medical trust in the United States. Journal of Health Organisation and Management, 20(5), 456-467.

Hausmann, L. R., Jeong, K., Bost, J. E., \& Ibrahim, S. A. (2008). Perceived discrimination in health care and health status in a racially diverse sample. Medical Care, 46(9), 905-914.

Heath, A. C., Madden, P. A., Grant, J. D., McLaughlin, T. L., Todorov, A. A., \& Bucholz, K. K. (1999). Resiliency factors protecting against teenage alcohol use and smoking: Influences of religion, religious involvement and values, and ethnicity in the Missouri adolescent female twin study. Twin Research: The Official Journal of the International Society for Twin Studies, 2(2), 145-155.

Herskovits, M. J. (1958). The Myth of the Negro Past. Boston: Beacon.

Hughes Halbert, C., Barg, F. K., Weathers, B., Delmoor, E., Coyne, J., Wileyto, E. P., et al. (2007). Differences in cultural beliefs and values among African American and European American men with prostate cancer. Cancer Control: Journal of the Moffitt Cancer Center, 14(3), 277-284.

ISI ResearchSoft. (2004). Reference Manager 11. Berkeley, California: ISI ResearchSoft.

Jackson Heart Study (JHS). (2003). Jackson Heart Study Protocol, Manual 1 . Accessed January 5, 2010 from:

http://jhs.jsums.edu/jhsinfo/Portals/0/pdf/manuals1/Manual1_general_description .pdf.

Jackson Heart Study (JHS) website. (2008). Study Description for Researchers. Accessed December 1, 2009 from:

http://jhs.jsums.edu/jhsinfo/Researchers/tabid/122/Default.aspx. 
Johnson, R. L., Saha, S., Arbelaez, J. J., Beach, M. C., \& Cooper, L. A. (2004). Racial and ethnic differences in patient perceptions of bias and cultural competence in health care. Journal of General Internal Medicine, 19(2), 101-110.

Jones, C. P. (2001). Invited commentary: "Race," racism, and the practice of epidemiology. American Journal of Epidemiology, 154(4), 299-304.

Jones, C. P., Truman, B. I., Elam-Evans, L. D., Jones, C. A., Jones, C. Y., Jiles, R., et al. (2008). Using "socially assigned race" to probe white advantages in health status. Ethnicity and Disease, 18(4), 496-504.

Jones, J. M. (2002). Toward a cultural psychology of African Americans. In W. J. Lonner, D. L. Dinnel, S. A. Hayes, \& D. N. Sattler (Eds.), Online Readings in Psychology and Culture (Unit 3, Chapter 1), (http://orpc.iaccp.org), Center for CrossCultural Research, Western Washington University, Bellingham, Washington USA.

Jorde, L. B., Watkins, W. S., Bamshad, M. J., Dixon, M. E., Ricker, C. E., Seielstad, M. T., \& Batzer, M. A. (2000). The distribution of human genetic diversity: A comparison of mitochondrial, autosomal, and y-chromosome data. American Journal of Human Genetics, 66(3), 979-988.

Kaprio, J. (2000). Science, medicine, and the future: Genetic epidemiology. British Medical Journal, 320(7244), 1257.

Kawachi, I., \& Berkman, L. F. (2003). Neighborhoods and Health. Oxford University Press, USA.

Kennedy, B. R., Mathis, C. C., \& Woods, A. K. (2007). African Americans and their distrust of the health care system: Healthcare for diverse populations. Journal of Cultural Diversity, 14(2), 56-60.

Kindig, D., \& Stoddart, G. (2003). What is population health? American Journal of Public Health, 93(3), 380-383.

Kindig, D. A. (2007). Understanding population health terminology. Milbank Quarterly, 85(1), 139-161.

King, G., \& Williams, D. R. (1995). Race and health: A multidimensional approach to African-American health. In Society and Health. (pp. 93-130). Oxford University Press, USA.

King, S. V., Burgess, E. O., Akinyela, M., Counts-Spriggs, M., \& Parker, N. (2005). Your body is god's temple: the spiritualization of health beliefs in multigenerational African American families. Research on Aging, 27(4), 420-446. 
Korthuis, P. T., Saha, S., Fleishman, J. A., McGrath, M. M., Josephs, J. S., Moore, R. D., et al. (2008). Impact of patient race on patient experiences of access and communication in HIV care. Journal of General Internal Medicine , 23(12), 20462052.

Kouznetsova, M., Huang, X., Ma, J., Lessner, L., \& Carpenter, D. O. (2007). Increased rate of hospitalization for diabetes and residential proximity of hazardous waste sites. Environmental Health Perspectives, 115(1), 75-79.

Krieger, N. (2001). Theories for social epidemiology in the 21 st century: An ecosocial perspective. International Journal of Epidemiology, 30(4), 668-677.

Kumagai, A. K., \& Lypson, M. L. (2009). Beyond cultural competence: Critical consciousness, social justice, and multicultural education. Academic Medicine: Journal of the Association of American Medical Colleges, 84(6), 782-787.

Kuppermann, M., Nease Jr, R. F., Gates, E., Learman, L. A., Blumberg, B., Gildengorin, V., \& Washington, A. E. (2004). How do women of diverse backgrounds value prenatal testing outcomes? Prenatal Diagnosis, 24(6), 424-429.

Kwate, N. O., Yau, C. Y., Loh, J. M., \& Williams, D. (2009). Inequality in obesigenic environments: Fast food density in New York City. Health and Place, 15(1), 364373.

LaVeist, T. A., Isaac, L. A., \& Williams, K. P. (2009). Mistrust of health care organizations is associated with underutilization of health services. Health Services Research, 44(6), 2093-2105.

Lee, J. S., Tamayo-Sarver, J., Kinneer, P., \& Hobgood, C. (2008). Association between patient race/ethnicity and perceived interpersonal aspects of care in the emergency department. Journal of the National Medical Association, 100(1), 79-85.

Levine, R., Briggs, N. C., \& Husaini, B. A. (2005). Geographic studies on black-white mortality. In Multicultural Medicine and Health Dis parities: Essentials for Clinical Practice. (pp. 33-50). New York: McGraw-Hill Professional.

Lin, S. S., \& Kelsey, J. L. (2000). Use of race and ethnicity in epidemiologic research: Concepts, methodological issues, and suggestions for research. Epidemiologic Reviews, 22(2), 187-202.

Lincoln, C. E., \& Mamiya, L. H. (1990). The Black Church in the African -American Experience. $13^{\text {th }}$ ed. Durham: Duke University Press.

Lloyd, C., Smith, J., \& Weinger, K. (2005). Stress and diabetes: A review of the links. Diabetes Spectrum, 18(2), 121-127. 
Lubetkin, E. I., Jia, H., Franks, P., \& Gold, M. R. (2005). Relationship among sociodemographic factors, clinical conditions, and health-related quality of life: Examining the EQ-5D in the U.S. General population. Quality of Life Research: A $n$ International Journal of Quality of Li fe Aspects o fTreatment, Care and Rehabilitation, 14(10), 2187-2196.

Luke, D. A. (2005). Multilevel Modeling. Thousand Oaks, CA: Sage.

Luo, N., Wang, Q., Feeny, D., Chen, G., Li, S. C., \& Thumboo, J. (2007). Measuring health preferences for health utilities index mark 3 health states: A study of feasibility and preference differences among ethnic groups in Singapore. Medical Decision Making: An International Journal of the Society fo $r$ Medical Decision Making, 27(1), 61-70.

Malcarne, V. L., Fernandez, S., \& Flores, L. (2005). Factorial validity of the multidimensional health locus of control scales for three American ethnic groups. Journal of Health Psychology, 10(5), 657-667.

Mansfield, C. J., Mitchell, J., \& King, D. E. (2002). The doctor as God's mechanic?: Beliefs in the southeastern United States. Social Science and Medicine , 54(3), 399409.

Mayo, R. M., Sherrill, W. W., Sundareswaran, P., \& Crew, L. (2007). Attitudes and perceptions of Hispanic patients and health care providers in the treatment of Hispanic patients: A review of the literature. Hispanic Health Care International , 5(2), 64-72.

Mays, V. M., Cochran, S. D., \& Barnes, N. W. (2007). Race, race-based discrimination, and health outcomes among African Americans. Annual Review of Psychology, 58, 201-225.

McCullough, M. E., Hoyt, W. T., Larson, D. B., Koenig, H. G., \& Thoresen, C. (2000). Religious involvement and mortality: A meta-analytic review. Health Psychology: Official Jou rnal of the Division o fHealth Ps ychology, A merican Ps ychological Association, 19(3), 211-222.

McElroy, A. M. (1996). Medical anthropology. In D. L. Levinson, M. E. Ember, \& H. R. A. Files (Eds.), Encyclopedia of Cultural Anthropology. New York: Henry Holt and Co.

McLaughlin, C. G., Normolle, D. P., Wolfe, R. A., McMahon, L. F., \& Griffith, J. R. (1989). Small-area variation in hospital discharge rates. Do socioeconomic variables matter? Medical Care, 27(5), 507-521.

Merriam-Webster Online Dictionary. (2010). Cosmology. In Merriam-Webster. Accessed March 13, 2010, from: http://www.merriam-webster.com/dictionary/cosmology. 
Minority Health and Health Disparities Research and Education Act of 2000. Pub. L. No. 106-525. 114 Stat. 2495 (2000).

Moore, L. V., \& Diez Roux, A. V. (2006). Associations of neighborhood characteristics with the location and type of food stores. American Journal of Public Health, 96(2), 325-331.

Morland, K., Wing, S., Diez Roux, A., \& Poole, C. (2002). Neighborhood characteristics associated with the location of food stores and food service places. American Journal of Preventive Medicine, 22(1), 23-29.

Musa, D., Schulz, R., Harris, R., Silverman, M., \& Thomas, S. B. (2009). Trust in the health care system and the use of preventive health services by older black and white adults. American Journal of Public Health, 99(7), 1293-1299.

National Center for Biotechnology Information (U.S.), National Library of Medicine (U.S.), \& National Institutes of Health (U.S.). (1996). PubMed. Bethesda, Md.: NCBI.

Neighbors, H. W., Jackson, J. S., Broman, C., \& Thompson, E. (1996). Racism and the mental health of African Americans: The role of self and system blame. Ethnicity and Disease, 6(1-2), 167-175.

Ngui, E. M., \& Flores, G. (2006). Satisfaction with care and ease of using health care services among parents of children with special health care needs: The roles of race/ethnicity, insurance, language, and adequacy of family-centered care. Pediatrics, 117(4), 1184-1196.

Outram, S. M., \& Ellison, G. T. (2006). Anthropological insights into the use of race/ethnicity to explore genetic contributions to disparities in health. Journal of Biosocial Science, 38(1), 83-102.

Oyana, T. J., \& Rivers, P. A. (2005). Geographic variations of childhood asthma hospitalization and outpatient visits and proximity to ambient pollution sources at a U.S.-Canada border crossing. International Journal of Health Geographics, 4, 14.

Oyserman, D., Uskul, A. K., Yoder, N., Nesse, R. M., \& Williams, D. R. (2007). Unfair treatment and self-regulatory focus. Journal of Experimental Social Psychology , 43(3), 505-512.

Ozdenerol, E., Williams, B. L., Kang, S. Y., \& Magsumbol, M. S. (2005). Comparison of spatial scan statistic and spatial filtering in estimating low birth weight clusters. International Journal of Health Geographics, 4, 19.

Ozdenerol, E. (2007). GIS and Community Health (Powerpoint). Accessed July 29, 2008 from: http://des.memphis.edu/esra/Teaching/Geog6613/Geog6613.htm. 
Paradies, Y. (2006). A systematic review of empirical research on self-reported racism and health. International Journal of Epidemiology, 35(4), 888-901.

Pascoe, E. A., \& Smart Richman, L. (2009). Perceived discrimination and health: A meta-analytic review. Psychological Bulletin, 135(4), 531-554.

Patton, M. Q. (2002). Qualitative Research a nd Evaluation Methods. Thousand Oaks, CA: Sage.

Payne, T. J., Wyatt, S. B., Mosley, T. H., Dubbert, P. M., Guiterrez-Mohammed, M. L., Calvin, R. L., et al. (2005). Sociocultural methods in the Jackson Heart Study: Conceptual and descriptive overview. Ethnicity and Disease, 15(4 Suppl 6), S6-3848.

Pearce, N., Foliaki, S., Sporle, A., \& Cunningham, C. (2004). Genetics, race, ethnicity, and health. British Medical Journal, 328(7447), 1070-1072.

Pearson, J. A. (2008). Can't buy me whiteness: New lessons from the titanic on race, ethnicity, and health. Du Bois Review: Social S cience Research on Race, 5(01), 2747.

Phillips Jr, R. L., Kinman, E. L., Schnitzer, P. G., Lindbloom, E. J., \& Ewigman, B. (2000). Using geographic information systems to understand health care access. Archives of Family Medicine, 9(10), 971-978.

Pietila, A. (2010). Not in My Neighborhood: How Bi gotry Shaped a Great American City. Chicago: Ivan R. Dee.

Piro, F. N., Næss, O., \& Claussen, B. (2007). Area deprivation and its association with health in a cross-sectional study: Are the results biased by recent migration? International Journal for Equity in Health, 6(1), 10.

Powell, L. M., Chaloupka, F. J., \& Bao, Y. (2007). The availability of fast-food and fullservice restaurants in the United States: Associations with neighborhood characteristics. American Journal of Preventive Medicine, 33(4 Suppl), S240-S245.

Race, Ethnicity, and Genetics Working Group. (2005). The use of racial, ethnic, and ancestral categories in human genetics research. American Journal of Human Genetics, 77(4), 519-532.

Rajakumar, K., Thomas, S. B., Musa, D., Almario, D., \& Garza, M. A. (2009). Racial differences in parents' distrust of medicine and research. Archives of Pediatrics and Adolescent Medicine, 163(2), 108-114.

Randall, V. R. (2006). Dying while Black. Dayton: Seven Principles Press. 
Rhone, S. N. (2006). Eating, Body Satisfaction, Ethnicity, and Women's Relationship with God. Thesis, Georgia State University.

Richman, L. S., Kohn-Wood, L. P., \& Williams, D. R. (2007). The role of discrimination and racial identity for mental health service utilization. Journal of Social and Clinical Psychology, 26(8), 960-981.

Roberts, J. S., Connell, C. M., Cisewski, D., Hipps, Y. G., Demissie, S., \& Green, R. C. (2003). Differences between African Americans and whites in their perceptions of Alzheimer disease. Alzheimer Disease and Associated Disorders, 17(1), 19-26.

Rosen, A. B., Tsai, J. S., \& Downs, S. M. (2003). Variations in risk attitude across race, gender, and education. Medical Decision Making: An In ternational Journal of the Society for Medical Decision Making, 23(6), 511-517.

Rosenberg, N. A., Pritchard, J. K., Weber, J. L., Cann, H. M., Kidd, K. K., Zhivotovsky, L. A., \& Feldman, M. W. (2002). Genetic structure of human populations. Science, 298(5602), 2381-2384.

Royal, C. D., \& Dunston, G. M. (2004). Changing the paradigm from 'race' to human genome variation. Nature Genetics, 36(11 Suppl), S5-7.

Ruff, C. C., Alexander, I. M., \& McKie, C. (2005). The use of focus group methodology in health disparities research. Nursing Outlook, 53(3), 134-140.

SAS Institute. (2004). $S A S^{\circledR}$ 9.1.3. Cary, N.C: SAS Institute.

Satcher, D., Pamies, R. J., \& Woelfl, N. N. (2006). Multicultural Medicine and Health Disparities: Essentials for Clinical Practice. New York: McGraw-Hill.

Schnall, E., Wassertheil-Smoller, S., Swencionis, C., Zemon, V., Tinker, L., O'Sullivan, M. J., et al. (2010). The relationship between religion and cardiovascular outcomes and all-cause mortality in the women's health initiative observational study. Psychology and Health, 25(2), 249-263.

Schlundt, D. G., Hargreaves, M. K., \& McClellan, L. (2006). Geographic clustering of obesity, diabetes, and hypertension in Nashville, Tennessee. The Journal of Ambulatory Care Management, 29(2), 125-132.

Schouten, B. C., \& Meeuwesen, L. (2006). Cultural differences in medical communication: A review of the literature. Patient Education and Counseling, 64(13), 21-34.

Schroeder, S. A. (2007). Shattuck lecture. We can do better-improving the health of the American people. The New England Journal of Medicine, 357(12), 1221-1228. 
Schwarz, M., Landis, S. E., Rowe, J. E., Janes, C. L., \& Pullman, N. (2000). Using focus groups to assess primary care patients' satisfaction. Evaluation and the Health Professions, 23(1), 58-71.

Seid, M., Stevens, G. D., \& Varni, J. W. (2003). Parents' perceptions of pediatric primary care quality: Effects of race/ethnicity, language, and access. Health Services Research, 38(4), 1009-1032.

Sempos, C. T., Bild, D. E., \& Manolio, T. A. (1999). Overview of the Jackson Heart Study: A study of cardiovascular diseases in African American men and women. The American Journal of the Medical Sciences, 317(3), 142-146.

Shaw, J. W., Johnson, J. A., Chen, S., Levin, J. R., \& Coons, S. J. (2007). Racial/ethnic differences in preferences for the EQ-5D health states: Results from the U.S. Valuation study. Journal of Clinical Epidemiology, 60(5), 479-490.

Sherman, J. E., Spencer, J., Preisser, J. S., Gesler, W. M., \& Arcury, T. A. (2005). A suite of methods for representing activity space in a healthcare accessibility study. International Journal of Health Geographics, 4, 24.

Shwartz, M., Ash, A. S., Anderson, J., Iezzoni, L. I., Payne, S. M., \& Restuccia, J. D. (1994). Small area variations in hospitalization rates: How much you see depends on how you look. Medical Care, 32(3), 189-201.

Siminoff, L. A., Graham, G. C., \& Gordon, N. H. (2006). Cancer communication patterns and the influence of patient characteristics: Disparities in information-giving and affective behaviors. Patient Education and Counseling, 62(3), 355-360.

Smedley, B. D., Stith, A. Y., \& Nelson, A. R. (2003). Unequal Treatment: Confronting Racial and Ethnic Disparities in Health Care. Washington D.C.: National Academy Press.

Smith, A. K., Davis, R. B., \& Krakauer, E. L. (2007). Differences in the quality of the patient-physician relationship among terminally ill African-American and white patients: Impact on advance care planning and treatment preferences. Journal of General Internal Medicine, 22(11), 1579-1582.

Soh, N. L., Touyz, S. W., \& Surgenor, L. J. (2006). Eating and body image disturbances across cultures: A review. European Eating Disorders Review, 14(1), 54-65.

Souchek, J., Byrne, M. M., Kelly, P. A., O' Malley, K., Richardson, M., Pak, C., et al. (2005). Valuation of arthritis health states across ethnic groups and between patients and community members. Medical Care, 43(9), 921-928.

SPSS Inc. (2009). PASW Statistics 18. Chicago, Ill.: SPSS. 
Stewart, A. L., \& Nápoles-Springer, A. M. (2003). Advancing health disparities research: Can we afford to ignore measurement issues? Medical Care, 41(11), 1207-1220.

Takakuwa, K. M., Shofer, F. S., Limkakeng Jr, A. T., \& Hollander, J. E. (2008). Preferences for cardiac tests and procedures may partially explain sex but not race disparities. The American Journal of Emergency Medicine, 26(5), 545-550.

Taylor, H. A., Wilson, J. G., Jones, D. W., Sarpong, D. F., Srinivasan, A., Garrison, R. J., Wyatt, S. B. (2005). Toward resolution of cardiovascular health disparities in African Americans: Design and methods of the Jackson Heart Study. Ethnicity and Disease, 15(4 Suppl 6), S6-4-17.

Thrasher, A. D., Earp, J. A., Golin, C. E., \& Zimmer, C. R. (2008). Discrimination, distrust, and racial/ethnic disparities in antiretroviral therapy adherence among a national sample of hiv-infected patients. Journal of Acquired Immune Deficiency Syndromes, 49(1), 84-93.

Thomas, D. C. (2004). Statistical Methods in Genetic Epidemiology. New York: Oxford University Press.

Toldson, I. L., \& Toldson, I. A. (2001). Biomedical ethics: An African-centered psychological perspective. Journal of Black Psychology, 27(4), 401-423.

Tyson, S. Y. (2007). Can cultural competence be achieved without attending to racism? Issues in Mental Health Nursing, 28(12), 1341-1344.

Voelker, R. (2008). Decades of work to reduce disparities in health care produce limited success. JAMA: The Journal of the American Medical Association ，299(12), 14111413.

Washington, H. A. (2006). Medical Apartheid: The Da rk History of Medical Experimentation on Black Americans from Colonial Times to the Present. New York: Random House, Inc.

Washington, H. A., Baker, R. B., Olakanmi, O., Savitt, T. L., Jacobs, E. A., Hoover, E.,et al. (2009). Segregation, civil rights, and health disparities: The legacy of African American physicians and organized medicine, 1910-1968. Journal of the National Medical Association, 101(6), 513-527.

Watson, W. (1998). Black Folk Medicine: The Therape utic Significance of Faith and Trust. New Brunswick: Transaction Publishers.

White-Means, S. I. (1995). Conceptualizing race in economic models of medical utilization: A case study of community-based elders and the emergency room. Health Services Research, 30(1 Pt 2), 207-223. 
Wilkinson, D. Y., \& King, G. (1987). Conceptual and methodological issues in the use of race as a variable: Policy implications. The Milbank Quarterly, 65(Suppl 1), 56-71.

Wilkinson, R. G. (2001). Mind the Gap: Hierarchies, Health and Human Evolution. New Haven: Yale University Press.

Williams, D. R. (1997). Race and health: Basic questions, emerging directions. Annals of Epidemiology, 7(5), 322-333.

Williams, D. R., Costa, M. V., Odunlami, A. O., \& Mohammed, S. A. (2008). Moving upstream: How interventions that address the social determinants of health can improve health and reduce disparities. Journal of Public Health Management and Practice, 14(Suppl), S8-17.

Wilson, D. K., Williams, Z. L., Arheart, K., Bryant, E. S., \& Alpert, B. S. (1994). Race and sex differences in health locus of control beliefs and cardiovascular reactivity. Journal of Pediatric Psychology, 19(6), 769-776.

Wittenberg, E., Halpern, E., Divi, N., Prosser, L. A., Araki, S. S., \& Weeks, J. C. (2006). The effect of age, race and gender on preference scores for hypothetical health states. Quality of Life Res earch: An In ternational Journal of Quality of Life Aspects o $\quad f$ Treatment, Care and Rehabilitation. 15(4), 645-653. 
APPENDIX: JACKSON HEART STUDY CENSUS TRACTS

The Study Area for the Jackson Heart Study

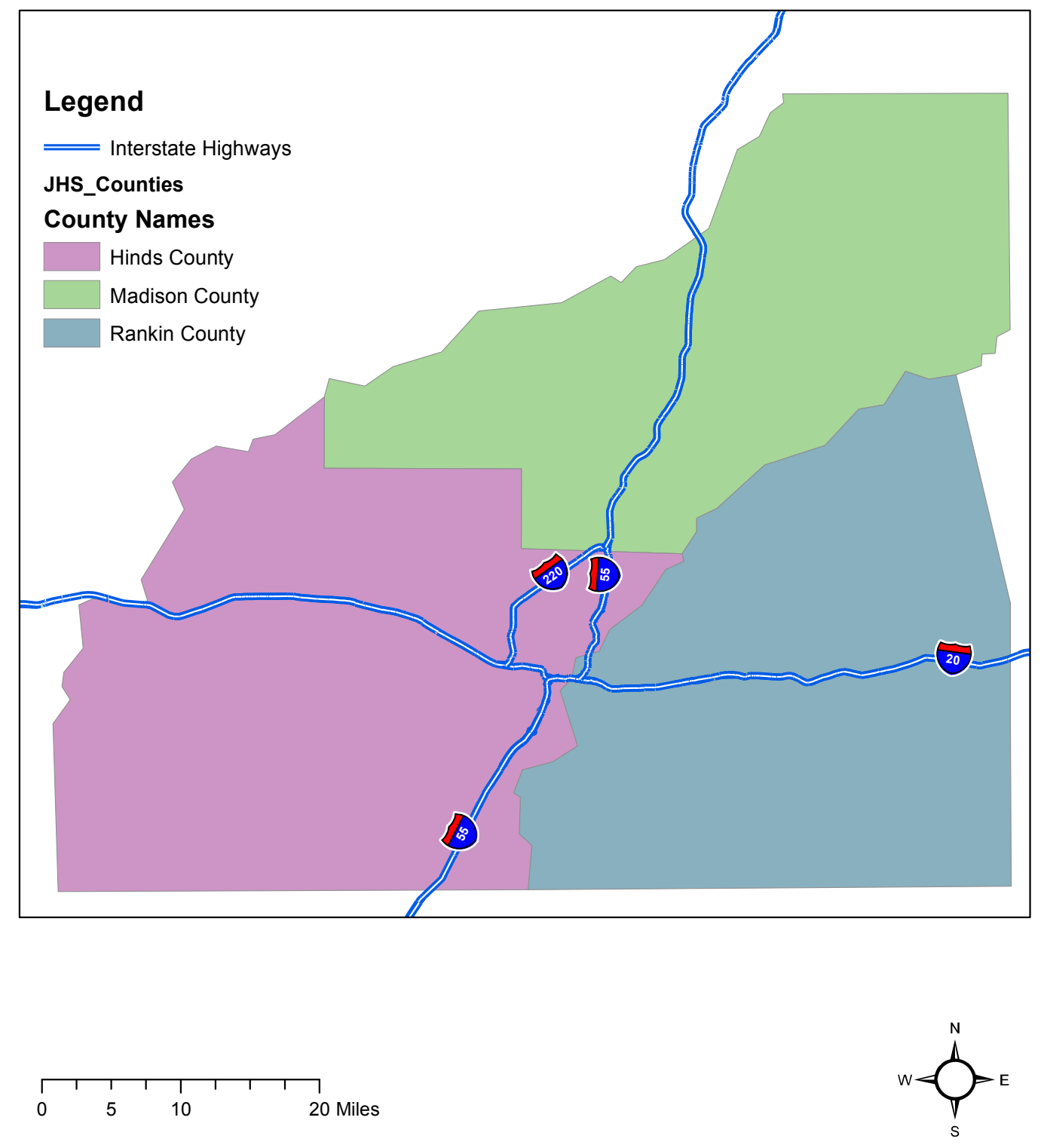




\section{JHS Percentage of Bachelors Degree by Census Tract}
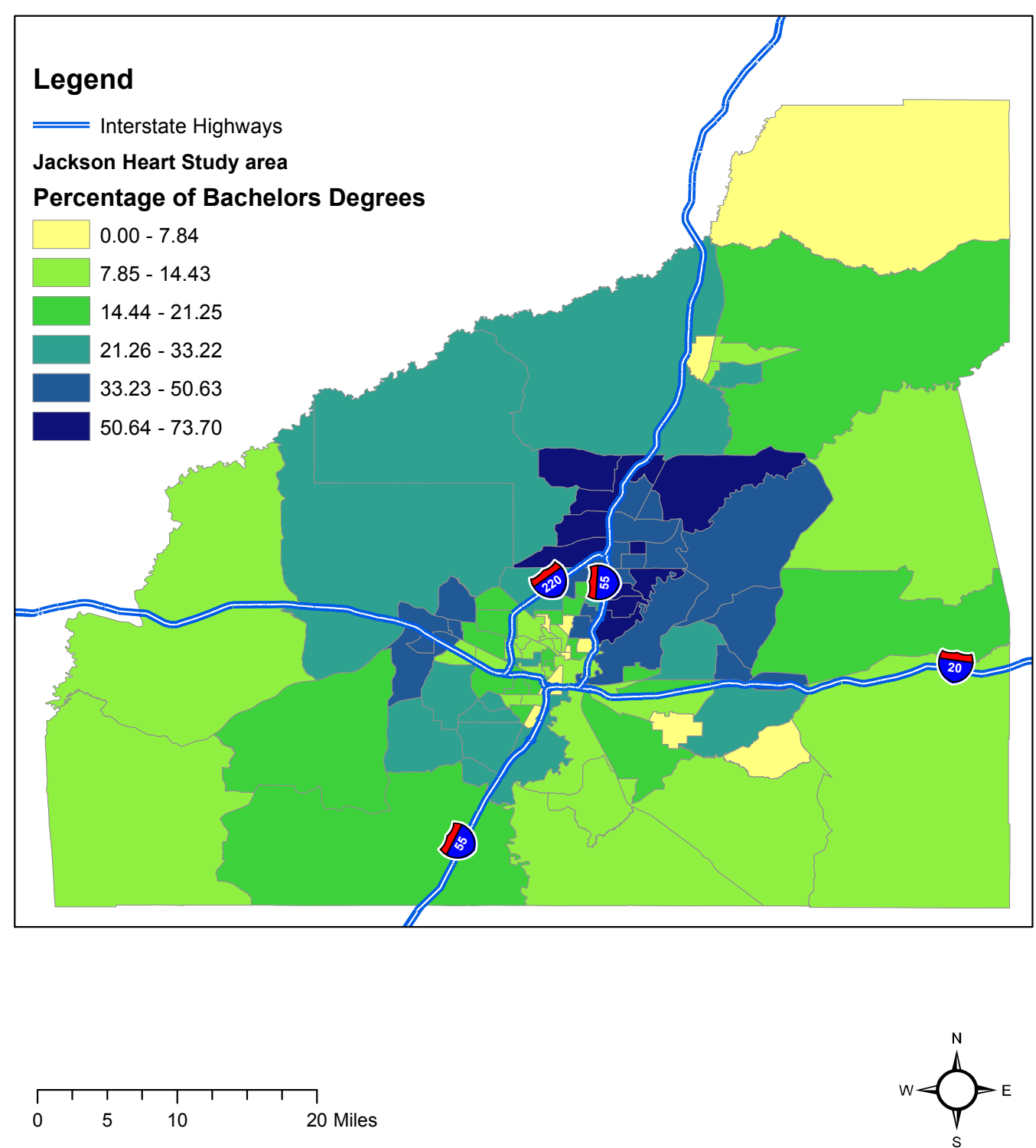
JHS Percentage with High School Diploma by Census Tract
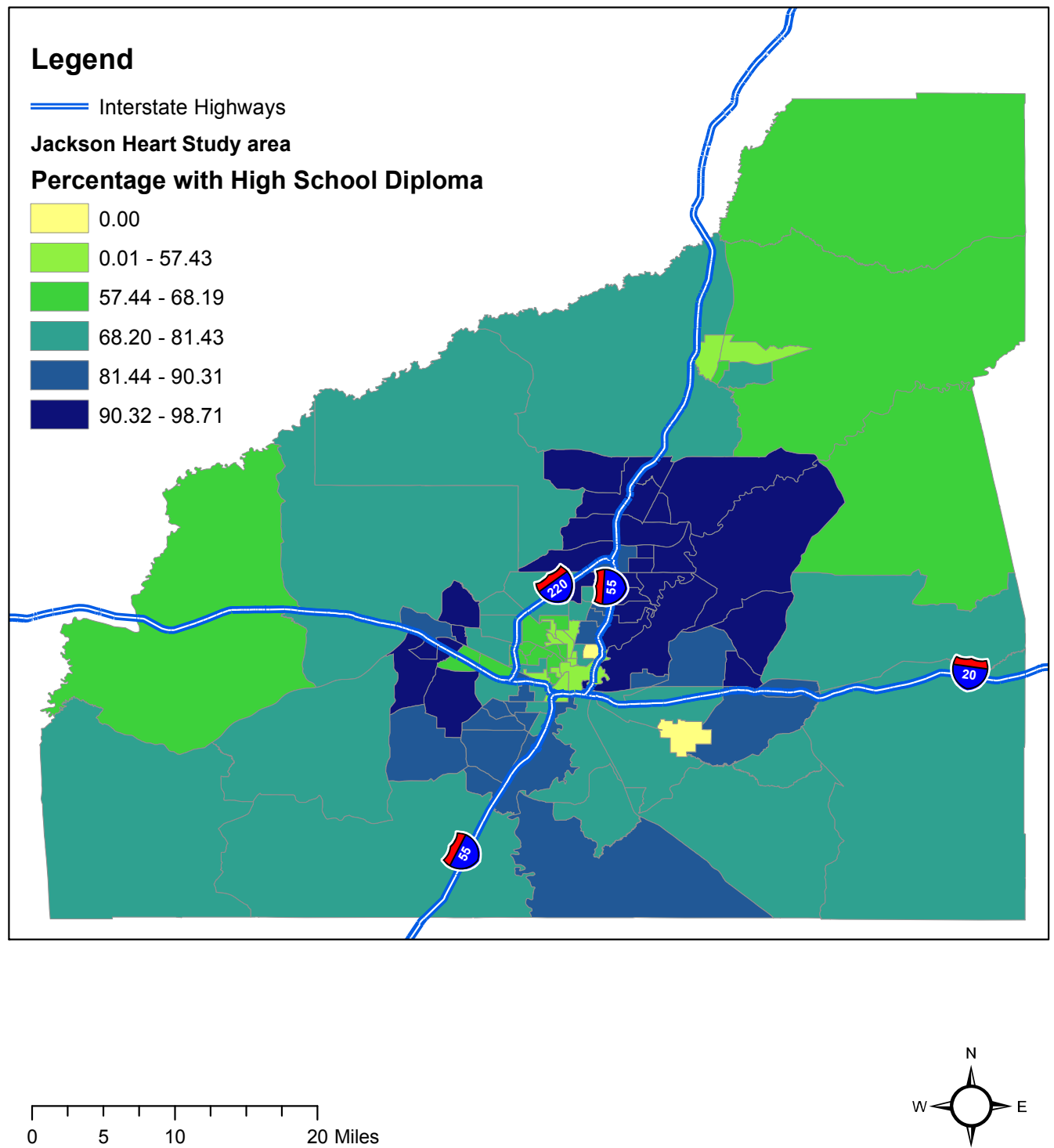

$=\overbrace{S}^{N}=$ 
JHS Median Annual Household Income by Census Tract
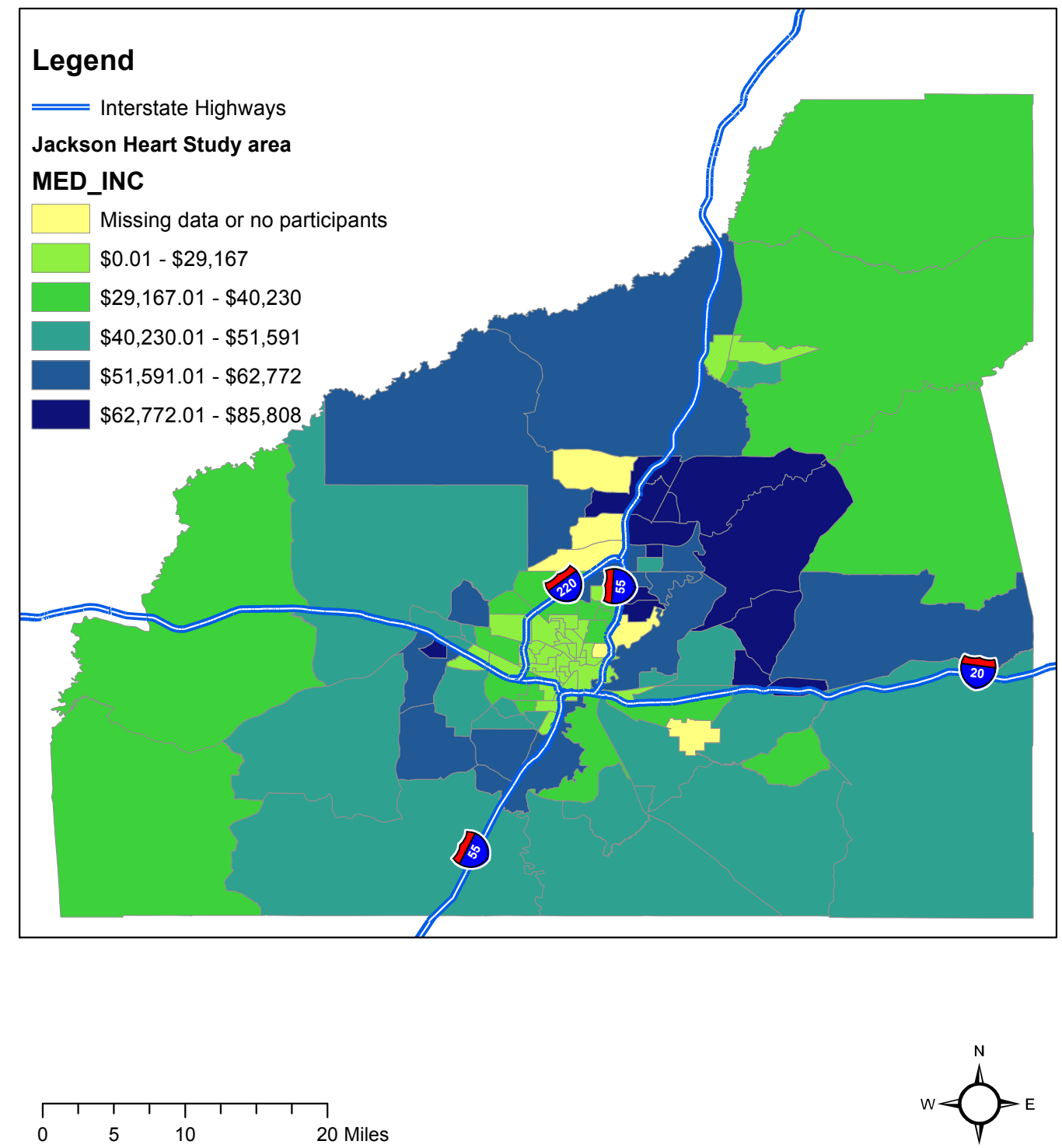

- pos 
JHS Percentage of African Americans by Census Tract
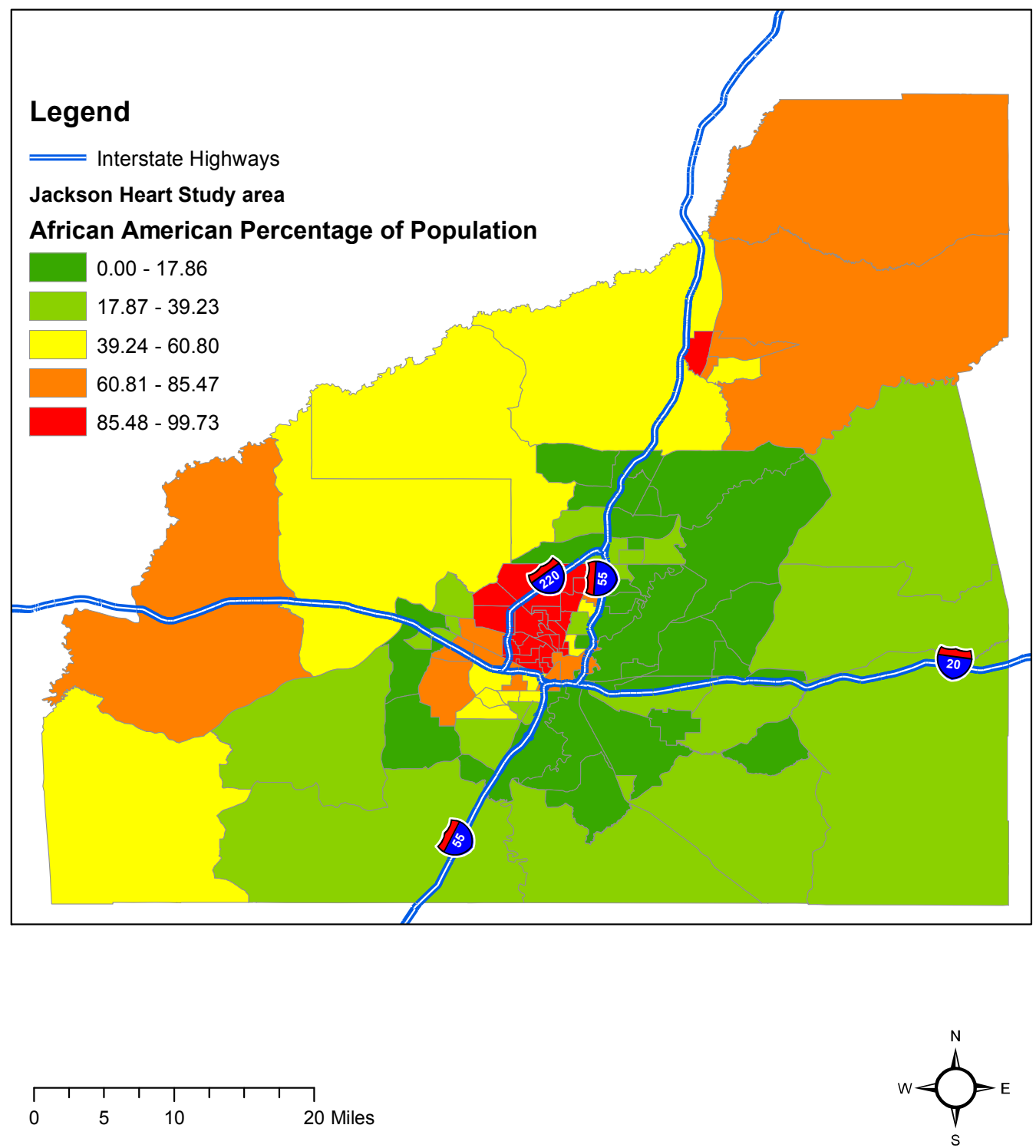


\section{JHS Least Advantaged Census Tracts}

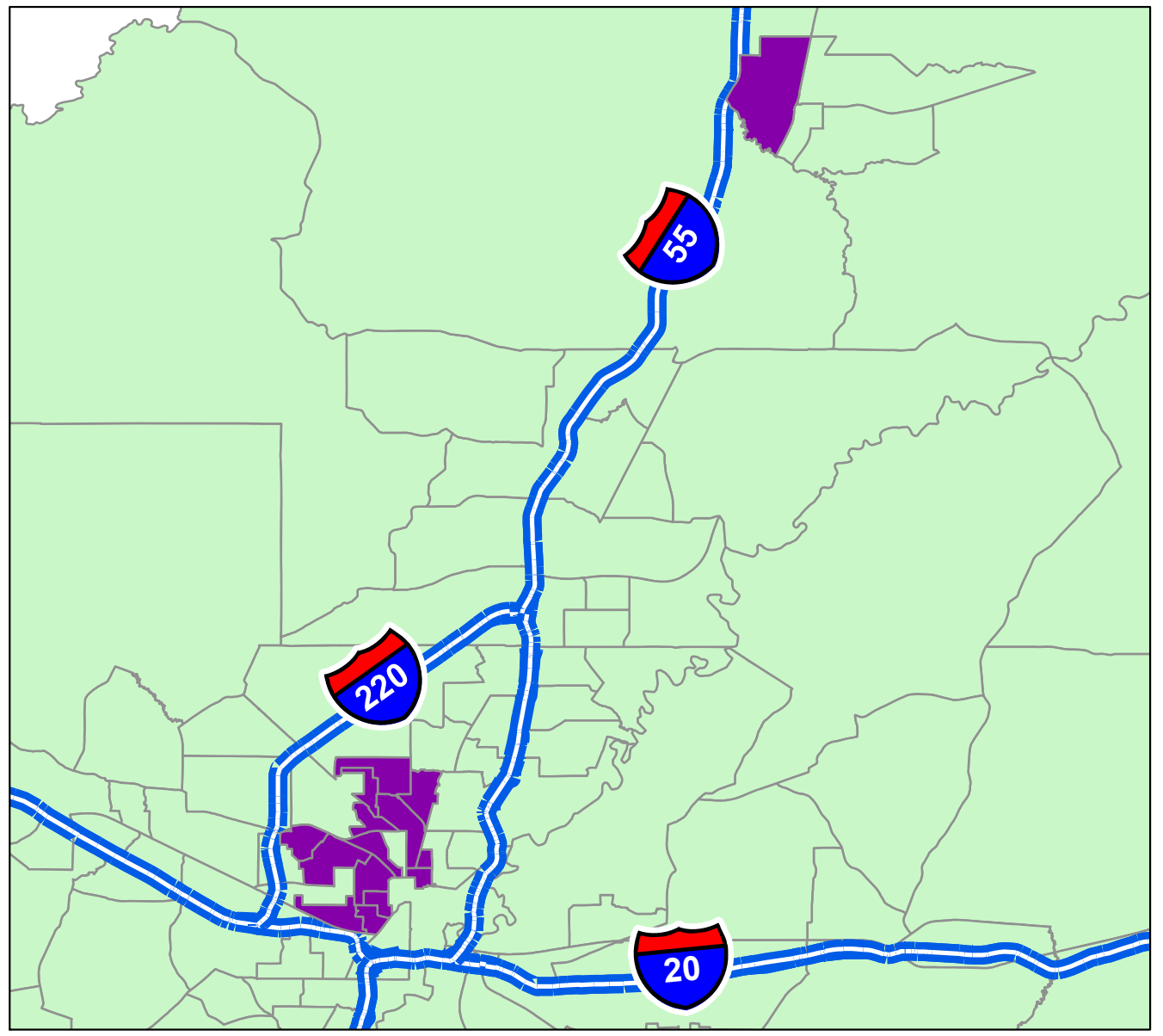

\section{Legend}

Jackson Heart Study area selection

Interstate Highways

Jackson Heart Study area

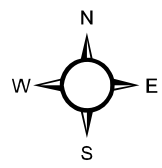




\section{VITA}

Lawrence Tarves Brown was born to Lawrence Robert Brown and Sharon Eressie Brown in 1978. He was reared in his hometown, West Memphis, Arkansas, before his family moved to League City, Texas-where he graduated from Clear Creek High School in May 1997. Lawrence proceeded to attend the prestigious Morehouse College in Atlanta, Georgia, and graduated magna cum laude with a degree in African American Studies in May 2001. During his tenure at Morehouse College, Lawrence was active in community projects dealing with community revitalization and started an afterschool program for Atlanta Housing Authority children.

Because of his interest in the role of government in affecting communities, Lawrence enrolled in the University of Houston's Master of Public Administration program in 2004. After graduating from the University of Houston in June 2006, Lawrence began work on his doctorate in Health Science Administration-which later became Health Outcomes and Policy Research — at the University of Tennessee Health Science Center. While working on his doctorate, Lawrence worked as the Student Research Coordinator on the research project entitled Partnership for Asthma Trigger-Free Homes (PATH) led by LeMoyne Owen College. The primary investigator for PATH was Dr. Cheryl Golden. Lawrence also played a pivotal role in assisting Dr. Shelley White-Means in the founding of the Consortium for Health Education, Economic Empowerment, and Research (CHEER), a NIH-funded exploratory health disparities center. His research on health disparities was presented at the University of Tennessee Health Science Center in August 2010. 